\title{
THE SURGICAL JOURNEY: EXPLORING THE OR-PACU HANDOVER
}

\author{
By \\ Marketa Gross \\ BScN, 2014 \\ Ryerson University, Toronto, Canada,
}

\author{
A thesis \\ presented to Ryerson University \\ in partial fulfillment of the \\ requirements for the degree of \\ Master of Nursing \\ In the Program of
}

Nursing

Toronto, Ontario, Canada, 2017

(C) Marketa Gross 2017 


\section{AUTHOR'S DECLARATION}

I hereby declare that I am the sole author of this thesis. This is a true copy of the thesis, including any required final revisions, as accepted by my examiners.

I authorize Ryerson University to lend this thesis to other institutions or individuals for the purpose of scholarly research.

I further authorize Ryerson University to reproduce this thesis by photocopying or by other means, in total or in part, at the request of other institutions or individuals for the purpose of scholarly research.

I understand that my thesis may be made electronically available to the public. 


\title{
The Surgical Journey: Exploring the OR-PACU Handover
}

\author{
Marketa Gross \\ Master of Nursing, 2017 \\ Master of Nursing Degree Program \\ Daphne Cockwell School of Nursing, Ryerson University, Toronto
}

\begin{abstract}
Patient safety in health care remains a serious concern in Canada. Adverse events can lead to physiological and psychological complications and pose a significant economic burden on the health care system. The purpose of this descriptive qualitative study was to explore the team processes, roles and factors that underpin effective communication between team members during an OR-PACU handover. Content analysis revealed four major categories: Ownership, Distractions and Interruptions, Transfer of Information and Workflow. The results of this study, informed by the Theory of Collective Competence enhance our understanding of the OR-PACU handover and support the need for the development of a structured OR-PACU team handover process.
\end{abstract}

Keywords: OR-PACU handover, handoff, transfer of care, postoperative period, perianaesthesia care unit, recovery room, perioperative communication, interprofessional communication, interprofessional collaboration, collective competence 


\section{Acknowledgements}

I would like to express my deep appreciation and thanks to my thesis supervisor Dr.

Sherry Espin. Her knowledge and expertise helped me to keep focused and her never ending enthusiasm and continuous support kept me going. She is an inspiration to many and I would like to thank her for encouraging me.

I would like to acknowledge my thesis committee members Dr. Karen LeGrow, Rachel Grant and Dr. Zeev Friedman. In a unique way, they all provided new perspectives and valuable suggestions throughout the study. Their expertise and insight into the field of communication and interprofessional collaboration are much appreciated and provided notable contributions to this process.

I would like to acknowledge the participants in this study: Thank you all for being part of this study and for sharing your time and experiences with me.

My dear colleagues and friends: Thank you for your support and understanding. Kristen Cunningham, Claudia Williams, and Antoniette Labricciosa you have been endless support, wonderful mentors and role models. Thank you for keeping me sane throughout this process.

To my family, to my dear husband Allan Gross and my children, Alani and Shaye: Thank you is not enough. I wish to express my deepest gratitude for the patience and tolerance you provided to me during this process, for not letting me quit and for believing that we can do this. I love you guys. 


\section{Dedications}

This thesis is dedicated to my husband Allan Gross without whom this work would not be possible. Thank you for your guidance, for listening, for keeping our family going, and for being there for me, always.

ILY mtef mdm and more 


\section{Table of Contents}

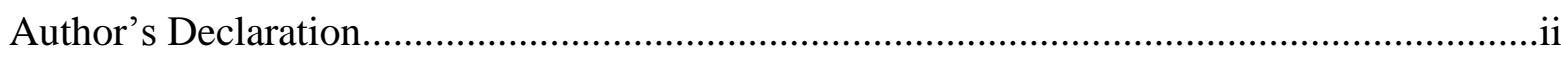

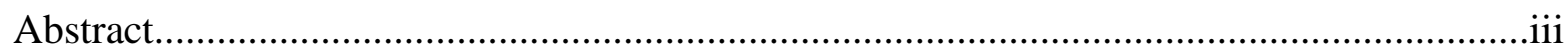

Acknowledgement ............................................................................................................

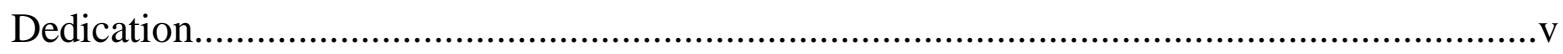

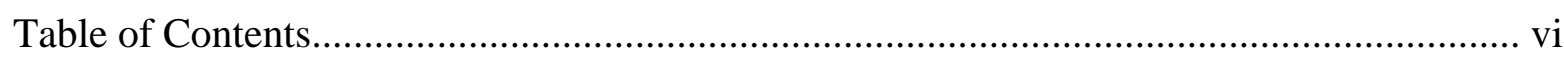

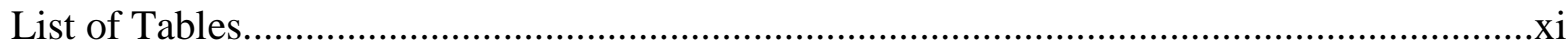

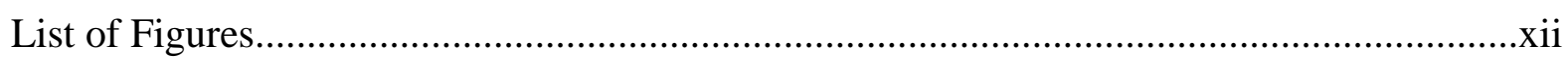

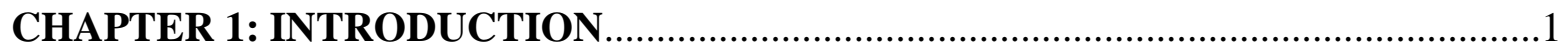

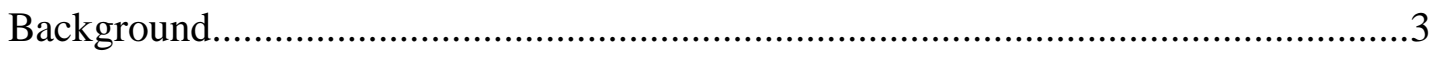

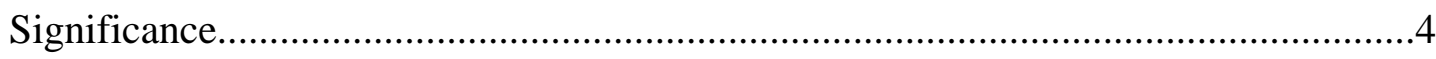

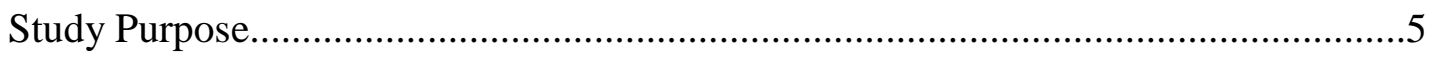

CHAPTER 2: REVIEW OF THE LITERATURE..................................................

Theoretical Framework...................................................................................6

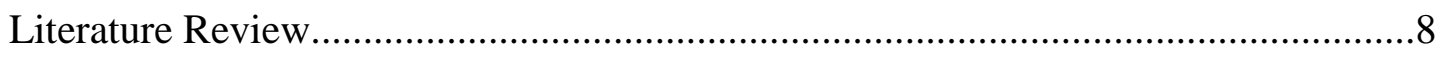

Search Strategy

Conceptual Definition: Handover..............................................................

Interprofessional Communication and Teamwork...........................................10

Factors Influencing Handover......................................................................13

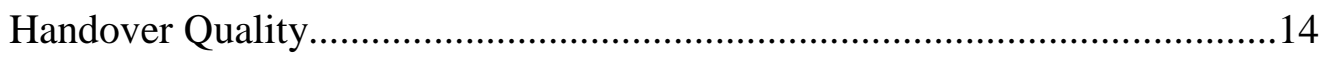

Standardised Handover Process.....................................................................16

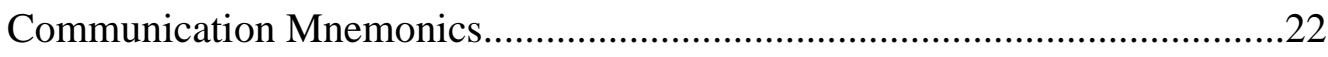

Compliance with Standardized Handover Process...........................................25

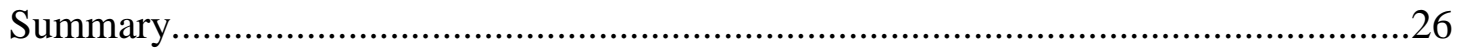




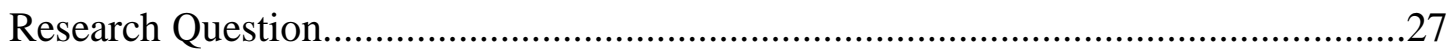

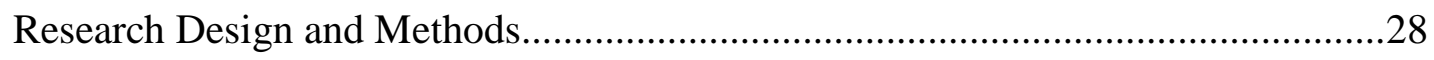

Research Paradigm.............................................................................................29

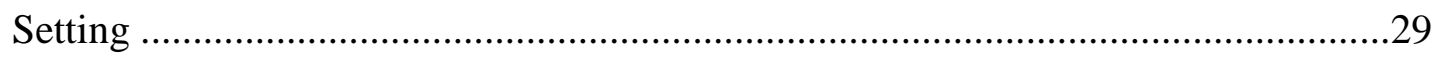

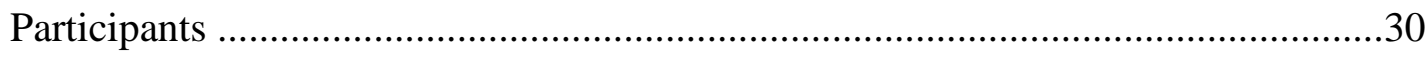

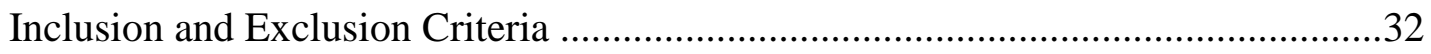

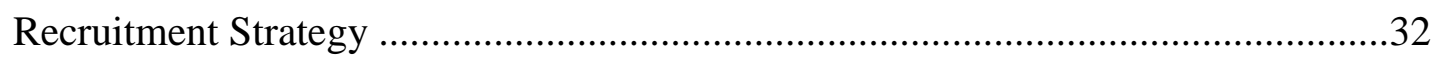

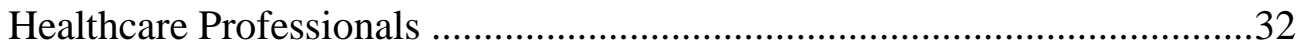

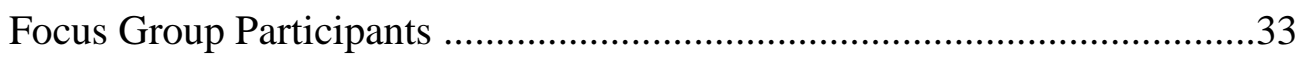

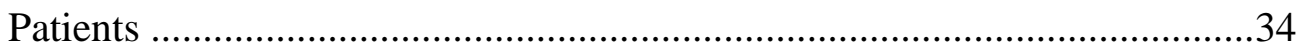

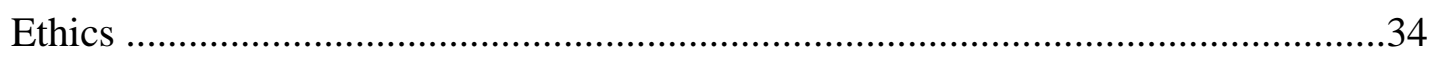

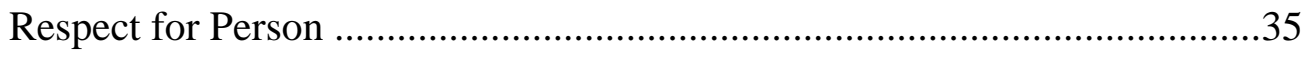

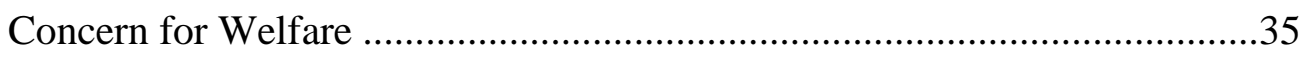

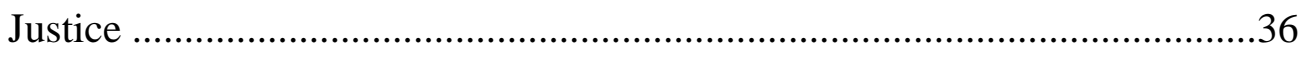

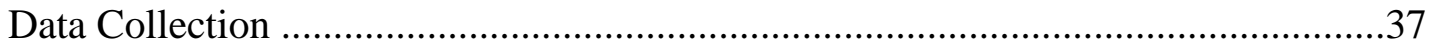

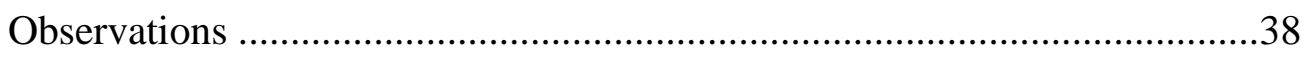

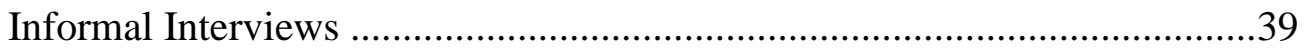

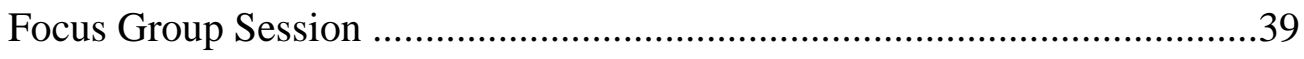

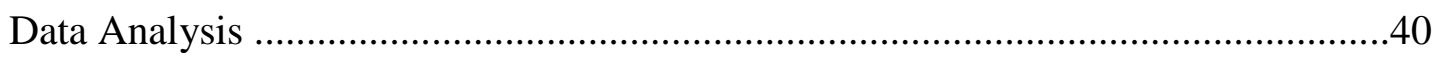

Phase 1: Preparation ...............................................................................42

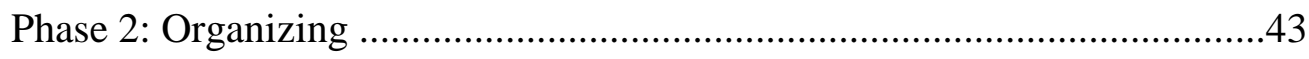

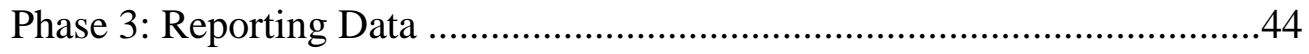




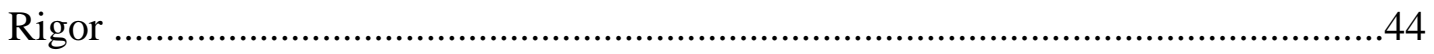

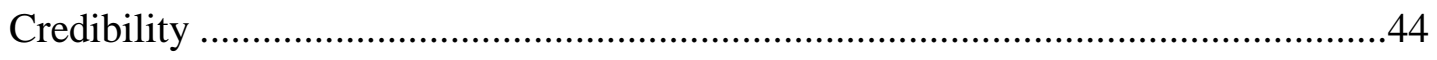

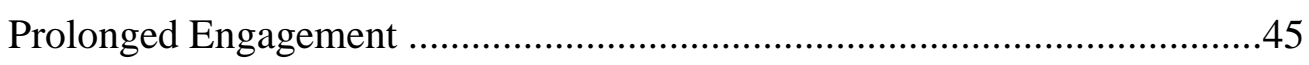

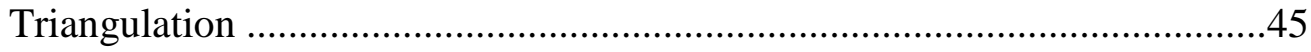

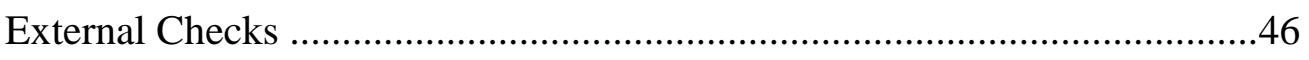

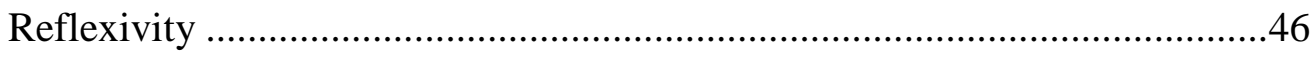

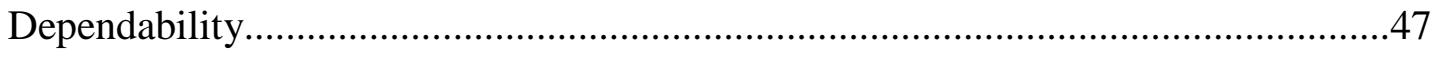

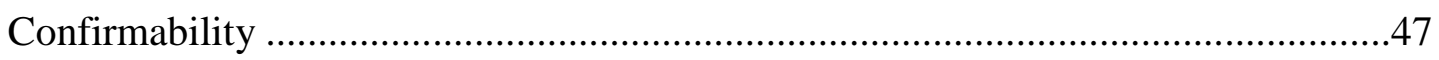

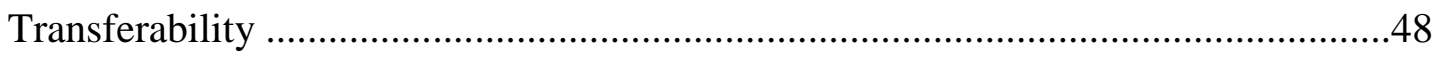

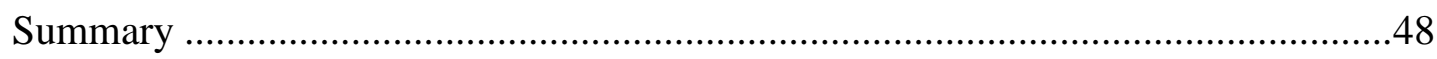

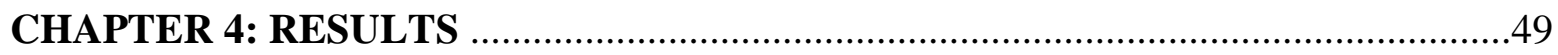

Descriptive information: Participants .........................................49

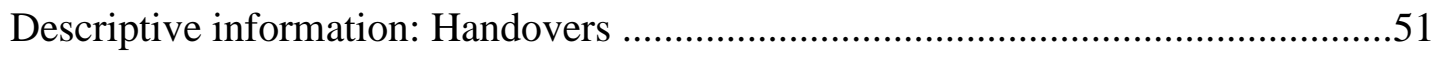

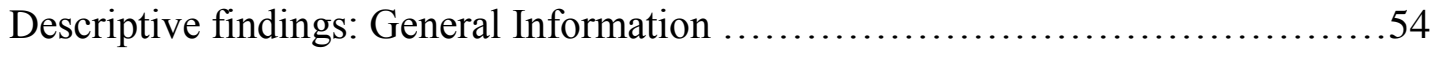

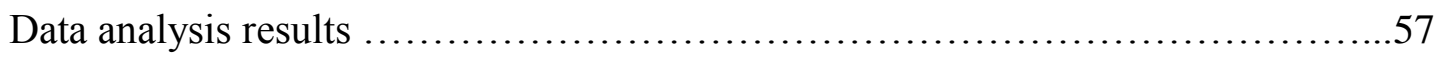

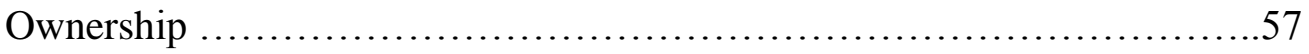

Distractions and Interruptions .....................................61

Transfer of Information ............................................ 67

Workflow ......................................................... 75

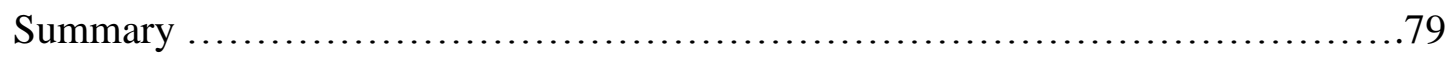

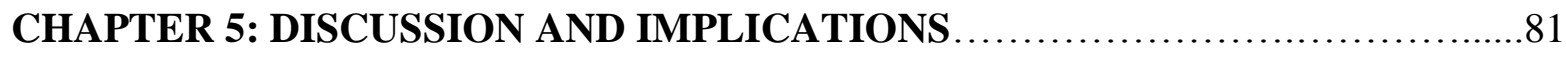

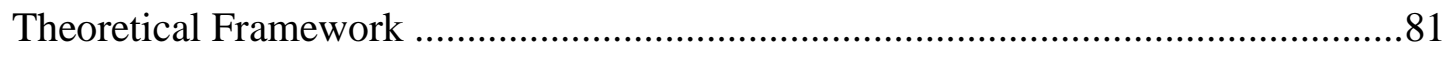

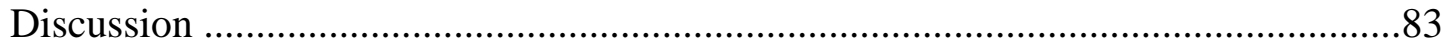




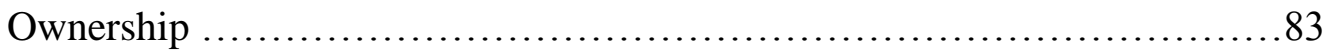

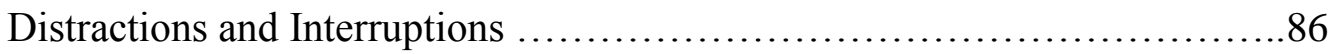

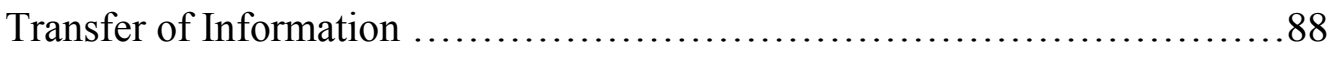

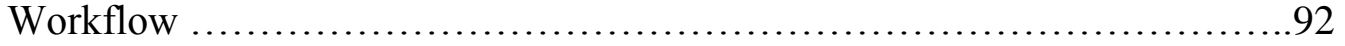

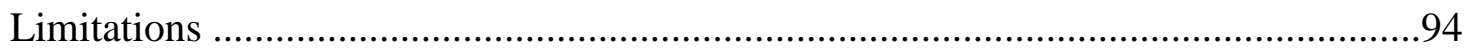

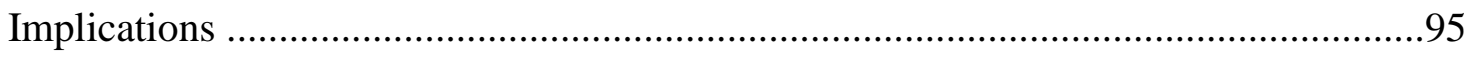

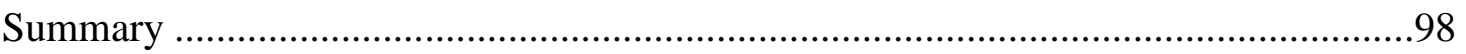

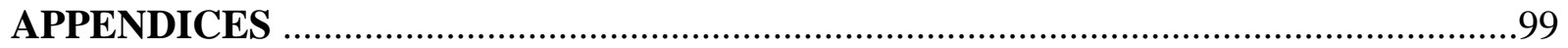

Appendix A. Recruitment Notice Patients .......................................................99

Appendix B. Recruitment Notice Healthcare Professionals .....................................100

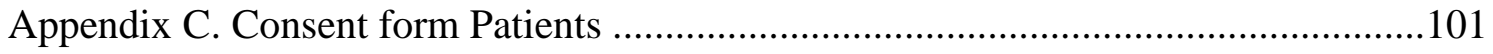

Appendix D. Consent Form Healthcare Professionals ...........................................107

Appendix E. Consent Form Healthcare Professionals- Focus Group .........................113

Appendix F. Consent Form Patients- Additional Verbal ...........................................119

Appendix G. Consent Form Healthcare Professionals- Additional Verbal ..................120

Appendix H. Sample Questions Patients ................................................................121

Appendix I. Sample Questions Healthcare Professionals ......................................122

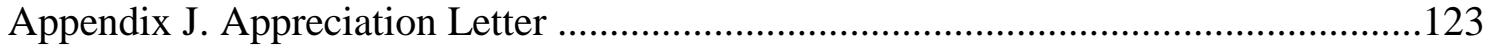

Appendix K. Appreciation Letter- Focus Group Participants ..................................124 


\section{REFERENCES}




\section{LIST OF TABLES}

Table

Page

1. Literature assessing the impact of an implementation, adaptation and evaluation of a postoperative handover protocol......................................................... 16

2. Frequently used mnemonics during handovers.............................................. 24

3. Examples of Data Analysis Process: Unit of Analysis, Meaning Unit, Condensed Meaning Unit, Code, Subcategory and Category $\ldots \ldots \ldots \ldots \ldots \ldots \ldots \ldots \ldots \ldots \ldots . \ldots \ldots$

4. a) OR-PACU Handover Study: Participants Information: Patients...................... 49

b) OR-PACU Handover Study: HCP Participants Information........................... 50

c) OR-PACU Handover Study: Recruitment Information Focus Group Session.... 51

5. a) Descriptive Information on the OR-PACU Handovers in this study................. 52

b) Handover Type by Patient Admission Status.............................................. 52

c) Handover Type by Surgical Specialty Type.............................................. 53

d) Handover Timing Information............................................................... 54

6. a) Handover Participation Information for Anaesthesia Providers...................... 55

b) Handover Participation Information for Surgical Providers........................... 55

c) Handover Participation Information for Registered Nurses: OR and PACU..... $\quad 55$

7. PACU Nurses Availability and Privacy Considerations................................... 56 


\section{LIST OF FIGURES}

Figures $\quad$ Page

1. Coding Tree: Exploring OR-PACU Handovers Study: Data Analysis Results..... 57

a) Exploring OR-PACU Handovers: Main Category: Ownership............... 58

b) Exploring OR-PACU Handovers: Main Category: Distractions and

Interruptions................................................. 62

c) Exploring OR-PACU Handovers: Main Category: Transfer of Information..... 67

d) Exploring OR-PACU Handovers: Main Category: Workflow............... 75 


\section{CHAPTER 1: INTRODUCTION AND BACKGROUND}

Operating Room (OR) - PeriAnaesthetic Care Unit (PACU) handovers are often inaccurate and incomplete affecting patient safety and placing the surgical patient at risk for

adverse events (Milby, Bohmer, Gerbershagen, Joppich, \& Wappler, 2014; Nagpal et al., 2013).

There are many factors affecting patient safety that lead to adverse events and ineffective communication or communication failures which are classified as incomplete, inaccurate or fragmented information transfers at any point of care and are the leading cause of preventable adverse events in health care (Nagpal et al., 2013; Halverson et. al., 2011). Furthermore, ineffective communication during transfer of care points, such as the surgical handover, accounts for at least half of these preventable adverse events.

Adverse events can be defined as unintentional harm or complications not related to the underlying disease and can lead to a prolonged hospital stay, disability, or even death (Baker et al., 2004; Canadian Patient Safety Institute (CPSI), 2011). The Joint Commission on Accreditation of Healthcare Organizations (JCAHO) identified communication between health care providers (HCPs) and continuum of care and planning as the top ten root causes for adverse events in the post-operative complications category (JCAHO, 2015). Patient safety in health care remains a serious concern in Canada. Between 9,000 and 24,000 Canadians die annually following a preventable adverse event (Baker et al., 2004). Furthermore, adverse events contribute to an increased financial burden on the healthcare system. Recent data show that the economic burden associated with adverse events in Canada per year is estimated at 1.1 billion dollars, out of which $\$ 397$ million dollars is related to preventable adverse events (CPSI), 2011).

Handovers can be defined as a transfer of care, responsibility and accountability for a patient on a permanent or a temporary basis and require clear and accurate communication and 
effective teamwork (Nagpal et al., 2013). OR-PACU handovers can be further defined as the transfer of care, responsibility and accountability for the surgical patient coming from the OR to the PACU in the immediate postoperative period. Surgical patients often experience multiple transfer of care points throughout their surgical journey. A typical journey for a patient scheduled for an elective procedure includes a visit to the physician's office, the surgeon's office, the pre-admit department, the admitting department, the pre-operative check in, the operating room, the post-operative destination, the surgical floor, and home or a rehabilitation facility. Each of these destinations represents a transfer of care point in which there are often new health care providers introduced to the patient. Increased number of transfer of care points and handovers increases the chances of communication errors, as such effective and accurate communication is essential in providing safe care (Petrovic et al., 2015).

The destination for the surgical patient following the completion of the surgical procedure is dependent on overall health status and acuity. Typically the most common destination for the surgical patient in the immediate postoperative period is the periAnaesthetic care unit (PACU) or the intensive care unit (ICU). The patient is most commonly accompanied during the transfer from the OR to PACU by the anaesthesia provider, the perioperative nurse and occasionally the surgical provider. The surgical patient is especially vulnerable in the immediate postoperative period due to the effects of the anaesthesia and surgery. Complicating this is the time during ORPACU handovers can be very chaotic (Napgal et al., 2011). The PACU nurse focuses on stabilizing and assessing the surgical patient while the other handover participants (anaesthesia provider, perioperative nurse and surgical provider) are all competing for attention and who will complete the handover report first. Surgical handovers are often complex, interprofessional and hierarchal and evidence also suggests that OR-PACU handovers are faced with many distractions 
all of which can impact the surgical patient's safety (Milby et al., 2014; Petrovic et al., 2012;

Siddiqui et al., 2012).

\section{Background:}

There has been a growing interest in improving OR-PACU handovers and evidence shows that interventions directed towards improving handovers enhance teamwork, team effectiveness and efficiency (Siddiqui et al., 2012; Segall et al., 2012). There is a large body of research exploring communication, effective teamwork and interprofessional collaboration within the perioperative environment to enhance safe and efficient care to the surgical patient (Huang, Kim, \& Berry, 2013; Healey, Undre, Sevdalis, Koutantji, \& Vincent, 2006; Lingard, Reznick, Espin, Regher, \& DeVito, 2002; Lingard, Regehr, Espin, \& Whyte, 2006; Lingard et al., 2008; Smith \& Jones, 2009). There are also initiatives that have been implemented within the operating room to improve patient safety by standardizing the communication process, such as the World Health Organization (WHO) Surgical Safety Checklist (2009). The checklist supports the notion that patient safety is not a solitary responsibility but rather relies on wellfunctioning interprofessional collaboration in which all members of the perioperative team are responsible (WHO, 2009). Similarly, effective postoperative handovers require collaboration and participation of all teams involved in the care of the patient (Manias, Geddes, Watson, Jones \& Della, 2015; Segall et al., 2012). Several studies have demonstrated a significant reduction in task errors and incomplete information transfer with a standardized OR-PACU handover process (Milby et al., 2014; Napgal et al., 2013; Boat \& Spaeth., 2013). However, existing literature also suggests that OR-PACU handovers often lack structure and involvement from all members of the interprofessional team which leads to an overall incomplete or inconsistent transfer of information (Segall et al, 2012; Siddiqui et al., 2012; Milby et al., 2014; Napgal et al., 2011). 
Studies exploring OR-PACU handovers are focused on assessing the handover from the perspective of the individual discipline, such as nursing, anaesthesia, or surgical teams and provide limited understanding of the interprofessional roles and dynamic during handovers (Bourdon, 2015; Manias et al., 2015; Milby et al., 2014; Segall et al., 2012). There currently exists only a few OR-PACU handover tools that have been designed, implemented, and successfully tested (Manser, Foster, Gisin, Jaeckel, \& Ummenhofer, 2010; Napgal et al., 2013; Saltzvel et al., 2013; Siddiqui et al., 2012). These tools focus mainly on the role of the anaesthesia provider. Minimal data is available for a handover tool that would include the anaesthesia provider, OR nurse and a member of the surgical team working in collaboration during OR-PACU handovers. As such, currently existing OR-PACU handover tools are encouraging professionals to work in silos rather than interprofessionally. In addition, findings from studies also show that most current standardized handover tools are focused purely on information transfer, are point and check mark oriented and overall lack structure that can improve and encourage interprofessional communication (Bourdon, 2015; Manias et al., 2015; Manser et al., 2010; Milby et al., 2014).

\section{Significance:}

Existing research assessing the handover process and interventions is mainly focused on information transfer and does not provide insight into the factors and dynamics which prevent the handover from being a team oriented process or how it is perceived by the various team members (Randmaa, Martensson, Swenne, \& Engstrom, 2015; Segall et al., 2012). There are no studies available that discuss the development of a team OR-PACU handover tool based on contextual factors that affect the handover and incorporate collaboration of the anaesthesia provider, the PACU nurse, the perioperative nurse and a member of the surgical team. 
Understanding the role of individual team members and existing handover practices could provide insight into developing strategies towards more effective OR-PACU handovers.

Furthermore, there is a need to develop an OR-PACU team oriented handover tool that will not only function as a guide to information transfer but will also lead and structure the communication process.

\section{Study purpose}

The purpose of this descriptive qualitative study was to explore the team

processes, roles, and factors that underpin effective communication between team members during an OR-PACU handover. 


\section{CHAPTER 2: LITERATURE REVIEW}

The following chapter will provide an overview of the theoretical framework which informed the results of this study the Theory of Collective Competence (Boreham, 2004) followed by an overview of the existing literature related to OR-PACU handovers. The literature review is divided into the following sections: 1) interprofessional communication and teamwork, 2) factors influencing handover, 3) handover quality 4) standardized handover process, 5) communication mnemonics, and 6) compliance with standardized handover process.

\section{Theoretical Framework}

\section{Theory of Collective Competence}

Qualitative descriptive research is situated in the naturalistic inquiry and there is no prior commitment to a particular theoretical or philosophical framework (Sandelowski, 2000). The methodology allows the researcher to represent the data in an informed and natural state not bound by theory but rather to choose a theory that can enhance comprehensive understanding of the studied phenomenon (Sandelowski, 2000; Sandelowski, 2010). The Theory of Collective Competence closely relates to interprofessional collaboration within the health care setting and provided the theoretical lens to the interpretation of the results of this study (Boreham, 2004; Hodges \& Lingard, 2012).

Collective competence is based on how individuals function in a team to create collective decisions and actions in the workplace (Boreham, 2004). Collectivists reduce their own needs to the group priorities, harmony and activities rather than individual gratification (Boreham, 2004). The concept of collective competence is rooted in learning and activity theory and consists of 
three parts: 1) making collective sense of events in the workplace, 2) developing and using a collective knowledge base, and 3) developing a sense of interdependency (Boreham, 2004).

1) Making collective sense of events in the workplace is the initial step for a group to function competently. The first step towards collective competence is for the group to have the capacity to understand the situation the group is presented with and consideration of language that plays a central role in making sense of the situation in a narrative format (Boreham, 2004).

2) Developing and using a collective knowledge base is the second step during which the individuals in the group make collective sense of events. This step is directly dependent on the collective knowledge base of the team (Boreham, 2004). Collective knowledge is often dependent on the group members' interaction and develops as a result of interpretations of common experiences and a mutual agreement on the situation (Boreham, 2004).

3) Developing a sense of interdependency within the group is the third requirement of collective competence. This step is based on collaboration and communication between the team-members and the ability to overcome fragmentation and individual differences for the team to function competently (Boreham, 2004).

The concept of collective competence has been widely accepted in the aviation industry where creating high functioning and competent teams is a priority (Boreham, 2004). Collective competence has also been adopted in medical education and has gained interest in other disciplines, such as management and finance, engineering and music (Hodges \& Lingard, 2012). There is a need to examine competence from the perspective of the team rather than the competence of the individual members of the team, as competent individuals can form incompetent teams (Boreham, 2004; Hodges \& Lingard, 2012). This study was aiming to 
explore OR-PACU handovers whereby effective teamwork and collaboration can enhance the process. The concept of collective competence is well suited to unpack how individuals function in a team towards a shared goal and specifically the shared goal of an effective OR-PACU handover.

\section{Literature review}

\section{Search strategy}

The electronic databases PubMed, Medline, Ovid, CINAHL, Elsevier and Google Scholar were searched to explore the literature related to the OR-PACU handover process. The key words used were: "OR-PACU handovers", "postoperative handoffs", "interprofessional collaboration", "interprofessional communication", "handover tools", "perioperative”, "operating room", "postanaesthetic care unit", "perianaesthetic care", "recovery room”, "information transfer", "transfer of care", and "postoperative checklist". The keywords were used alone and in combination. The Boolean operators "or", "and" and the delimiter"/" were used to combine the keywords. Articles not specifically related to OR-PACU handovers but related to communication, teamwork, interprofessional collaboration and patient safety in the perioperative context were also considered. The inclusion criteria were set to include peer-reviewed journal articles published in the English language from 2007-present. The timeline reflects the introduction of the WHO Surgical Safety Checklist in 2007, a checklist used to support communication and transfer of information in perioperative practice. The timeline also reflects the Joint Commissions National Plan (2006) and the Required Organizational Practice by Accreditation Canada (2011) requirement for a standardized approach to patient handovers. These national directives increased interest in this safety approach. Hand searches of articles 
found in the bibliography of the selected articles were also implemented. The entire search yielded 187 articles that were reviewed for relevance. Full text was available for 172 articles out of which 76 were not relevant to postoperative handovers and 28 included editorials, short letters, or conference abstracts and were excluded. The 70 relevant articles were further refined for relevance to this study and 50 articles related to OR-PACU handovers were included in this review.

An environmental scan was also conducted via a distribution email list to the Perioperative Nursing Educators Canada (PNEC) group which includes clinical educators and managers within the perioperative setting across Canada to enquire about existing OR-PACU handover tools, guidelines and policies. Fourteen PNEC members from across the country responded to the email of which 12 responses included an attached handover policy or a tool specific to the institution. Of the 12 , there were five informal handover tools relevant to ORPACU handovers. The tools were mostly relevant to anaesthesia and PACU nurses, and were developed either by nursing or anaesthesia providers for the purpose of the hospital accreditation. Only one tool was applicable to the surgeon, anaesthesiologist and the OR nurse. There was no specific data or information available on the development, implementation, uptake and evaluation of the handover tool form the institutions. The information received from the PNEC group provided details of the current local practice within the Canadian context.

\section{Conceptual definition: Handover}

Handover can be defined as a "transfer of professional responsibility and accountability for some or all aspects of care for a patient or group of patients, on a temporary or permanent basis" (Canadian Medical Protective Association (CMPA), 2013). A postoperative handover can 
be defined as a transfer of professional responsibility and accountability for some or all aspects of care of the surgical patient in the immediate postoperative period. For the purpose of this study, handover will refer to postoperative handover, from the OR to PACU or an ICU, unless specified otherwise.

Handovers are dynamic, often occur in a rapid changing environment and the information communicated is at the discretion of the provider of the handover (Nagpal, Vats, Ahmed, Vincent, \& Moorthy, 2010a). Studies confirm that clinical handovers are often unstructured, incomplete and prone to communication failures (Botti et al., 2009; Manias, Geddes, Watson, Jones, \& Della, 2016; Manser, Foster, Flin, \& Patey, 2013; Manser et al., 2010; Milby et al., 2014; Nagpal et al., 2010a; Piekarski et al., 2015; Siddiqui et al., 2012).

\section{Interprofessional communication and teamwork}

Handovers require effective interprofessional communication and collaboration (Botti et al., 2009). There is evidence that poor communication is linked to poor patient outcomes and specifically the omission of items during transfer of care points (Sharit, McCane, Thevenin, \& Barach, 2008). Literature on the evaluation of communication practices during handovers is related to assessing the amount and the accuracy of the information transferred (Milby et al., 2014; Nagpal et al. 2010a; Siddiqui et al., 2012). Few studies assess communication practices in relation to team behaviour and its effect on the handover quality (Manser et al., 2010; Manser et al., 2013; Mazzocco et al., 2009; Nagpal et al., 2010b; Sharit et al., 2008).

In a qualitative study exploring transfer of information during a sign-out report in a paediatric intensive care unit (PICU) and PACU setting, Sharit et al. (2008) provided insight into interprofessional communication practices in the perioperative setting. Sharit et al. (2008) found 
work experience, lack of effective tools, and cultural background were contributing factors to communication failure. Furthermore tension between health care providers led to frustration and hindered effective collaboration and teamwork.

Effective teamwork and collaboration enhance patient experience and allow for a coordinated effort to recovery (Crosson, 2015). Mazzocco et al. (2009) reported that surgical teams who exhibited less or infrequent teamwork were at increased risk for complications or death. The retrospective chart review measured 30-day outcomes to examine the association between teamwork and patient outcomes. The results demonstrated that behaviours exhibiting less teamwork were linked to negative outcomes and were frequently observed during information sharing throughout the intraoperative and the postoperative phases suggesting, that surgical teams would benefit from a structured team training program (Mazzocco et al., 2009).

Communication behaviours and teamwork during handovers were also explored by Manser et al., (2013). In their cross sectional observational study, Manser et al. (2013) observed communication practices in 117 intraoperative handovers in three different settings. The communication practices were compared with the quality of the handover. There was a significant difference among the participants in the dominant communication practices such as information giving, receiving and acknowledging. Manser et al. (2013) identified four communication behaviours: information giving, followed by assessment, acknowledgement, planning and decision making. Assessment rather than information seeking during the handover was correlated with higher quality scores from the receiving participants, whereas there was no significant relationship found between communication behaviour and handover quality with the transferring participants. These results signify the variability in handover and the further need to 
explore the roles participants play during handover towards developing effective strategies to improve communication.

Communication behaviours were also discussed by Nagpal et al. (2010b) in their quantitative study which used failure mode and effect analysis (FMEA), a method frequently used to evaluate the health care process by a multidisciplinary team, to identify and evaluate potential failure modes in surgical care related to communication. Although most failure modes were seen in the preoperative phase, the most important failure in the postoperative phase was inadequacy of information, specifically related to postoperative care and management. The members of the multidisciplinary team involved in this study $(n=15)$ agreed that a systematic approach to potential risk assessment of the surgical process increased their awareness of patient safety and can provide valuable information towards the development of interventions.

After reviewing teamwork and communication practices between anaesthesia providers, Smith and Mishra (2010) found that intraoperative information was often underreported and unspoken. Implicit strategies and approaches were frequently employed as a part of the handover report. Smith and Mishra created a table of recommendations to improve handover practices based on findings from the observations of 45 handovers between the anaesthetists and the PACU nurses in their qualitative study. The recommendations included: postoperative handover starts only after the PACU nurse settled the patient and is able to give full attention to the anaesthesia provider during the report; anaesthesia provider remains in the PACU until the patient is stable; anaesthesia provider reviews the patient before discharge, and the PACU nurse is provided with clear postoperative instructions. Smith and Mishra also emphasized the need for attention to non-verbal and non-informational communication with the patient. 


\section{Factors influencing handover}

There are many factors that affect communication and teamwork during a handover. These include environmental, behavioural and cultural factors. Van Rensen et al., (2012) in their quantitative study explored self-reported multitasking during a handover. In addition to a nationwide questionnaire sent out to various HCPs, Van Rensen et al. also observed a total of 101 OR-PACU handovers in two settings. The aim of the study was to observe the overlap of activities related to handover such as information and equipment transfer and if these activities happened simultaneously or sequentially. The results revealed that the majority of the activities related to handovers happened simultaneously (43 percent of the self-reported participants and 65 observed handovers). The results also revealed that sequential handover was preferred by a majority of the participants.

A framework to examine the factors affecting clinical handovers was developed by Botti et al. (2009). The framework was tested in three PACU settings and was based on five concepts associated with the healthcare environment: clinical governance, engagement, ecological variety, safety culture and team climate, and sustainability. Botti et al. (2009) argued that many interventions directed to improving handovers fail because of the lack of attention to interprofessional communication practices and the multifaceted influences that affect handovers. The consideration of cultural and informal elements prior to the successful implementation of handover strategies that improve patient safety was also supported by Smith, Pope, Goodwin and Mort (2008). In their qualitative study aiming to describe handovers between the anaesthetists and the PACU nurses, Smith et al. (2008), observed 45 handovers and found that the handover process was dependent largely on mutual trust, power balance or differing expectations between the handover participants. Ambiguous roles and conflicting expectations between team members 
were also key elements affecting the handover process in a qualitative study by McElroy et al. (2015). Differences in priorities, institutional culture and relationships were also shown to potentially compromise patient safety during handovers (McElroy et al., 2015).

Another interesting observational study using descriptive and correlational design, explored the factors that affect the handover and memory during the handover (Randmaa et al., 2015). Randmaa et al. found, that handovers are often full of interruptions which occurred in $77 \%$ of the observed handovers and furthermore, unclear information was provided in $70 \%$ of handovers. Beyond studying interruptions and the general description of a handover process, Randmaa et al. also studied the factors that affect what the receivers remember after the handover. There was a significant relationship between the lack of structure, duration of the handover, interruptions during the handover and the information remembered. The overall results suggested that short and structured handovers were related to increased memory retention.

Arenas, Tabaac, Fastovets, and Patil (2014) recommended the use of a standardized mnemonics to facilitate the handover process. The case series study examined 50 handovers and investigated information recall after a handover. Arenas et al. found that undivided attention during a handover was significantly associated with items remembered by the receiver.

\section{Handover quality}

Overall 11 studies assessing the quality of the handover process were considered under this category. The handover quality in most studies is assessed by observations or an interview process and leads to the development of an assessment tool or a handover protocol. In one qualitative study, Nagpal et al. (2010a), used semistructured interviews with 18 HCPs to examine issues and problems surrounding handovers. The findings suggest that handovers are often 
incomplete and inconsistent and participants were all in agreement that handovers need to be structured. Informed by these findings, Nagpal et al. (2011), created a handover protocol that was validated by 50 HCPs (surgeons, anaesthesia providers, and PACU nurses) across three hospital sites in vascular and general surgery. The results showed improved handover structure with the implementation.

A checklist created specifically for the purpose of a prospective observational study was developed by Milby et al., (2014). The aim of the study was to analyze the transfer of information from anaesthesia providers to PACU nurses. The checklist included 59 items. The study included 790 handover observations collected over a two month period and the participants were blinded to the checklist content. The results revealed that items least reported were postoperative pain management (12\%), antibiotic therapy (14\%), fluid management (15\%), and the American Society of Anaesthesiologists (ASA) physiological status (7\%).

Siddiqui et al., (2012) also found that important information is often not communicated during the handover. The authors' prospective observational study examined 526 handovers over a period of 2 months. Similar to Milby et al., (2014), the participants were blinded to the 29 items of the checklist. The results indicated that only five items were consistently reported in over $80 \%$ of the handovers: type of surgery, analgesics given, difficult intubation, ST-wave changes, and comorbidities. Siddiqui et al. (2012) was also concerned with understanding what information was important to be reported by the participating anaesthesiologists and the PACU nurses. There was a difference in opinion in the items considered important between the participants resulting in inconsistent transfer of information during handovers. 
A different approach was undertaken in a study aiming to develop a rating tool to assess the quality of a handover process (Manser et al., 2010). The rating tool, piloted on 126 handover observations in three different settings, included 19 items and evaluated information transfer, teamwork and the overall quality of the handover. The analysis revealed that the rating tool provided great insight into the handover quality in the areas of information transfer, shared understanding and the working atmosphere. However, aside from OR-PACU handovers, which focused on the anaesthesiologist and the PACU nurse only, the data included handovers form PACU to the floor and from paramedics to the emergency department.

\section{Standardized handover process}

Overall 23 studies aiming to assess the impact of implementation, adaptation and evaluation of a handover protocol or a standardized process were considered under this category. Table 1 provides further details on the selected articles.

Table 1. Literature assessing the impact of an implementation, adaptation and evaluation of a postoperative handover protocol

\begin{tabular}{|c|l|l|l|l|}
\hline $\begin{array}{c}\text { Type of } \\
\text { Hanodver }\end{array}$ & $\begin{array}{c}\text { Prospective Pre- } \\
\text { Post Interventional }\end{array}$ & $\begin{array}{c}\text { Prospective/ } \\
\text { Interventional } \\
\text { Design }\end{array}$ & $\begin{array}{c}\text { Randomized } \\
\text { Controlled Trials }\end{array}$ & $\begin{array}{c}\text { Quality } \\
\text { Improvement } \\
\text { Methods }\end{array}$ \\
\hline & $\begin{array}{l}\text { Craig et al., 2012 } \\
\text { Caruso et al., 2015 } \\
\text { Joy et al., 2011 }\end{array}$ & $\begin{array}{l}\text { Agarwal et al., } \\
2012\end{array}$ & & \\
Chen et al., 2011 & & \\
KR-Paediatric & Fabila et al., 2016 & Kim et al., 2012 & & \\
ICU or PACU & Funk et al., 2016 & Mistry et al., & & \\
Hanodvers & 2011 & Piekarski et al., & & \\
& & 2015 & & \\
& & 2015 & & \\
\hline OR-ICU & Yang \& Zhang, & & Salzwedel et al., & \\
Hanodvers & 2016 & 2016 & \\
\hline
\end{tabular}




\begin{tabular}{|c|c|c|c|c|}
\hline & $\begin{array}{l}\text { Petrovic et al., } \\
2012\end{array}$ & & & \\
\hline $\begin{array}{l}\text { OR-PACU } \\
\text { Hanodvers }\end{array}$ & $\begin{array}{l}\text { Kitney et al., } 2016 \\
\text { Napgal et al., } 2013 \\
\text { Petrovic et al., } 2015 \\
\text { Potestio et al., } 2015\end{array}$ & $\begin{array}{l}\text { Weigner et al., } \\
2015\end{array}$ & $\begin{array}{l}\text { Salzwedel et al., } \\
2013 \\
\text { Robins \& Dai, } \\
2015\end{array}$ & $\begin{array}{l}\text { Boat \& Spaeth, } \\
2013\end{array}$ \\
\hline
\end{tabular}

Four studies focused on all team members, OR and PACU nursing, anaesthesia providers, and surgical providers (Caruso et al., 2015; Craig et al., 2012; Petrovic et al., 2015; Yang \& Zhang, 2016). Three studies focused on and evaluated the role of the anaesthesia provider during the handover and did not account for the other members of the team participating during the handover process (Joy et al., 2011; Salzwedel et al., 2016; Piekarsky et al., 2015). Still other studies included anaesthesia, surgical team, PACU nurses and other allied health care professionals (HCPs), such as internists and ICU attendants, with the exception of the OR nurse (Chen, Smith, \& Mistry, 2011; Fabila et al., 2016; Funk et al., 2016; Mistry et al., 2008; Vergales et al., 2016). Few of the studies included only anaesthesia and the surgical team members (Chen et al., 2011; Bonifacio et al., 2013; Zavalkof, Razack, Lavoie, \& Dancea, 2011). Others included only the anaesthesia provider and the PACU nurse (Boat \& Spaeth, 2013; Kitney et al., 2016; Potestio, Mottla, Kelley \& DeGroot, 2015; Robins \& Dai, 2015; Weigner et al., 2015). Coleman, Redley, Wood, Bucknall, and Botti (2015) assessed only PACU nurses perceptions with handovers and Kim et al's. (2012) study, relevant to handovers for paediatric patients following otolaryngology-head-neck surgery requiring postoperative airway management, included only the medical team led by a senior medical physician .

A tailored standardized handover protocol to improve handovers in the neurosurgical ICU was studied by Yang and Zhang (2016). Their pre-test post-test study involved a handover tool 
and a handover pathway developed by the core team members informed by the literature, evidence and expert input. There was 168 handovers included in the study and unlike other studies that focus on handover quality rather than the quality of patient care, Yang and Zhang also focused on the effect standardized process has on short term patient outcomes. Their results yielded improved short-term patient outcomes by significantly decreasing the duration of mechanical ventilation of patients in the first six hours postoperatively. The implementation of the handover process significantly improved teamwork and the attendance of the team members such as the surgeon during the report.

Improved communication and teamwork with the use of a standardized process was also found by Nagpal et al. (2013). The prospective pre and post-test study was aimed at assessing the implementation of an OR-PACU handover protocol in general and vascular surgery. The protocol involved the implementation of a handover protocol called Proforma and included a standardized process. The results revealed a decrease in the handover duration and an increase in handover satisfaction. The handover process included anaesthesia providers and members of the surgical team. OR nurses were not part of the handover process.

A standardized process was also found to reduce distractions during handover (Chen et al., 2011). Chen et al. explored the adaptation of a communication intervention over a period of time in a paediatric setting by observing 30 handovers. The results showed increased presence of the surgical team, reduction of nonessential distractions and improvement in patient centered information transfer with the implementation of the intervention.

Boat and Spaeth (2013) led a quality improvement study to improve their handover process after realizing that inconsistencies and lack of a standardized process during anaesthesia 
handoffs and anaesthesia to PACU nurses handoffs were the causes for three negative consequences: time inefficiencies, patient and family dissatisfaction and a medical error leading to patient harm. The postoperative handoff portion of the project, during which the plan-dostudy-act (PDSA) cycle was implemented, consisted of anaesthesia providers and PACU nurses. The project led to the development of a PACU handover checklist, which was laminated and provided to the anaesthesia team. The checklist consisted of 11 items and was used for training purposes. Boat and Spaeth (2013) found there was an overall satisfaction with the created checklist.

Improved satisfaction was also demonstrated by Coleman et al. (2015). who conducted a study aiming to evaluate the experiences of and the adaptation of a standardized OR-PACU handover tool. Coleman et al. used a descriptive, naturalistic design to explore PACU nurses perceptions of anaesthesia- PACU nurse handover practices. There were two focus groups and interviews with 17 PACU nurses. Results revealed factors such as interpersonal relationships, moods and behaviours between the PACU nurses and the anaesthesia providers as well as organizational factors and exposure to handover tools as important factors in the successful adaptation of a standardized handover process.

Significant improvement in the quality and the quantity of the information being transferred during OR-ICU handovers with the implementation of a standardized checklist was also found by Salzwedel et al. (2016), Their controlled randomized study included 121 handovers and compared the items handed over between the anaesthesiologist, the ICU physician and the ICU nurse. The results showed a positive increase in the patient and content specific information being transferred with the use of a checklist. Similar results were found in a randomized controlled study by Salzwedel et al. (2013), which observed 123 OR-PACU 
handovers, 40 pre-intervention and 80 post-intervention. However, both studies were specific to anaesthesia providers and did not include members of the surgical team or OR nursing.

In their randomized study, Robins and Dai (2015), observed 52 handovers, 26 pre and 26 post-intervention, and found that the use of the checklist can increase the accuracy and adequacy of the handover. The checklist implementation resulted in a reduction of follow-up phone calls to anaesthesia for information. In contrast, Petrovic et al. (2012) found that although insignificant, there was no decrease of post-handover follow up phone calls and pages following the implementation of a handover protocol in their prospective un-blinded study. Petrovic et al. (2012) created and evaluated a handover protocol for patients coming from the OR to a cardiac surgical ICU (CSICU). The handover protocol was developed with input from all members involved with these types of handovers, such as CSICU nurses, nurse practitioners, physician assistants, internists and anaesthesia and surgical providers. With the implementation of the protocol, the presence of team members during the postoperative handover increased from zero to $68 \%$. There was a significant decrease of parallel conversations during the report and there was a slight increase in the handover duration (one minute). Information sharing improved with the surgical team report, but was insignificant for the anaesthesia report. There was an overall increased satisfaction with the handover among the CSICU nurses.

In a later study, Petrovic et al. (2015) further described the implementation of their previously developed OR-CSICU handover protocol to a PACU environment. During this prepost-test interventional study, Petrovic et al. observed 103 handover, 53 pre-intervention and 50 post-intervention, with the aim to evaluate the OR-CSICU handover protocol in an adult PACU. Petrovic and colleagues implemented a variety of educational modalities prior to rolling out the protocol and the results showed a 77\% reduction of communication failures with the 
implementation of the standardized handover process. There was also an increase in participation of team members during the handover, especially the surgical providers.

Weigner et al. (2015) set out to improve postoperative handovers by combining simulation based interprofessional training of anaesthesia providers and PACU-RNs, a didactic webinar, post-training performance feedback towards the implementation of an electronic handover form which used the SBAR structure (Weigner et al., 2015). Weigner et al. observed 981 handovers, 389 in a pediatric PACU and 592 in an adult PACU. An overall improvement in the interprofessional handover was found with the implementation of a multimodal handover intervention in HCPs who received simulation training. The results also showed that the effect of the intervention was evident 3 years post-implementation in the adult PACU.

Many studies found that the duration of the handover remained unchanged with the implementation and use of a standardized handover process (Boat \& Spaeth, 2013; Caruso et al., 2015; Funk et al, 2016; Petrovic et al., 2015; Robins \& Dai, 2015; Salzwedel et al., 2016; Zavalkoff et al., 2011). Two studies found that the duration of the handover decreased with the use of a checklist (Caruso et al., 2015; Nagpal et al., 2013). In contrary, Potestio et al. (2015) led a quality improvement study focused on anaesthesia residents to PACU nurse handoff and found that the time was significantly increased in the group which used the checklist during the handover. The study focused on assessing 100 postoperative handovers and involved anaesthesia residents, 50 before and 50 after the implementation of a handover checklist. The results revealed that the residents addressed more information and spent more time on each item of the checklist if the handover took longer. 
In summary, research related to assessing the impact of an implementation, adaptation and evaluation of a handover protocol or a standardized process suggests that standardization of the OR-PACU handover not only improves the quality but also quantity of the information reported. For example the depth of patient information and the specific information reported for each patient (Nagpal et al., 2013; Salzwedel et al., 2016). Agarwal et al. (2012) reported a decrease in postoperative complications and improvement in postoperative outcomes in paediatric cardiac patients. The decrease was related to effective communication and a significant reduction of omitted information during the OR-Paediatric ICU handover which lead to an increased number of early extubation and a positive effect on postoperative patient outcomes. Standardization in handovers improved communication and teamwork and many studies reported a decrease in medical errors and increase in the number of items discussed (Joy et al., 2013; Nagpal et al., 2013; Petrovic et al., 2015; Potestio et al., 2015; Robins \& Dai, 2015; Salzwedel et al., 2016; Weinger et al., 2015). Studies also demonstrated an increased satisfaction of PACU nurses with the handover following the implementation of a standardized process (Nagpal et al., 2013; Petrovic et al., 2015; Potestio et al., 2015; Robins \& Dai, 2015). Robins and Dai (2015) also found a decrease of call-backs to clarify information following a standardized handover. Current research focuses on measuring the quality of the handover rather than measuring the outcomes of the handover and how a standardized process affects teamwork beyond the reporting of the various team members who participate in the process (Petrovic et al., 2015).

\section{Communication mnemonics}

The use of simple mnemonics to accurately convey information has been recommended and widely adapted as a part of a standardized handover process (Amato-Vealey, Barba \& 
Vealey, 2008; Bonifacio et al., 2013; Caruso et al., 2016; Grover \& Duggan, 2013; Iacono, 2009; Nasarwanji, Badir, \& Gurses, 2016; Sandlin, 2007; Wheeler, 2015). There are many mnemonics discussed in the literature. Nasarawanji et al. (2016) found 24 mnemonics specific to a handover and synthesized the information to represent what should be reported during the handover. The information that should be reported was clustered into 12 key themes such as introduction, patient information, assessment, situation, plan of care, recommendations, background and history and could be to customize a standardized handover communication process (Nasarawanji et al., 2016).

One of the most recognized approaches to improving communication is the situation, background, assessment, and recommendation (SBAR) technique (Amato-Vealey et al., 2008; Iacono, 2009; Grover \& Duggan, 2013; Nasarawanji et al., 2016; Radmaa et al., 2016). The SBAR is user friendly and conveys relevant and important information between various health care team members (Iacono, 2009). SBAR is versatile and can be applied to any clinical situation. The utilization of SBAR was found to minimize potential patient safety risks (AmatoVealey et al., 2008). Studies have also utilized the SBAR structure to analyze data related to communication process or to adjust the format to create individualized interventions (Bonifacio et al., 2013; Caruso et al., 2016; Fabila et al., 2016; Funk et al., 2016; Grover \& Duggan, 2013; Kitney et al., 2016; Weigner et al., 2015).

Other frequently discussed mnemonics to standardized handover communication are listed in Table 2 (Bonifacio et al., 2013; Caruso et al., 2016; Kitney et al., 2016; Funk et al., 2016; Sandlin, 2007; Wheeler, 2015). Many, such as the SBARQ technique which expanded the traditional SBAR format, added the question option and provided an opportunity to ask questions and clarify the information communicated (Bonifacio et al., 2013; Funk et al., 2016). 
Table 2. Frequently used mnemonics during handovers

\begin{tabular}{|l|l|l|l|l|}
\hline \multicolumn{1}{|c|}{ SBARQ } & \multicolumn{1}{|c|}{ I-SBAR } & \multicolumn{1}{|c|}{ SHARQ } & \multicolumn{1}{c|}{ I PASS the } & \multicolumn{1}{c|}{ B Ps } \\
\hline Situation & Introduction/ & Situation & Introduction & Patient \\
Background & Identification & History & Patient & Precautions \\
Assessment & Situation & Assessment & Assessment & Plan of care \\
Recommendation & Background & Recommendations & Situation & Problem \\
Questions & Assessment & Questions & Safety concerns & Purpose \\
& Recommendation & & the & \\
& & & Background & \\
& & & Action & \\
& & & Timing & \\
& & & Ownership & \\
& & & Next & \\
& & &
\end{tabular}

Despite wide acceptance mnemonics have been problematized in the literature mainly because of the uncertainty of their initial development. The words used in different mnemonics may overlap and have different meaning, and there exists a lack of evidence that mnemonics actually standardize and improve a handover (Nasarwanji et al., 2016). Most mnemonics also lack comprehensiveness and do not necessarily prompt reporting of all the essential information during the handover (Nasarawanji et al., 2016). 


\section{Compliance with standardized handover process:}

Despite many positive results with the implementation of a standardized handover process, compliance with the implementation of a handover tool often remains a challenge. In their pre-post-test study Kitney et al. (2016) measured the compliance with I-SBAR during ORPACU handovers in two hospitals before and after an educational session. The results were mixed. One hospital showed increased compliance with the I-SBAR demonstrating improvement in some items being reported (cardiovascular, respiratory, analgesia and actions). The second hospital showed a decrease in reporting similar items such as respiratory, referral and responsibilities information with the use of the I-SBAR, but, an improvement in reporting for example communication difficulties. The results found that an educational session to introduce structured handover strategies may or may not improve the compliance with the intervention. Potestio et al. (2015) further found that only 4 out of the 50 post-intervention handovers observed reported on all of the items on the checklist. Similarly, in their controlled randomized study observing OR-PACU handovers from the anaesthesia providers to PACU nurses before and after the implementation of a checklist, Salzwedel et al. (2013) found that even with the use of the checklist only slightly over half of the items on the checklist were reported.

In contrast, despite mandatory implementation and close to $100 \%$ compliance with the use of a checklist in the perioperative setting, Urbach et al. (2014) found there was no significant reduction of mortality or complications with the checklist implementation. The study analyzed data from 101 hospitals three months before and after the implementation of the surgical safety checklist. 
Furthermore, many studies that describe the use or implementation of handover protocols or checklists, do not comply with the Joint Commission Center for Transforming Healthcare (JCCTH) guidelines for the development of handover tools and interventions (Pucher et al., 2015). The Joint Commission guidelines, known under the acronym SHARE (standardize, hardwire, allow, reinforce, and educate) have been developed by analyzing specific root causes within the handover process and address critical areas for successful implementation of the intervention, such as implementation, reinforcement and education (JCCTH, 2014; Pucher at al., 2015). Pucher at al. (2015) found only one study complied with all five components of the SHARE guidelines (JCCTH, 2014). Many studies failed essentially because of the lack of follow-up and exploration of the impact of their intervention on patient outcomes (Pucher at al., 2015).

\section{Summary}

Within this chapter an overview of literature related to OR-PACU handovers, specifically interprofessional communication and teamwork, factors influencing the handover, the handover quality, a standardized handover process, communication mnemonics, and the compliance with standardized handover process was provided.

A standardized process during information transfer at points of care transition is a required organizational practice by the Accreditation Canada 2016. Overall, there is a growing body of literature examining OR-PACU handovers; however, there continues to be a gap in the literature where all team members are considered and involved with the handover process. Further understanding of the underlying factors that support a team approach to handover is critical in developing effective standardized process. 


\section{CHAPTER 3: METHODOLOGY}

This study used a descriptive qualitative approach which included the observations of handovers of patients coming from the OR to PACU, and by conducting informal interviews and a focus group session with health care professionals involved with OR-PACU handovers on a regular basis. This chapter will provide details of the methods used to conduct this study including participants' details, recruitment strategies, data collection and management, data analysis, ethical considerations and the establishment of rigor.

\section{Research question}

The objective in this qualitative descriptive study was to gain insight into the processes, roles and factors that underpin effective OR to PACU handover. To meet the objectives, surgical patient safety related to OR-PACU handovers were investigated in the PACU to gain insight into the following domains with the following research questions:

1. Patient Safety Practices and Work Dynamics

a. What are the factors and processes that affect the safety practices in the PACU during handovers?

b. What is the relationship between surgical patient safety practice(s) and handover behavior amongst PACU/OR health professionals?

2. Team Handover

a. What information do various professional members perceive as important during the handover discussion?

b. How does information perceived as important influence what is being reported during handovers? 


\section{Research design and method}

Qualitative research encompasses many approaches and is different from quantitative research as it depends predominantly on linguistic data and using a meaning-based form of analysis (Elliot \& Timulak, 2005). There are many qualitative approaches that vary technically and theoretically, such as phenomenology, ethnography or grounded theory (Sandelowski, 2000; Smith, Bekker \& Cheater, 2011). Qualitative description is a qualitative approach that has increased in popularity in the last few years especially in studies requiring a descriptive account of phenomenon (Sandelowski, 2000). This is especially valuable as more traditional qualitative approaches are increasing in complexity and may not support the need of the researcher (Sandelowski, 2000).

Qualitative description provides rich description and in-depth understanding of the phenomenon as it presents in its natural environment (Sandelowski, 2000; Neergaard, Olesen, Andersen, \& Sondergaard, 2009). As opposed to other qualitative approaches, qualitative description allows for less interpretation and as such permits the researcher to stay near or true to the data (Sandelowski, 2000; Sandelowski, 2010). In general, with qualitative description, data collection takes place in the natural setting without pre-selection and manipulation of the phenomenon and the focus is on data richness and quality rather than on the number of participants (Sandelowski, 2000; Smith et al., 2011). The credibility of the research further depends on how consistent the study purpose is with the appropriate sample selection, data collection and analysis methods (Sandelowski, 2000). The final result of qualitative descriptive research is a description of the phenomena in an organized manner that best depicts the content (Sandelowski, 2000). 
Qualitative description is situated in the naturalistic paradigm - the philosophical foundation of this method (Sandelowski, 2000). It is a continuous, flexible and adaptable design best suited for this study as it provided an approach to support in-depth understanding of the factors and processes that affect the OR-PACU handover.

\section{Research paradigm}

This study was conducted within the naturalistic paradigm which is often referred to as constructivist paradigm (Loiselle, Profetto-McGrath, Polit, \& Beck, 2011). The researcher in this paradigm believes that reality is not fixed but rather exists within the context and that multiple truths and realities are possible (Loisell et al., 2011; Sandelowski, 2000). Knowledge and insight in naturalistic paradigm are generated through voices and interpretations of those involved with the phenomenon (Loisell et al., 2011). The naturalistic inquiry takes place in the natural setting and is concerned with exploring human complexity and understanding the lived experience (Loisell et al., 2011). Furthermore, the relationship and interactions between the researcher and the participants play an important role on data gathering in naturalistic inquiry (Loisell et al., 2011). The naturalistic paradigm was well suited for this study which was aiming to gain a rich and in-depth understanding of the OR-PACU handover from the perspective of the participants who were involved with the process.

Setting. The study took place in a large urban teaching hospital in Toronto, Ontario, in the PACU department. The facility completes on average 8000 cases per year or an average of 23-28 cases per work-day in the main OR department in the specialties of orthopaedics, general surgery, gynecology, uro-gynecology, urology, ophthalmology, maxillo-facial surgery, ear-nose and throat (ENT) and plastic surgery. The surgical mix consists of $60 \%$ patients that stay in the 
hospital after the surgery (inpatients) and $40 \%$ patients that are discharged on the same day of their surgery (day surgery patients).

The majority of surgical patients in the facility are being admitted three to four hours prior to the scheduled procedure. Few surgical patients come to the OR directly from the floor, emergency department (ER), or the ICU. The same day admit process requires the patient to obtain an admitting sheet and a hospital card in the admitting department located on the main floor. The patient then continues to the surgical admitting room (SRA). The SRA is within close proximity to both the PACU and OR. Patients in the SRA are admitted by administrative clerks and volunteer personnel before being checked in by the PACU nurse. The patient is further assessed by the anaesthesia provider, the OR nurse, and a member of the surgical team before coming to the OR.

Following the completion of surgery, the patient, accompanied by the members of the perioperative team, is transferred to the PACU department. The PACU department, where the study took place, is adjacent to the OR department and has a capacity of 17 postoperative patient bays and eight postoperative chairs. Depending on the health status of the patient and the type of surgery postoperatively patients leave the OR either on a stretcher, a bed or in a wheelchair. Patients on a stretcher or a bed are recovered in the PACU bays, whereas patients leaving the OR in a wheelchair are typically recovered in the chairs. The postoperative patient is transferred from the PACU to the designated hospital destination or discharged home upon meeting the discharge criteria.

Participants. This study used a convenience and a maximum variation sampling to recruit participants. Convenience sampling is a method in which the researcher recruits 
participants who are most convenient and readily available (Loisell et al., 2011). Maximum variation sampling allows for exploring the diversity of the observed phenomenon (Loiselle et al., 2011). As such, the aim was to recruit patients from a variety of surgical services regardless of their acuity and admission status. Convenience maximum variation sampling was also used to attract perioperative healthcare professionals and surgical specialties.

The number of health care participants in this study was closely related to the total number of staff within the OR, PACU and anaesthesia departments in the institution. Having all HCPs participate was ideal given the random daily assignments for each OR and unpredictability of who would be present during the postoperative handover. In contrast, this was not applicable for the surgical members who were rarely present during the handover and therefore were consented on an as necessary basis.

The researcher in qualitative studies aims to achieve a holistic and comprehensive understanding of the phenomenon of interest. The aim with qualitative descriptive research is to gain data saturation by rich description of the phenomenon (Sandelowski, 2000). Data saturation allows for better transferability of the findings and relates to the sample adequacy at which point no new information is acquired (Loiselle et al., 2011; O’Reilly \& Parker, 2013; Sandelowski, 2000). The aim was to spend 30-50 hours of observation in the PACU environment. The preliminary analysis revealed that 50 to 60 handovers provided sufficient understanding of the OR-PACU handover process. Furthermore, this number was supported by other studies exploring handovers while averaging between 50 and 500 handovers per study (Caruso et al., 2015; Gurses et al., 2012; Manser et al., 2010; Milby et al., 2014; Nagpal et al., 2013; Randma et al., 2015; Siddiqui et al., 2012; Zavalkof, Razack, Lavoie, \& Dancea, 2011). 
Inclusion and exclusion criteria. Participants had to be between 19-85 years of age and English speaking. The age limit was consistent with the age range of patient and staff participants readily available in the institution. The English language was chosen as it would be difficult to provide an official English translator to non-English speaking participants due to availability of translation services. The eligible participants included all surgical patients (day admit patients and in-patients) scheduled for an elective surgical procedure in the main OR department from Monday to Friday between 07:30-17:00 hours. Emergency cases and surgeries finishing after 5PM were excluded from the study for feasibility reasons. ICU cases were excluded as the focus of this study was on OR-PACU handovers whereas ICU patients in the institution frequently bypass the PACU post-operatively to go directly to ICU.

The healthcare professional participants were any members of the perioperative and postanaesthestic care team including registered nurses (RNs), anaesthesia assistants (AAs), anaesthesiologists and surgeons including clinical fellows and residents involved in an OR/PACU handover.

\section{Recruitment strategy:}

Health care professionals. Recruitment posters were placed in the common areas in the perioperative department: surgical, nursing, and anaesthesia lounges, locker rooms, the PACU and on the doors of the sterile core inside the OR department (See Appendix 2: Recruitment Notice HCP). A brief introductory email with the recruitment notice was sent to anaesthesia, OR and PACU nursing, and surgical staff by the departmental heads. An information session about the study was provided during dedicated in-service time for the OR nurses. The researcher was available in the perioperative department for two weeks between the hours of 7:30-16:00 on 
Monday to Friday to further provide detailed information about the study and answer any questions. Consent forms were provided to interested individuals and available at the OR desk, anaesthesia lounge and the PACU desk (See Appendix 4: Consent Form HCP). For convenience, consent drop-off boxes were created and available during the recruitment period. Many HCPs were observed on more than one occasion throughout the data collection period and additional verbal consent was further obtained for all subsequent observations and informal interviews with individuals who already had given written consent (See Appendix 7: Additional Verbal Consent HCP). To be included in the study, all team members involved in the handover, including the patient, had to be willing to participate in the study. If a single member of the team chose not to participate in the study the entire handover was not eligible for observation. Similarly, if one member of the handover subsequently chose not to participate after initially agreeing, the data collected were removed from the analysis.

Focus group participants. HCPs who signed an informed consent were provided a number code and were also asked to participate in a focus group session in the second phase of the study. Interested participants were marked with extra number codes and added in a draw prior to the focus group session recruitment. A draw was made by the researcher from the interested HCPs aiming to select an equal number (2-3) of participants from each team (OR and PACU nursing, anaesthesia and surgery) to participate in the focus group session approximately one month prior to the pre-scheduled date. The selected participants were provided with two possible dates and consulted on their availability. Participants were further provided with information about the purpose and objectives of the focus group session as outlined in the focus group consent form (See Appendix 5: Additional Consent HCP: Focus Group). Available participants were informed that the session will be audiotaped and were asked to sign an 
informed consent specific to the focus group session. Focus group participants received a $\$ 20$ gift card and an appreciation letter as a token of gratitude for their time (See Appendix 11: Appreciation Letter Focus Group Particpants).

Patients. Recruitment posters for patients were placed in surgical pre-admitting area (PAU) and in the surgical admitting and interview rooms (See Appendix 1: Recruitment NoticePatients). The patients were recruited on the day of their surgery. Administrative personnel or the health care providers in the surgical admission room introduced the study briefly to surgical patients upon arriving and asked if they were interested in more information. Any surgical patient who expressed an interest to volunteer for the study was provided detailed information about the study by the researcher and was asked to sign an informed consent. Prior to signing the consent, the researcher explained all elements of the consent form, asked if they had further questions, and provided any additional clarification at the patient's request (See Appendix 3: Consent Form for Patients; \& Appendix 6: Additional Verbal Consent Patients). It was made clear that the researcher was not participating in any part of the handover itself and that the decision to participate in the study was voluntary and would not in any way delay or affect their quality of care. Patients were given on average 40 minutes to one hour to decide if they would like to participate before signing the consent and recruited patients were provided with a copy of the consent and assigned de-identified codes.

\section{Ethics}

Following ethical principles in qualitative research is especially important because the lives of participants are being studied and evaluated (Yeo, Moorehouse, Khan, \& Rodney, 2010). Issues of confidentiality and privacy, informed consent, participant-researcher relationship, 
anonymity and other possible ethical issues must be considered (Streubert \& Carpenter, 2011; Yeo, Moorhouse, \& Krahn, 2010).

Research can affect the individual or the society on a physical or physiological level and as such this study was guided by the three core principles of The Tri-Council Policy Statement 2 (TCPS2): Ethical Conduct for Research Involving Human Subjects (2014). These core principles are: respect for person, concern for welfare and justice (Canadian Institute of Health Research, Natural Sciences and Engineering Research Council of Canada, \& Social Sciences and Humanities Research Council of Canada, 2014). This study received approval from the Research Ethics Board (REB) of the institution (REB\# 15-0062E) and from the REB of the University (REB\# \# 2015-136).

Respect for person. The researcher has a moral and a legal obligation to consider and to respect autonomy of the participants. Autonomy is the individual right to make a free and independent decision and informed choice (Moorhouse, Yeo, \& Rodney, 2010). Guided by the principles of autonomy, the researcher was seeking free, informed and ongoing consent from all participants. The researcher also ensured that her relationship with the study participants remained free of coercion and undue influence.

Concern for welfare. The researcher is obligated to protect the participants from harm and any negative effect on mental, spiritual and physical health and on economic, social and physical circumstances (TCPS2, 2014). Guided by the principle of welfare the participants were informed that there are no foreseen risks involved with participating in the study and that there are no direct benefits to them. The researcher was also transparent by clearly stating the objectives and the purpose of the study. Concern for welfare also includes the concepts of 
privacy and confidentiality (TCPS2, 2014). Privacy is related to the individual right not to disclose health and personal information and confidentiality is related to protecting the disclosed information (Yeo et al., 2010). The participants were informed that the researcher will not interfere or intervene with their planned care and were informed regarding how privacy and confidentiality will be maintained.

Justice. The principle of justice is concerned with fair and equitable treatment while protecting vulnerability of participants (TCPS2, 2014). As such, all participants were treated with equal respect and consideration. Consideration towards possible imbalance of power between the researcher and the participants included the researcher use of layman language to explain the purpose of the study and when answering the participants' questions.

One possible ethical consideration was that the researcher was a staff RN (not in a position of authority) in the operating room in the institution where the study took place and therefore a co-worker of the potential participants. To avoid undue influence, self-referral, based on recruitment notices and emails rather than a personal approach was used to recruit participants. Every effort was made to further ensure that personal relationships with the participants did not influence their willingness to participate in the study. The researcher was not engaged in any activities related to patient care during the time as a researcher on the unit and clearly notified the staff about her role, i.e. researcher vs staff nurse during the data collection period. There was no relationship between the person obtaining the consent and the surgical patients. Every precaution was taken to ensure that the research activities did not in any way affect the regular activities of the perioperative department. For example, the researcher came to meet and recruit the patients at 6:30am in the morning not to interfere with the perioperative team who had to check the patients in before 7:30am. 
Personal and health information of participating patients only remained identifiable during the recruitment process. All personal and health information was stored separately from the consent forms according to a unique study ID code assigned to each participant to ensure anonymity. Only persons directly involved with the research study (thesis committee members) were able to access the collected data. Field notes, transcripts, and the list of the research code numbers/pseudonyms with the corresponding participant identifying information were stored in a locked cabinet in a locked office at the institution, separate from the consent forms. Any data obtained in this study was stored on the hospital network's secure server on password protected computers and database. De-identified data were stored on a hospital encrypted USB stick and the information was password protected. Audio-recordings from the focus group session were destroyed immediately following transcription to maintain confidentiality. All data and consent forms will be securely stored for seven years and then destroyed. No identifying information will be used in any publication or presentations.

\section{Data collection}

Data collection in qualitative descriptive research is aimed towards achieving a complete account of what happened, who was involved and where it happened (Sandelowski, 2000). Methods commonly employed to gather data in qualitative descriptive studies are observations, semi-structured interviews, focus group sessions and examination of documents and artifacts (Sandelowski, 2000). The data collection methods in this study were directed towards understanding the factors and processes affecting the OR-PACU handover. The methods were a combination of observations in the PACU environment, informal interviews with the staff and a focus group session. Analysis was also conducted on existing documents used to record or inform handovers, such as blank anaesthesia sheets and nursing handover transfer forms as well 
as policies and procedures related to surgical patient safety linked to OR-PACU handovers. The data were collected over a three month period by a single researcher.

A. Observations. The main focus of the observations was to observe the OR-PACU handover report of surgical patients in the immediate post-operative period. In particular, the focus was on the information communicated, the team communicating the report and the factors and processes affecting the report. Detailed notes from the observations included: the location, duration, content and order of the handover; contradictions, questions, and pattern of participation among team members. The field notes also included information on procedure type; participants' activities and relevant communication immediately before and after the handover; the structure and layout of the setting; patient passage through the PACU; detection and response to potential safety issues; interactions between team members and patients.

The data during the observations was collected on a Handover Data Collection Form. The form was developed by the researcher to facilitate the collecting of the large amount of data during the short time period allotted for the OR-PACU handover report. The form was informed by relevant literature examining OR-PACU handover, the existing anaesthetic sheet and the OR nursing transfer sheet used in the institution during the ORPACU handover report (Siddiqui et al., 2012).

The observations did not interfere with routine patient care and did not prolong the handover. The researcher required access to the patient medical chart, personal and health history during the observations to check accuracy of the information being relayed during the handover. The health and personal information was not used for identifying purposes or linked to the patient's name. 
B. Informal Interviews. Informal interviews were conducted by the researcher after the handover report observations had concluded and once the patient was settled. The researcher also engaged in informal conversations on a regular basis with the HCPs participating in the study over a period of two months. The main focus of the interviews was to gain understanding of the OR-PACU handover current practices and to clarify or ask questions of the report that was observed (See Appendix 9: Sample Questions: HCP). This allowed the researcher to follow up immediately following the observations in the field. Written notes taken by the researcher during the interview were transcribed immediately after the interview to assure accuracy.

Since surgical patients are often under the influence of the anaesthetic and are not actively involved in the OR-PACU handover process we investigated their perception on what information should be included in the report prior to surgery. In addition to HCPs, patients who agreed to participate were randomly engaged in a conversation and an informal interview by the researcher while in hospital before the surgery about their perceptions on information communicated during transfer of care points (See Appendix 8: Sample Questions: Patients).

C. Focus Group Session: In addition to observations and informal interviews, a sample of 8-10 participants from OR and PACU nursing, anaesthesia, anaesthesia assistants, and surgical teams were recruited to participate in a focus group session. The focus group session was held after the completion of the data collection and preliminary data analysis from the observations and informal interviews. The main objective of the session was to allow the participants to provide feedback on the preliminary findings of the study. 


\section{Data analysis}

Qualitative content analysis was used to analyze the data and followed the process outlined by Elo and Kyngas (2008). Content analysis is a flexible method that provides an objective, systematic approach to analysis of large volumes of data. Qualitative content analysis is the method of choice in qualitative description as it is also characterized by its reflexivity and interactive approach (Sandelowski, 2000). The aim of content analysis is to provide a broad understanding and description of phenomenon (Elo \& Kyngas, 2008). Content analysis is useful in describing, discovering and examining the focus, trends and patterns as related to an individual, a group, institution or a society (Hsieh \& Shannon, 2005; Krippendorff, 2004). Furthermore, content analysis is focused on context, meaning and consequences and is a useful method for providing understanding of communication and critical process (Elo \& Kyngas, 2008; Hsieh \& Shannon, 2005; Wilson, 2011). Content analysis can be used for data that comes from a variety of sources, such as observations, focus groups, narrative responses, videos, recordings, documents, or manuals (Hsieh \& Shannon, 2005; Krippendorf, 1980; Wilson, 2011).

Content analysis originated in the $18^{\text {th }}$ century and was initially derived from the communication theory (Vaismoradi, Turunen, \& Bondas, 2013). Despite being used as both, a qualitative and quantitative analytical method in the beginning of the $20^{\text {th }}$ century, it was considered primarily a quantitative tool (Hsieh \& Shannon, 2005). However, the use of content analysis in qualitative analysis was recognized and this method has been widely applied, especially in health research (Hsieh \& Shannon, 2005). Content analysis is widely used in communication, sociology, psychology, and business and has been gaining popularity in health care and education in recent years (Elo \& Kyngas, 2008; Graneheim \& Lundman, 2004; Hsieh \& Shannon, 2005). 
There are three approaches to content analysis: 1) inductive, also known as conventional, 2) deductive, also known as directed and 3) summative approach (Elo \& Kyngas, 2008; Hsieh \& Shannon, 2005). With the inductive approach, the categories are derived directly from the data and it is often used when there is a limited understanding or knowledge about the phenomenon (Elo \& Kyngas, 2008; Hsieh \& Shannon, 2005). The deductive approach uses existing theory or previous knowledge about the phenomenon to test or guide the analysis (Elo \& Kyngas, 2008; Hsieh \& Shannon, 2005). The summative approach involves counting and comparing words and codes to interpret the context of the data (Hsieh \& Shannon, 2005). The inductive approach to content analysis was best suited for this study for its ability to define the patterns, trends and relationships that emerged directly from the data collected during the observations, informal interviews and later the focus group session. It provided a broad understanding of the communication process and the critical issues surrounding the OR-PACU handover.

There are various processes to content analysis described in the literature and their relevance is mainly in the field of communication, journalism, library and political science, or quantitative research (Krippendorf, 1980; Wilson, 2011). The analytical process to content analysis as outlined by Elo and Kyngas (2008) was most appropriate for this study for its relevance to health sciences and nursing. The process is based on theoretical knowledge and relevant methodological literature and leads to categories that describe the phenomenon (Elo \& Kyngas; 2008). This process relies on high quality data and consists of three phases in which the data is reduced to smaller content categories (Elo \& Kyngas, 2008). The three phases of inductive content analysis as outlined by Elo and Kyngas (2008) and used in this study are: 1) preparation phase, 2) organizing phase and 3) reporting phase. 
Phase 1: Preparation. The preparation phase begins with selecting a unit of analysis and deciding if a manifest (the obvious) or a latent (the hidden) content will be analyzed (Elo \& Kyngas, 2008; Graneheim \& Lundman, 2004). Unit of analysis could be a variety of objects such as a letter, number, word, sentence, but also an entire interview and observation sets (Elo \& Kyngas, 2008; Graneheim \& Lundman, 2004; Vaismoradi et al., 2013). The unit of analysis selected in this study was the data which were collected during the handover observations, informal interviews and focus group session.

Manifest content refers to obvious and visibly available data where latent content relates to the infered meaning or the intrepretation of what the data present (Graneheim \& Lundman, 2004). This could be, silence, laughter, relationships and other hidden context (Elo \& Kyngas, 2008; Graneheim \& Lundman, 2004). Latent content requires a deeper level of abstraction and for the purpose of this study, which was aiming to achieve a descriptive account of the ORPACU handover, manifest content was decided to be more appropriate (Elo \& Kyngas, 2008; Graneheim \& Lundman, 2004).

The next step during the preparation phase consists of the researcher becoming immersed in the data. This includes transcribing and reading the data repeatedly to gain a sense of the whole (Elo \& Kyngas, 2008; Hsieh \& Shannon, 2005). As such, the researcher in this study personally transcribed all data collected during the observations, interviews and the focus group session. Following the transcriptions, the data was read and reread thoroughly. This allowed the researcher to become completely immersed in the data set while trying to make sense of what is happening, where, when, why and by whom (Elo \& Kyngas, 2008). 
Phase 2: Organizing. The organizing phase begins with open coding, creating categories and further grouping codes into higher categories that provide general description of the research topic (Elo \& Kyngas, 2008). This phase requires going back to the data to create notes and headings in a form of track changes in the margins of each transcribed data set. The notes (meaning units) and headings (condensed meaning units) were descriptive in nature and summarized the content. These were re-read several times, collected in a separate document and clustered into emerging codes. Emerging codes were further compared and grouped under similar or related categories that were freely developed initially. The process of analysis in this study is illustrated in Table 3. During this part of the analysis process, the researcher moves from specific to general understanding of the data (Elo \& Kyngas, 2008). The initial codes and categories were created to capture the main thoughts and impressions interpreted from the data. These categories were abstracted and were named to characterize the content. It was important that each category included data and codes that that were not only similar and related but also belonged to the category (Elo \& Kyngas, 2008).

Table 3. Examples of Data Analysis Process: Unit of Analysis, Meaning Unit, Condensed Meaning Unit, Code, Subcategory and Category

\begin{tabular}{|c|c|c|c|c|c|}
\hline $\begin{array}{c}\text { Unit of } \\
\text { Analysis }\end{array}$ & $\begin{array}{c}\text { Meaning } \\
\text { Unit }\end{array}$ & $\begin{array}{c}\text { Condensed } \\
\text { Meaning Unit }\end{array}$ & Code & Subcategory & Category \\
\hline $\begin{array}{l}\text { Handover } \\
\text { transcript } \\
\text { data }\end{array}$ & $\begin{array}{l}\text { The team had } \\
\text { to move tables } \\
\text { and equipment } \\
\text { out of the way }\end{array}$ & $\begin{array}{l}\text { Table and } \\
\text { equipment in the } \\
\text { way }\end{array}$ & $\begin{array}{l}\text { Physical } \\
\text { space }\end{array}$ & Environment & $\begin{array}{l}\text { Distractions } \\
\text { and } \\
\text { interruptions }\end{array}$ \\
\hline
\end{tabular}




\begin{tabular}{|l|l|l|l|l|l|}
\hline & $\begin{array}{l}\text { before the } \\
\text { report }\end{array}$ & & & & \\
\hline
\end{tabular}

Phase 3: Reporting data. Reporting data is the final stage of the inductive content analysis process as described by Elo and Kyngas (2008). This phase, in which creativity in a form of a model, map or a story line is encouraged, is concerned with reporting the findings from the previous phases (Elo \& Kyngas (2008). The hierarchical coding model has been chosen to organize the findings in this study as it best demonstrates the relevance of the findings to its audience. The findings from this study are presented in a hierarchical structure format in Chapter 4 Results. Definitions of each category and subcategory developed during the analysis process and exemplars of codes derived from the data were also included in an extraction table for illustration purposes in the results section.

\section{Rigor}

Rigor in qualitative research is concerned with the process in which results were achieved, in particular the appropriateness of the methods and the thoroughness of the research (Lincoln \& Guba, 1985). In qualitative descriptive design rigor can be demonstrated by means of credibility, dependability, confirmability and transferability (Elo \& Kyngas, 2008; Graneheim \& Lundman, 2004; Hseih \& Shannon, 2005; Sandelowski, 1993).

1. Credibility. Credibility refers to the confidence in the true value of the findings and how well the study addresses the intended focus (Elo \& Kyngas, 2008; Graneheim \& Lundman, 2004; Loiselle et al., 2011). Credibility can be established by various techniques, such as 
prolonged engagement and persistent observations, triangulation, external checks and researcher credibility (Loiselle et al., 2011; Streubert \& Carpenter, 2011).

Prolonged engagement. Prolonged engagement is considered to be one of the best approaches to enhance credibility and involves engaging in data collection activities over a period of time (Loiselle et al., 2011; Sandelowski, 1986; Streubert \& Carpenter, 2011). Persistent observation is closely related to prolonged engagement and enhances credibility by providing a depth of understanding of the situation relevant to the phenomenon (Loiselle et al., 2011). The data in this study was collected over a period of three months. During this time the researcher developed rapport and close relationships with PACU nurse and the various team members. This time allowed the researcher, who is familiar with the environment as a perioperative nurse, to expand the initial understanding of the culture and everyday workflow in the PACU setting. Prolonged engagement and persistent observation in this study allowed the researcher to collect credible data and further enriched the interpretation.

Triangulation. Triangulation is a method in which the researcher uses multiple sources and methods to shed light on the various perspectives (Creswell, 2013). The data in this study were collected by means of observations, informal interviews and a focus group session. Using different sources the researcher compares the data for consistency adding to the rigour of the findings (Creswell, 2013; Sandelowski, 1986). Further, health care members from various professions and patients from various surgical specialties were included in the study providing various views and perspectives. As such, the triangulation method and the data source triangulation provided a more complete and contextualized view of the phenomenon. 
External checks. The two key components in external checks are peer debriefing and member checks (Loiselle et al., 2011; Sandelowski, 1986; Streubert \& Carpenter, 2011). The principle behind external checks is to confirm the objectivity of the findings and whether the participants feel the findings are accurate (Streubert \& Carpenter, 2011). The method used in this study was a peer debriefing which took place during the focus group session. The focus group session participants were presented with the preliminary findings of the study and were asked for feedback and their experience with OR-PACU handovers (See Chapter 4 Results). The researcher also often consulted with the members of her thesis committee, who were experts in the field of qualitative research and the studied phenomenon, on the methodological aspect of this study. The committee members provided input throughout the study and were frequently consulted during the data analysis process.

Reflexivity. As a perioperative registered nurse I had an existing professional relationship with many of the participants and a personal interest in the studied phenomenon. This could theoretically affect the researcher's objectivity. As such, I was continuously examining my own professional experiences and practice and how these shaped my perceptions throughout the study by engaging in reflective journaling and writing notes following observations and interviews. I also ensured that my role was clearly defined to my colleagues. It was important to maintain selfawareness throughout this study by means of reflection and journaling. Self-awareness and the acknowledgement of the influences the relationship between the researcher and the participants have on the results are the main components of reflexivity which is an essential part of the qualitative research process (Kitto, Chesters, \& Grbich, 2008). Writing personal notes following the observations and interactions with participants also added to the credibility of the data. These methods allowed me to explore subjective perceptions, thoughts and feelings about the 
phenomenon (Ortlipp, 2008). Maintaining focus, being true and staying near to the data and frequent consultations with the thesis supervisor were other helpful strategies to examine the researcher's values and sociocultural position within the study and ultimately ensure credibility of the findings was achieved (Kitto et al., 2008).

2. Dependability. Dependability also known as auditability is closely related to credibility and confirmability of findings (Loiselle et al., 2011; Sandelowski, 1986). Dependability is concerned with data stability and the trustworthiness of the findings (Sandelowski, 1986). Dependability accounts for the potential instability and changes that may occur throughout the data collection period (Loiselle et al., 2011). Dependability in this study was considered by maintaining consistency during the informal interviews with various health care professionals and by openly addressing the similarities and differences observed through data collection during frequent debriefings with the thesis supervisor.

3. Confirmability. Confirmability in qualitative research is dependent on many factors such as the selection of participants, data collection and analysis methods and the ease for others to follow the audit trail (Sandelowski, 1986; Streubert \& Carpenter, 2011). A detailed audit trail outlining the activities of the study and the process that was followed was maintained in this study. These include but are not limited to observation and interview notes and original transcripts, details about the data collection methods and a clear outline of the data analysis process. A detailed coding table and coding tree that demonstrated a clear link between the data and the study findings was described. I also engaged in frequent dialogue with the thesis committee members throughout the data collection and data analysis process. The committee members were provided with a sample of 4-6 transcripts to confirm the codes and categories derived from the data during the early stages of the analysis. 
4. Transferability. Transferability refers to how well the findings from the study can be applied or transferred to other settings (Loiselle et al., 2011; Streubert \& Carpenter, 2011). The information provided must be sufficient so other researchers can make a judgement on the context transferability to a different setting (Graneheim \& Lundman, 2004; Loiselle et al., 2011). A thick description of the descriptive and demographic data of the setting, participants, and recruitment strategies and numbers of hours spent in the field was described (Loiselle et al., 2011). (See Chapter 4 Results).

\section{Summary}

This chapter provided a detailed account of the methodological approach and approach to rigor exploring the factors and processes affecting the OR-PACU handover. The results will be discussed in the following chapter (Chapter 4). 


\section{CHAPTER 4: RESULTS}

The results of the qualitative content analysis guided by the overarching question of what are the safety practices and the factors that affect the OR-PACU handover will be presented in this chapter. The chapter will begin with a summary of descriptive information of the participants and the observed handovers followed by the results from the qualitative data analysis results.

\section{Descriptive information: Participants}

This study used a convenience sampling for the recruitment of 72 surgical patients (Table 1.a) and 115 health care providers (HCPs) from OR and PACU nursing, anaesthesia and surgical teams (Table 1.b). A total of 58 OR-PACU handovers was observed (Table 2.a).

Details of the surgical patients recruited in this study are illustrated in Table 4.a). There were 92 patients in total who expressed interest in participating during the data collection period from June to August, 2015. For reasons outlined in the table, 81 out of the possible 92 patients were consented and 58 handovers were observed. Patients were consented one to two hours prior to their surgery. The time provided to recruit patients was brief. During this time patients were also engaged in the perioperative admitting process before their surgery, including the administration of pre-op medications or meeting and talking to various HCPs. These activities coincided with recruitment time and as the researcher had an obligation not to cause any delays, in 11 cases of the 92 potential handovers there was insufficient time to consent patients in the available timeframe.

Table 4.a) OR-PACU Handover Study: Participants Information: Patients 


\begin{tabular}{|l|l|}
\hline Patients willing to participate & 92 \\
\hline Patients consented & 81 \\
\hline Patients/handovers observed & 58 \\
\hline Patients interested but no time to consent & 10 \\
\hline Patients interested but did not sign a consent & 1 \\
\hline Patients informally interviewed beyond observation & 4 \\
\hline
\end{tabular}

Details of the health care professionals who participated in this study are illustrated in Table 4.b). Of the 51 anaesthesia providers 35 participants consented. This included staff anaesthesiologists, fellows, residents and anaesthesia assistants (AAs). There were 16 anaesthesia providers out of the total who were not consented. These were residents and fellows, and because their rotation was predominantly in the obstetric unit they were separate from the main ORs. Out of the potential 58 OR Registered Nurses (RNs), 48 consented and out of the potential 25 PACU RNs, 20 RNs consented. Four OR nurses and five PACU nurses were not consented because of working permanent weekends or evenings (times of the day not applicable to this study). Surgical providers were consented as needed prior to or after the handover provided they were participating during the handover report that was being observed. In total, 13 surgical team members consented to participate in this study.

Table 4.b) OR-PACU Handover Study: HCP Participants Information

\begin{tabular}{|l|l|}
\hline Anaesthesia Providers (staff, residents, fellows, assistants) & 35 \\
\hline Surgical Providers & 13 \\
\hline OR Nurses & 48 \\
\hline
\end{tabular}




\begin{tabular}{|l|l|}
\hline PACU Nurses & 20 \\
\hline
\end{tabular}

Details of the focus group session are illustrated in Table 4.c). Nine healthcare professionals participated in the focus group: anaesthesia $(n=2)$, surgical $(n=3)$, OR nursing $(n=3)$, and PACU nursing $(n=1)$. Recruiting additional PACU nurses was not possible due to the staffing shortage and scheduling conflicts. The researcher met with PACU staff on an individual basis for two days prior to the focus group session to respond to the same questions asked during the handover. This feedback was incorporated in the discussion during the focus group session by the researcher and was added also to the focus group data analysis.

Table 4.c) OR-PACU Handover Study: Recruitment Information Focus Group Session:

\begin{tabular}{|l|l|}
\hline Anaesthesia Providers (including anaesthesia assistants) & 2 \\
\hline Surgical Providers (including fellows) & 3 \\
\hline OR Nurses & 3 \\
\hline PACU Nurses & 1 \\
\hline
\end{tabular}

\section{Descriptive information: Handovers}

Details about the OR-PACU handovers are illustrated in Table 5.a). Overall 58 handovers were observed and 52 were included in the study. The initial six handovers were pilot observations during which the researcher developed strategies on how to best optimize the data captured during the observed handover. Of the 81 patients consented, 23 handovers were missed because of timing issues. These included reasons such as the case finishing after 5PM (6), patient being transferred to ICU instead of PACU (3), overlap of two handovers (3), missing a handover by the researcher (3), and recruiting another patient during a handover in progress (2). On five 
occasions when a single member of the team chose not to participate in the study the entire handover was not eligible for observation or data collection. No specific reason was provided for their decision not to participate in the handover observations.

Table 5.a) Descriptive Information on the OR-PACU Handovers in this study:

\begin{tabular}{|l|c|}
\hline Handovers observed (initial 6 as a pilot) & 58 \\
\hline Handovers included in the analysis & 52 \\
\hline Handovers missed due to case finishing too late & 6 \\
\hline Handovers missed due to team member did not consent to participate & 5 \\
\hline Handovers missed due to the patient being transferred to ICU & 3 \\
\hline Handovers missed due to researcher consenting another participant & 3 \\
\hline Handovers missed due to two or more handovers overlapped & 3 \\
\hline Handovers missed due to late arrival of the researcher to PACU & 3 \\
\hline
\end{tabular}

Breakdown of the observed handovers based on the admission status of the surgical patient and the type of surgical specialty is illustrated in Table 5.b) and Table 5.c). The majority of observed cases were patients who stayed in the hospital overnight post-operatively $(n=42)$. There were eight patients included who were discharged home on the same day of the surgery. There were also two patients who were in-patients in the hospital.

Table 5.b) Handover Type by Patient Admission Status

\begin{tabular}{|l|l|}
\hline \multicolumn{1}{|c|}{ Patient Admission Status: } & Number of Observed Handovers: \\
\hline Day Surgery (DS) & 8 \\
\hline
\end{tabular}




\begin{tabular}{|l|l|}
\hline Same Day Admit (SDA) & 42 \\
\hline Inpatient (IP) & 2 \\
\hline
\end{tabular}

Table 5.c) Handover Type by Surgical Specialty Type

\begin{tabular}{|l|l|}
\hline \multicolumn{1}{|c|}{ Surgical Specialty/Type: } & \multicolumn{1}{|c|}{ Number of Cases: } \\
\hline Orthopaedics (arthroplasty \& sarcoma) & 15 \\
\hline General surgery & 12 \\
\hline ENT & 8 \\
\hline Gynecology/Urogynecology & 6 \\
\hline Maxillo-facial & 5 \\
\hline Plastics & 4 \\
\hline Urology/Cysto & 2 \\
\hline
\end{tabular}

Details about the observations are illustrated in Table 5.d). Observations of the OR-

PACU handovers in this study took place over a two month period during June and July in 2015 between the hours of 07:30 and 17:00. There was approximately 22 cases scheduled for each day and the average number of observed handovers was four per day. The researcher spent 118 hours in the field (PACU) during the observation period. The time spent in the field included recruitment activities, informal interviews with participants and observations of the everyday flow of the unit. The total length of the handovers observed during the course of this study was six hours and 25 minutes. The longest observed handover in this study was 14 minutes and the shortest was two minutes. The average length of a handover was seven minutes. 
Table 5.d) Handover Timing Information:

\begin{tabular}{|l|l|}
\hline Handover observation timeline by date & June 12-July 24, 2015 \\
\hline Observation timeline by time of day & $7: 30-17: 00$ \\
\hline Total hours researcher spent in the field (PACU) & $118 \mathrm{~h}$. \\
\hline Total handover length & $375 \mathrm{~min} / 6.25 \mathrm{~h}$. \\
\hline Average length of a handover & $7.2 \mathrm{~min}$ \\
\hline The minimum length of a handover & $2 \mathrm{~min}$ \\
\hline The maximum length of a handover & $14 \mathrm{~min}$ \\
\hline Average number of cases scheduled per day during observation period & 22 \\
\hline Average handovers observed per day & 4 \\
\hline
\end{tabular}

\section{Descriptive findings: General information}

Details about the team members who accompanied the surgical patient during the transfer from the OR to the PACU and also the team members who actively participated during the ORPACU handover report are illustrated in Tables 6. a), b), and c). The findings from the observations show that anesthesia providers accompanied the surgical patient 100 percent of the time, whereas the surgical team members were present during the transfer 71 percent of time and the OR nurse only 65 percent of time. Participating during the handover 100 percent of the time were anaesthesia providers and OR nurses, and members of the surgical team participated 40 percent of the time. PACU nurses were participating during the handover at all times as recipients of the report. 
Table 6.a) Handover Participation Information for Anaesthesia Providers

\begin{tabular}{|c|c|c|c|c|c|}
\hline & \multicolumn{4}{|c|}{ Anaesthesia Providers } & \multirow{2}{*}{$\begin{array}{r}\text { Overall } \\
/ 100 \%\end{array}$} \\
\hline & $\begin{array}{l}\text { Staff } \\
152^{*}\end{array}$ & $\begin{array}{c}\text { Fellow } \\
152^{*}\end{array}$ & $\begin{array}{c}\text { Residents } \\
\text { /52* }\end{array}$ & $\begin{array}{l}\text { AAs } \\
152 *\end{array}$ & \\
\hline Accompanying the patient to PACU & 25 & 21 & 20 & 5 & $100 \%$ \\
\hline Participating in the handover & 23 & 17 & 14 & 4 & $100 \%$ \\
\hline
\end{tabular}

* during the 52 OR-PACU handovers observed

Table 6.b) Handover Participation Information for Surgical Providers

\begin{tabular}{|c|c|c|c|c|}
\hline & \multicolumn{3}{|c|}{ Surgical Providers } & \multirow{2}{*}{$\begin{array}{l}\text { Overall } \\
/ 100 \%\end{array}$} \\
\hline & $\begin{array}{l}\text { Staff } \\
152 *\end{array}$ & $\begin{array}{c}\text { Fellows } \\
152^{*}\end{array}$ & $\begin{array}{c}\text { Residents } \\
\text { /52* }\end{array}$ & \\
\hline Accompanying the patient to PACU & 10 & 17 & 19 & $71 \%$ \\
\hline Participating in the handover & 23 & 17 & 14 & $40 \%$ \\
\hline
\end{tabular}

* during the 52 OR-PACU handovers observed

Table 6.c) Handover Participation Information for Registered Nurses: OR and PACU

\begin{tabular}{|c|c|c|c|}
\hline & $\begin{array}{c}\text { Registered Nurses: } \\
\text { OR } \\
152^{*}\end{array}$ & $\begin{array}{c}\text { Registered Nurses: } \\
\text { PACU } \\
152^{*}\end{array}$ & $\begin{array}{l}\text { Overall } \\
/ 100 \%\end{array}$ \\
\hline Accompanying the patient to PACU & 34 & $\mathrm{n} / \mathrm{a}$ & $65 \%$ \\
\hline
\end{tabular}




\begin{tabular}{|l|l|l|l|}
\hline Participating in the handover & 52 & 52 & $100 \%$ \\
\hline
\end{tabular}

* during the 52 OR-PACU handovers observed

The observations revealed that the PACU nurse was available to receive the surgical patient 92 percent of the time, and 39 percent of the time there was more than one PACU nurse available to receive the patient (See: Table 7). The curtains for privacy were drawn 57 percent of time prior to the patient arriving to the PACU bay and during the handover. Overall patient privacy was maintained 95 percent of the time.

Table 7. PACU Nurses Availability and Privacy Considerations

\begin{tabular}{|l|c|}
\hline & Yes \\
& $152^{*}$ \\
\hline PACU nurse available & $48(92 \%)$ \\
\hline More than one PACU nurse available & $20(39 \%)$ \\
\hline Curtains drawn for privacy & $29(57 \%)$ \\
\hline Privacy somewhat maintained & $49(95 \%)$ \\
\hline
\end{tabular}

* during the 52 OR-PACU handovers observed

The data analysis focused on what information was communicated during the handover report and by whom and the information communicated was summarized on a data collection sheet. The information communicated most often during the handover was the patient name, age, allergies, medical history, type of procedure, fluid management and pain medications given pre and intraoperatively. The items reported most frequently were also the items that overlapped by both the anaesthesia provider and the OR nurse. Items reported the least were postoperative care and discharge plan, intraoperative positioning, local anaesthetic administration in the OR, skin 
integrity and who will communicate with the family postoperatively. Further details about the intraoperative phase and how that may affect the postoperative care were also rarely discussed during the handover.

\section{Results}

The qualitative content data analysis identified four main categories (ownership, interruptions and distractions, transfer of information and workflow) each of which encompassed two to three generic categories and a number of sub-categories (See: Figure 1.). The selected quotes used in this chapter are from individual participants and were chosen to capture the essence of the category.

Figure 1.) Coding Tree: Exploring OR-PACU Handovers Study: Data Analysis Results

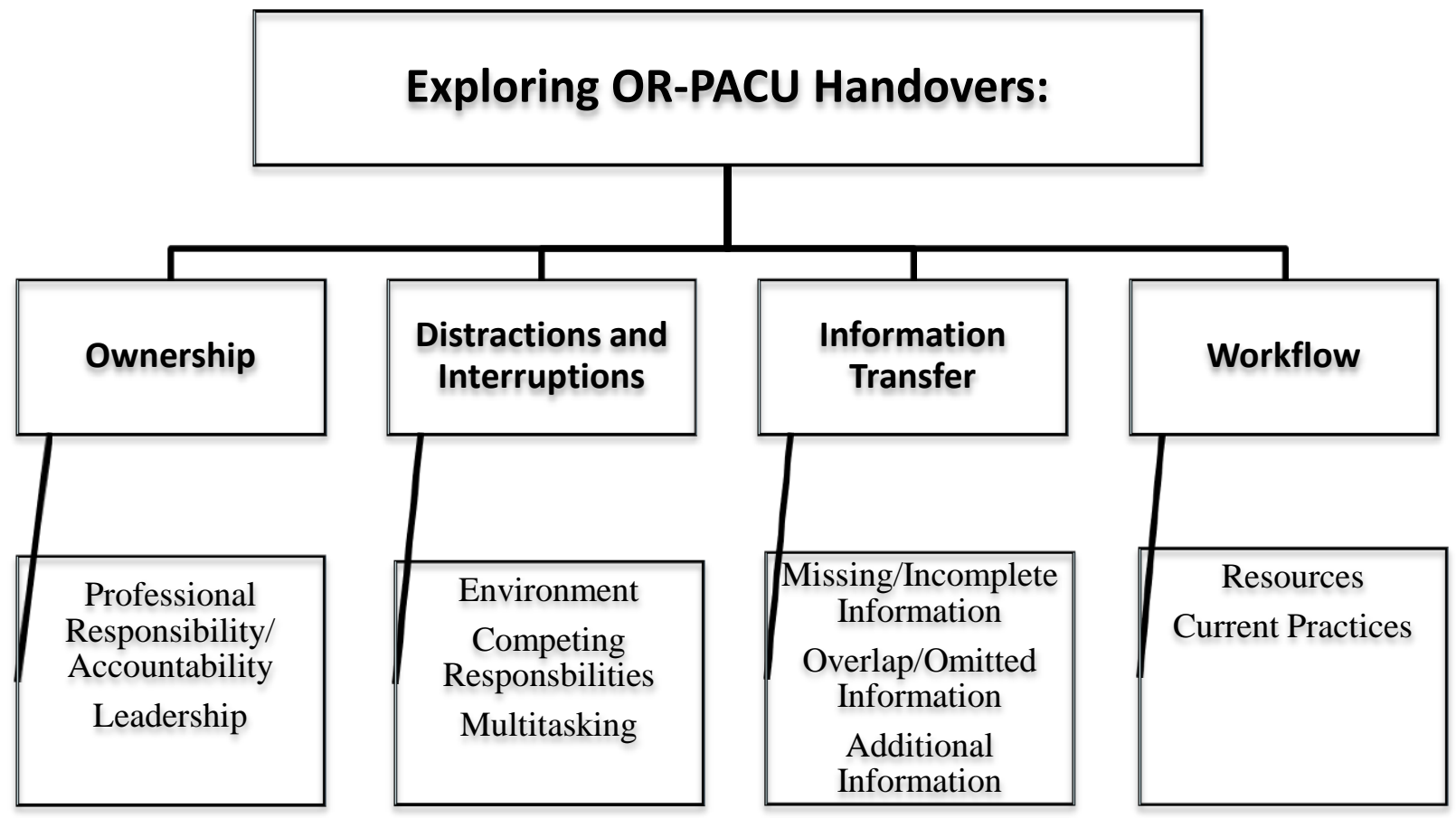

\section{Ownership}


Ownership as a main category was characterized as individual or team actions and behaviors of the individual profession. There were two generic categories defining ownership: 1) Professional responsibility and accountability and 2) Leadership (Figure 1.a).

Figure 1.a) Exploring OR-PACU Handovers: Main Category: Ownership

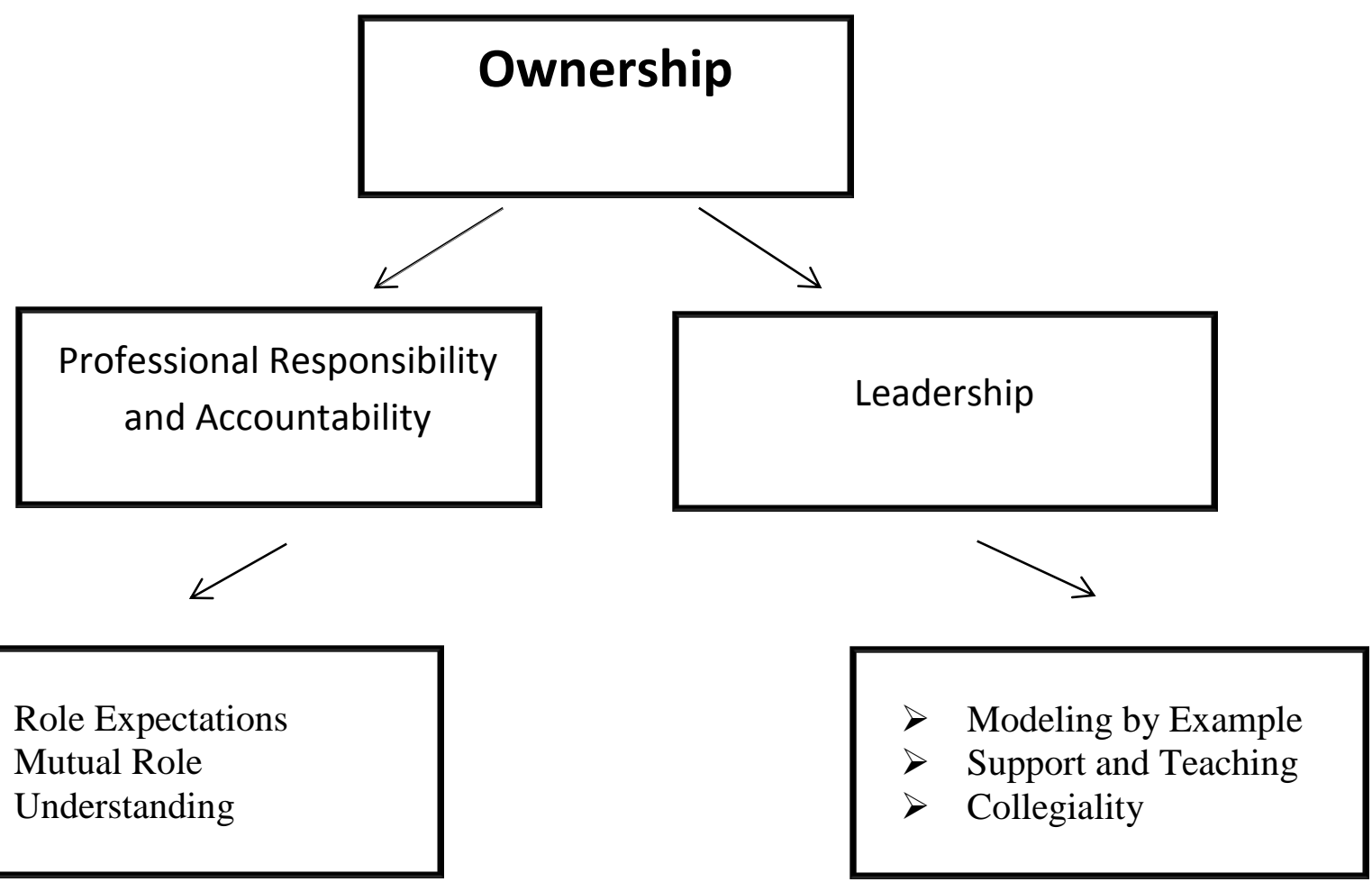

Professional responsibility and accountability. Professional responsibility and accountability were defined as the responsibility and accountability of the individual for decisions, actions and consequences during the handover. The codes in this category were grouped under the following sub-categories: role expectations and mutual role understanding. Role expectations were not clear amongst team members, specifically surgical residents and fellows who were often not aware they should be actively participating in the postoperative handover. As one HCP stated: 
"Yes, I believe that someone from the surgical team should be there (during the handover) everytime. I don't know why they are not. I don't even think they know they should be there"

-surgeon \#1

The surgeon or the members of the surgical team (fellows or residents) participated during the handover on a regular basis only when the surgical procedure directly affected the postoperative care of the patient. For example, when there was a specific positioning or monitoring of the patient required in PACU such as a flap and pulse monitoring, oral care or airway management or particular limb positioning. Surgical team members (residents and fellows) who were early in the rotation often asked the PACU staff for directions and clarification of their role. In contrast, junior anaesthesia providers were guided very closely by senior anaesthesia staff on what the expectations of their roles were during the handover process. Prioritizing the patient upon arrival as opposed to reporting before the patient was settled was also identified as being a factor affecting the handover. As stated by one HCP participant: "Some nurses start giving report as soon as they walk through the PACU doors and it is like "Hold on, hold on!!"... and then there are nurses who come in and help you to check the patient in and put the monitors on first before the report and this is great! This shows that the patient is the priority"

-PACU nurse \#1

Mutual understanding between the HCPs about their expectations and their role was found to be necessary for effective communication. For example, providing the anaesthesia providers with details of the scope of practice of the $\mathrm{RN}$ helped them understand why their orders needed to be written specifically, such as central line or nasogastric tube placement conformation status. Understanding why and how intraoperative charting was being done in a certain way was also discussed by the HCP participants. Similarly, mutual understanding of the 
daily responsibilities and workload amongst the HCPs also provided clarity of expectations. As one HCP participant stated:

"I understand that you (the OR nurses) have to return and do other cases, so I guess it would be helpful to know what you do and why you are rushing out of here... not everyone knows how you work and not everyone understands."

-PACU nurse \#2

Other subcategories under the role expectation category were related to professional practice and expectations. For example demonstrating respect to colleagues, patients and their families, or maintaining patient privacy and confidentiality during the report by drawing the curtains and maintaining an appropriate voice level. Other examples included individual name and role introduction prior to the handover, following institutional policies, such as signing the nursing transfer sheet as part of the required paper documentation, or reporting on all of the items as per existing transfer documents:

"Some nurses don't even bother telling you what is on the blue sheet (nursing transfer sheet), they just bring it and it is up to us to get the information from it ... I have never had to sign the blue sheet and no one from the OR ever asked me to ... and this is strange because I will always have the nurses up on the floor sign to make sure they acknowledge my report"

-PACU nurse \#3

Leadership. Leadership was identified as guidance or direction that was provided to the participants of the handover report. The codes under this category were grouped into three subcategories: modeling by example, support and teaching. Lack of modeling and direction from senior surgical staff were identified as one of the potential reasons for the inconsistencies in the surgical team member's involvement in handovers. Senior surgical providers acknowledged that participation of the surgical team members in the handover was not necessarily included in the 
curriculum and was not part of their residency or fellowship training. One HCP participant stated:

"I think it is important having someone (the surgical staff) to role model this (participation during handover) so that it becomes engrained into the team members role and it is understood that it will not be just OK to finish up case ... you get to the PACU after the case and it just becomes a new norm"

-surgical provider \#2

During the first period of data collection there was a changeover in the anaesthesia and surgical residents and clinical fellows' rotation. Throughout this phase, support and teaching was evident in all situations where both the senior and junior staff were present during the handover. This was applicable especially to anaesthesia providers who were always supportive and took extra time to teach the new residents and fellows. The staff often provided guidance, examples and the rationale of what was to be reported during the handover. Support was also evident by anaesthesia providers remaining in PACU after the handover report until the patient is completely settled in. PACU nurses helped each other consistently despite their own patient responsibilities. PACU nurses and other HCPs also often provided extra help after patient arrival to PACU; this included settling the patient, moving the patient up the bed or to a hospital bed.

In summary, ownership as a main category was characterized by professional responsibility and accountability and leadership. The emerging subcategories included role expectations, role modeling, teaching and support and evolved around the notion of mutual understanding of individual roles and expectations, modeling by example, collegiality and support during the handover.

\section{Distractions and interruptions}


The main category of distraction and interruptions was defined by any factors and processes that affected the attention of the handover participants or their activities during the handover report. Any factors in the environment that required the participants of the handover to stop the report or activities related to the handover, such as attending to a different patient or answering a phone during the report, were classified as interruptions. Any factors in the environment that affected the attention of the handover participants, such as noise or overhead announcements as noted by visual cues, for example a facial expressions, were classified as distractions. There were three underlying generic categories that defined distractions and interruptions: 1) Environment, 2) Competing responsibilities and 3) Multitasking (Figure 1.b).

Figure 1.b) Exploring OR-PACU Handovers: Main Category: Distractions and Interruptions

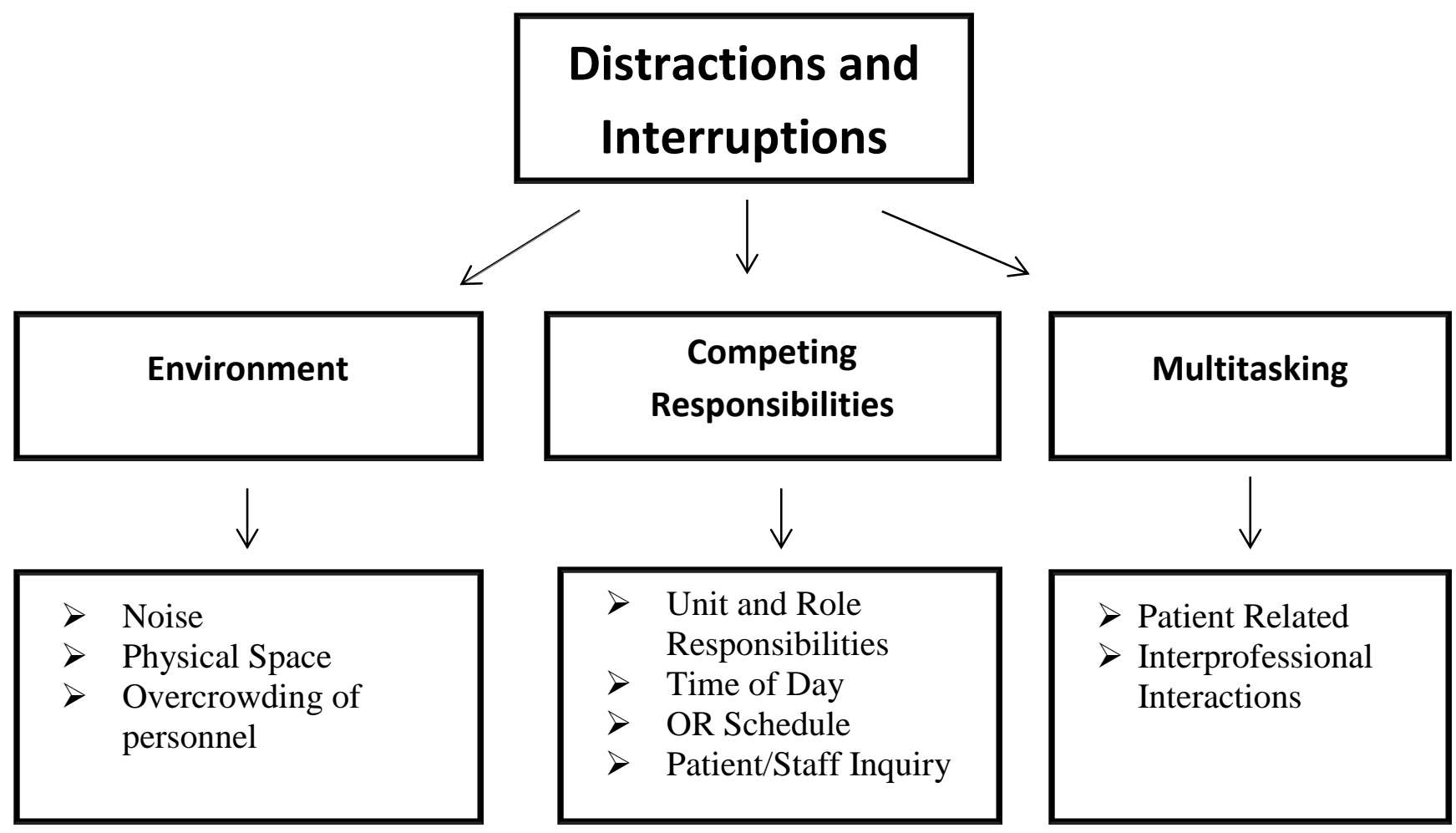


Environment. Environmental factors were identified as factors and processes that affected the physical space in which the handover took place. The codes in this category were grouped under the following sub-categories: noise, space and personnel. These were for example noise from telephones, pagers, furniture location and equipment availability and patients, staff or visitors flow throughout the PACU. It was not uncommon to have tables, chairs and other equipment obstructing the transfer of the surgical patient which required the HCPs to clear the area in order to progress to the final destination of the bed bay. Missing monitoring equipment or a part of the monitors was also a frequent issue and delayed or affected the immediate patient care.

It was not uncommon to have the PACU department overcrowded with HCPs from various teams and individual professions related to the perioperative setting and other specialties such as cardiac or social work. Having a PACU overcrowded with personnel was especially evident in the afternoon hours and at 3 PM close to the end of the day shift. This affected the movement of incoming and outgoing patients throughout the unit in an already crowded space. It also increased the noise level and affected the privacy and confidentiality during the handover. It was often observed that patients were clustered together despite the space availability of patient bays throughout the unit. This was to allow the PACU nurses to cross-cover two patients or to be able to help each other. Moreover, the patient bays are small and close to each other which caused further congestion around the patient who was being settled.

Lack of physical space often created a "code R" situation during which the PACU was unable to accept patients ready to be transferred from the OR. The OR staff were concerned of not having a spot in the PACU after surgery and often arrived at the PACU with the patient unannounced or called for a patient bay to the PACU before being ready to leave the OR. This 
led to issues in the PACU with the patient flow, as demonstrated by the following quote from one PACU nurse:

"The OR needs to call us when they are ready to come to us [the PACU], not when they decide to come or when they are still not ready for another half hour. We are not a hotel; you can't book in advance or check in without at least calling in first."

-PACU nurse \#4

Competing responsibilities. Competing responsibilities were seen as any situation related to role and unit responsibilities requiring immediate attention from the participants during the handover. The codes in this category were grouped under the following sub-categories: unit and role responsibilities, time of day, OR list schedule, patient and staff inquiry. For PACU nurses competing responsibilities included in-charge and coordinating duties as well as having a responsibility over a patient at the same time. This was demonstrated by the following quote:

"This place can be really crazy, sometimes I have to be in charge and talk to anaesthesia and surgery, answer the calls, take the patient that comes in ..... I have people yelling and coming and going and I can't concentrate on one thing."

-PACU nurse \#5

Competing responsibilities were also experienced by the OR nurses based on all the demands associated with their role. For example, end of case responsibilities as illustrated by the following quote from an OR nurse:

"The end (of the OR case) is so busy: you have to go out with the patient, call for housekeeping, you have to print, you have to give report, you have to run back to set up for the next case or check the next patient. It is hard sometimes to choose what to do first."

-OR nurse \#1

Competing responsibilities were also related to cross-covering patients in situations where the PACU nurse had to care for more than one patient and both required attention at the 
same time. Similarly, OR nurses and anaesthesia providers felt pressured to hurry the handover and to attend to the next patient or get the next case organized and going. This was closely aligned with the OR list schedule for the day and is demonstrated by the following quote from an OR nurse:

"I need to go first [during the handover report]. I can't stay here [in PACU] and listen to the whole report, I have to go. We have 2 more cases to do and there is no relief."

-OR nurse \#2

The above quote also demonstrates competing responsibilities related to personal priorities and interests. For example, participants were often worried about running late; not having relief and the threat of having to stay to complete the case which took priority over providing the handover report. The participation of the staff and the length of the handover was also closely related to the time of day. The handover for the last case of the day, especially when close to the end of the shift, was faster and there was more competition between the participants of who would report first.

Multitasking. Multitasking during handover report was predominantly patient related and involved tasks surrounding patient care. The codes in this category were grouped under the following sub-categories: patient related and interpersonal interactions. For example, it was common to place the monitors on the patients upon arrival to PACU while also listening to the report. It was also common to engage in activities such as checking the computer orders, skin condition, wound, dressing, drains and the Foley catheter while listening to the report. Other activities such as drawing up medication or charting were also frequently observed and frustration about multitasking was felt from some of the participants as demonstrated by a quote from one anaesthesia provider: 
"I can't understand why people wouldn't just wait for the patient to get settled in the $P A C U)$ and then get everyone's attention during the handover report. Instead everything happens at once!"

-anaesthesia provider \#1

Out of the 52 handovers there was only two during which the participants stopped all activities around the patient and fully engaged in the handover report. This was accomplished because the providers of the report waited for the patient to be settled first before starting the report. Having the opportunity to settle the patient first was appreciated by the PACU nurses as evident in the following quote:

"We normally need to divide our attention between the patient and listen to the report ... we need to have time to do our focused assessment prior to the report so we can concentrate on what they (the participants of the report) are saying"

-PACU nurse \#6

Additionally, personal conversations and interactions contributed to multitasking. For example, it was common for two participants to be engaged in a side conversation while listening to another participant provide the report. This was highlighted in a quote from one anaesthesia provider:

"Oh it would be beautiful if everyone was just quiet, listened to you and made notes. There should be silence when someone talks"

-anaesthesia provider \#2

In summary, distractions and interruptions as a main category were characterized as physical environment, competing responsibilities and multitasking. The emerging subcategories included noise and physical space, overcrowding of personnel, unit and role responsibilities, time of day and the OR schedule, patient and staff inquiry, and patient and individual priorities. The 
subcategories evolved around factors that affected the attention of the participants during the handover.

\section{Transfer of information}

The main category titled transfer of information was defined as any communication between the HCPs during the handover in which necessary information was provided for delivering safe and efficient care for the surgical patient. There were three underlying generic categories that defined transfer of information: 1) Missing and incomplete information, 2)

Overlap and repeat of information and 3) Additional information (Figure 1.c).

Figure 1.c) Exploring OR-PACU Handovers: Main Category: Transfer of Information

\section{Transfer of Information}
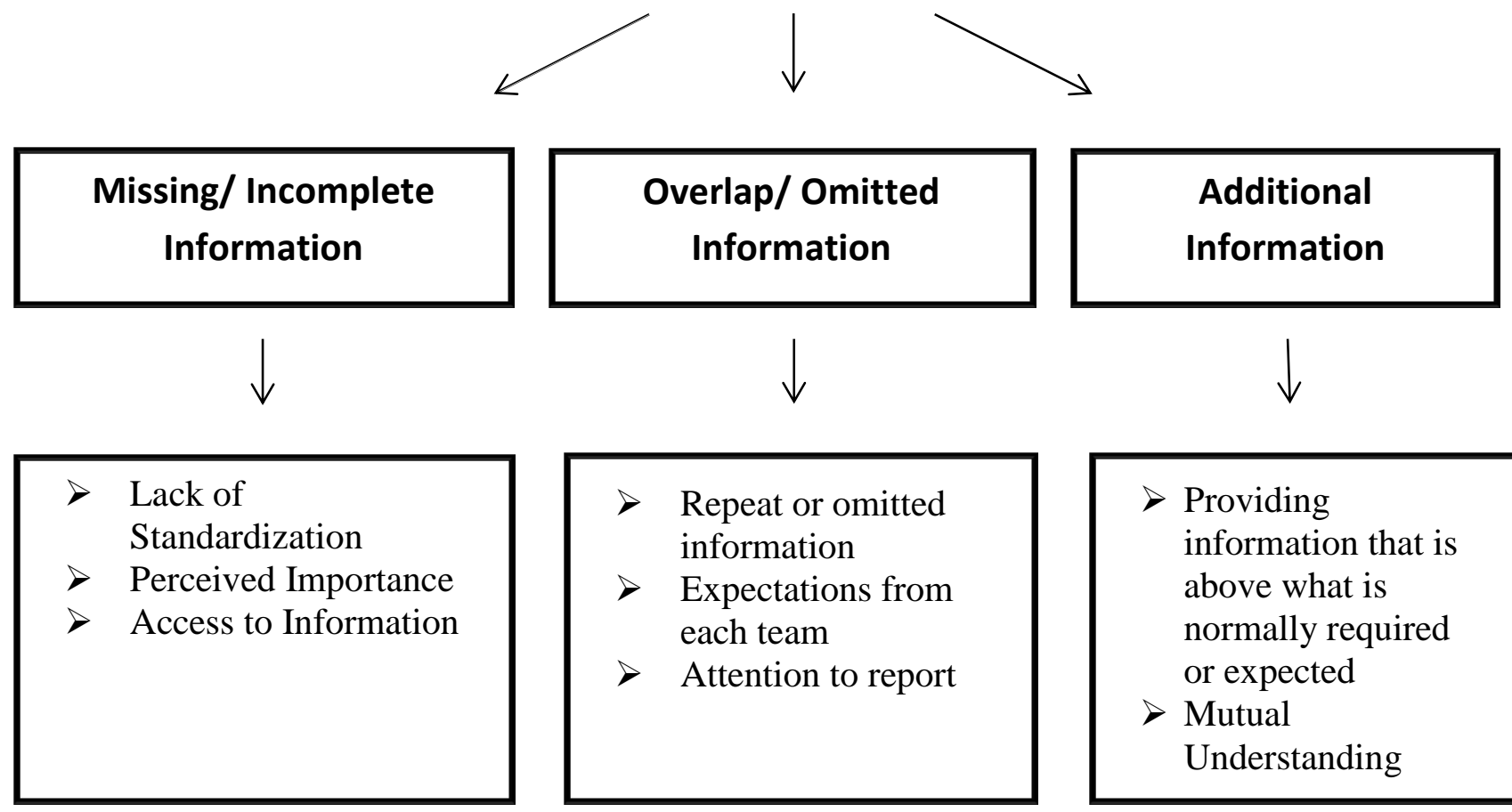

Lack of

Standardization

$>$ Perceived Importance

$>$ Access to Information

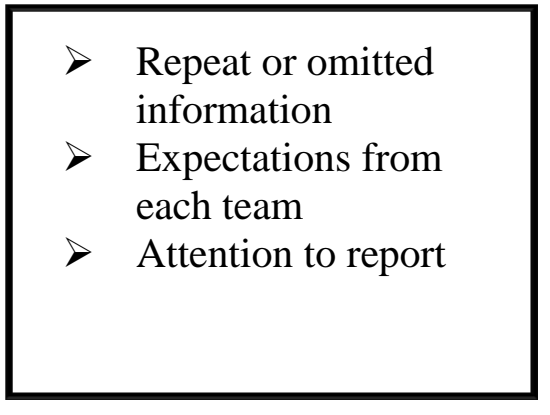

Providing information that is above what is normally required or expected Mutual Understanding 
Missing and incomplete information. Missing and incomplete information was defined as any information that was missed or was not communicated completely during the handover report. For example reporting on the Foley catheter in situ but not reporting on urine output was considered as an incomplete information transfer, and failing to mention that there was a Foley catheter in situ and the urine output was considered as missed information. The codes under this category were grouped into three sub-categories: lack of standardization, perceived importance and access to information. Missing and incomplete information often resulted from lack of a standardized process during the handover. Many participants suggested standardization to avoid missed or incomplete information when asked what would improve the handover:

"What would make the handover better? It would help to have a standardized process and something easy to use"

-anaesthesia provider \#3

"It would be better if they (the handover participants) use a systematic approach and some of them do have their own system."

-PACU nurse \#7

Lack of standardization was evident by the variance in the information provided by the team members during the handover report. During handovers, anesthesia providers relied on the anaesthetic sheet for information and OR nurses relied on the nursing transfer sheet. Items reported most inconsistently were: intra-op positioning, skin condition, local administration, surgical procedure information (what was done and what is expected), pre-op pain threshold, pain control post-op, discharge planning, dementia-mental status pre-op, EEG, blood work and vital signs information, lines placement and confirmation, intra-op events, family whereabouts 
and communication post-op, and patient specific information. Lack of standardization was also linked to perceived importance of information. The information reported was often dependent on what the individual perceived as important or necessary to be included in the report despite existing information sheets that were commonly used during the handover. As demonstrated in the following quotes:

"We normally use the anaesthetic sheet to report from and I would report on what I think is necessary for the patient from it"

-anaesthesia provider \#4

"It would be nice to know what each team is reporting. I have no idea what anaesthesia is saying and what they should say, so I just go and say what I think is important."

-OR nurse \#3

"The information provided is basically dependent on the anaesthetist and what they think."

-anaesthesia provider \#5

Further issues related to missing or incomplete information were associated with having access to information. Frequently the patient chart did not accompany the patient from the OR. This was often due to the surgical team members holding on to the chart for charting purposes, the OR team members forgetting to bring the chart with the patient from the OR, or the chart coming with the patient but hidden under the mattress. Not having the chart available was an ongoing issue as demonstrated by the following quote:

"Patients now are more so then ever coming out of the OR without their chart and this is a concern because we (in PACU) can't accept them and we shouldn't take the report without the chart ... would you (the OR nurses) take the patient to the OR without the chart? I doubt it! So why do you expect we would take the patient without the chart? 
Having access to information was also evident by the presence of the team member or the whole team to either provide the information or to be available and accessible for questions. It was further observed that it was not necessarily the presence of the team member or the team members, but rather their active involvement during the report or their approachability. For example, despite $71 \%$ of surgical members being present in PACU during the handover, only $40 \%$ were actively participating during the handover report. The surgical team members would come after the handover report was completed and all other team members had left the patient. Surgical team members were also often found to come into PACU with the patient and then slowly move away to either listen to the handover report or to write the patient orders in the chart or the nearby computer, instead of being active participants during the report.

"The surgeons should be there to provide the information...we don't know what they want post-op"

-anaesthesia provider \#6

"Having surgeons around would eliminate the phone calls to them from us to gather info"

-PACU nurse \#9

In contrast to not having the team members present and participating, having too many team members present led to information overload or repeat of the same information by various team members. This led to the recipient not hearing the report and informing the next generic category titled overlap and omitted information.

Overlap and omitted information. Overlap of information was identified as any information that was communicated by more than one profession. However, there were also 
instances where professionals believed that information was already communicated and to avoid redundancy did not communicate the necessary information. This was identified as omitted information. The codes under this category were grouped under three sub-categories: information overload and omission, expectations of each team member and type of information. Most frequently the overlap of information happened between the anaesthesia provider and the OR nurse. The items overlapping the most during the handover report were allergies $(65 \%)$, patient name $(52 \%)$, procedure $(44 \%)$, patient age (28\%). Overlap of information was closely tied to omitting items that needed to be reported. Participants suggested that there was a lack of direction as to what items should be reported by what team member. As such participants often opted not to report on items they assumed would be reported by other teams. Participants also often suggested there was an overlap during the report:

"Redundancy. Redundancy of the information is really painful"

-OR nurse \#4

"Sometimes I just like to fill in the blanks and say what I think is important not to repeat what anaesthesia said and then I'm accused of giving out bad report"

-OR nurse \#5

Overlap and omitted information were tied closely to understanding the expectations of what is required during the handover from each team member. Participants were not clear as to what information should be reported by them and what information should be reported by other team members. They were often unclear of what information the PACU nurses expected or needed during the handover in general:

"It would be really nice to know what each team is reporting" 
"Expectations, what do you expect from me? What do you want me to say?"

-anaesthesia provider \#7

"For example they tell you the local and the strength and how many mls. Well I don't really need to know all that, all I want to hear is there is a local and when it was given... but then again I don't think they know that"

-PACU nurse \#10

Overlap of information often led to anticipated expectations of the report and to diminished attention to the report from the receiver:

"By now I know what I will get from people and what info they will provide during handover. With some people its like "why do you even bother"? You are not saying anything anaesthesia didn't already say ... I know I don't have to listen I just tune out and concentrate on what I'm doing",

-PACU nurse \#11

Furthermore on many occasions the information communicated by the OR team was irrelevant or insufficient to provide safe postoperative care. The information found to be important by the PACU nurses was missing and will be discussed under the generic category titled additional information:

“The OR nurses always report on the size of the Foley ... but I (the PACU nurse) don't really care about the size of the Foley. It is irrelevant to me ... unless you say the size of the Foley is this and it is important because of that .... but that rarely ever happens."

-PACU nurse \#12 
Additional information. Additional information was identified as any information that was above what is normally reported as per the handover sheets used (anaesthesia record and nursing transfer sheet). The codes in this category were grouped under the following subcategories: additional information and mutual understanding between HCPs of what information is important. Mutual understanding was closely tied with knowing the expectations of what and how much should be reported. It was often not enough to report on all the items on the above mentioned transfer sheet. At times it was necessary to provide additional information that would enhance the recipients knowledge about the patient. For example this would include additional information on the patient's mental status and mood pre-op, patient mobility issues, any personal wishes discussed with the patient in the pre-op period, or intra-op events that affected the normally expected outcome. For example, it was often communicated that the patient has been taking opioids pre-op but it was not further discussed what their normal threshold is for pain and pain medications:

"Pain management post op, especially if the patient has a chronic issue and then we don't know that ... and then the pain meds aren't working ... we need to know the threshold for that patient and we don 't'"

-PACU nurse \#13

HCP participants often mentioned the need to discuss the patient preoperative and intraoperative vital signs and what the normal or acceptable values were for the patient in the post-op period:

"It is very helpful when they go over the vitals and the numbers, especially if out of the ordinary. For example, what was the BP intra-op and what is tolerated now for this patient. Often we don't get that reported and then we have to guess or fish in the chart or call the team" 
A major component missing during the handover report was the surgical information or information related to the intraoperative period and the postoperative expectations. In particular, details about the surgery, incision and the procedure in the operating room.

"We need to know the surgical info, such as if there is a drain and then there is a lot of fluid coming out: is that normal? Like the other day, the patient came out and there was so much fluid coming out of the drain and I had to call the surgeon and then they said it was normal because they used so much fluid in the OR. It would be helpful to know that during the report ... I didn't have to call anyone ... or another example is like the blood on the dressing, if there is a lot of blood coming through and I don't know if that is normal or not."

-PACU nurse \#15

Among the information that was the least expanded on during the report were the expectations, for example: is this normal for this patient and under what circumstances should the team be called, i.e. haemoglobin levels or vital signs. Other information such as who will be taking over the patient post-op medically or whether the patient requires acute pain service were often not fully discussed. Furthermore, patient specific information was often not communicated completely. For example, on two occasions it was reported during the handover that the patient does wear glasses but no one further elaborated on the patient's inability to see without them. Other patient specific information such as, severe anxiety in the preoperative period was also often underreported. Likewise, information about the patient's family and whereabouts were only partially reported.

In summary, transfer of information as a main category was characterized as missing/incomplete information, overlap/omitted information, and additional information. The emerging subcategories included perceived importance, access to information, lack of standardization, repeated or omitted information, expectations from each team, and providing 
information above of what is normally required. The subcategories evolved around factors that affected the attention of the participants during the handover.

\section{Workflow}

Workflow as a main category was defined as factors and processes that affect the working environment as related to the OR-PACU handover. There were two underlying generic categories that defined workflow: 1) Resources and 2) Local practices (Figure 1.d).

Figure 1.d) Exploring OR-PACU Handovers: Main Category: Workflow

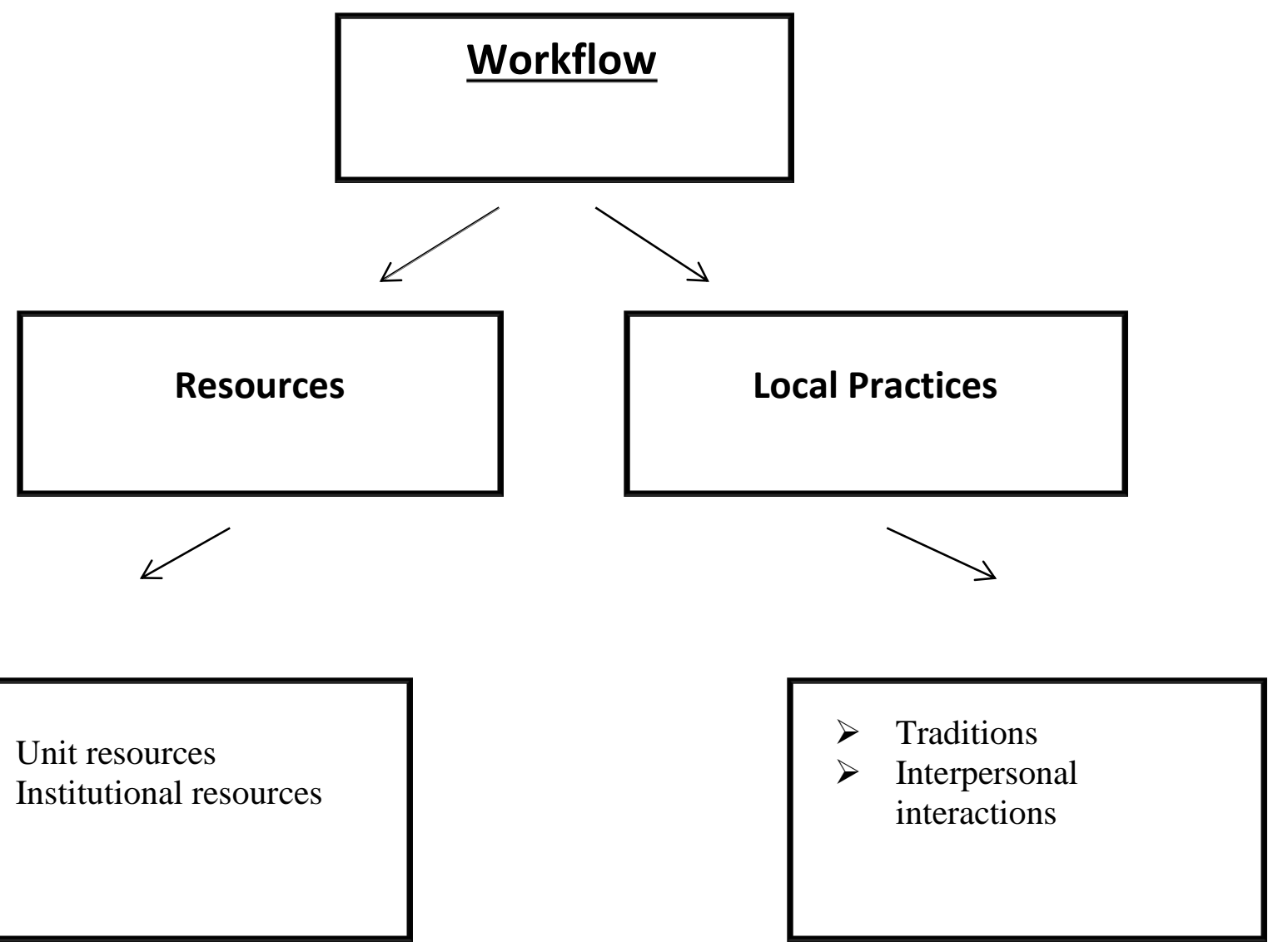


Resources. Resources were identified as existing unit or organizational resources that affect the perioperative environment. The codes under this category were grouped into two subcategories: unit resources and institutional resources. The unit resources included vital sign monitors, staffing coverage and space or bed availability for the surgical patient. For example, each time the patient arrived to the PACU they had to be attached to the blood pressure, ECG and pulse oximeter monitors. This took priority over the report which was usually happening congruently. Not having monitors that could remain attached on the patient from the OR and reconnected upon arrival to PACU was commented on by many HCP participants:

"Ideally the monitors used in the OR will just stay on the patient and then fit into the machines in PACU”,

-anaesthesia provider \#8

Lack of readily available resources such as patient beds were also illustrated in the following quote:

"The bed is not there when we need it (at the end of the case) so we can't transfer the patient onto it, then we get the patient on the stretcher and PACU gets upset because the patient is not on a bed ... or the bed comes as we are passing by the OR desk and then it follows us to PACU where it is really difficult to move the patient when PACU is full"

-OR nurse \#17

At times the PACU unit was short of staff which affected the patient/nurse ratio or led to "code R" which meant that the unit was closed to new patients. Other contributing factors to “Code R" were, for example, the surgical floor not having available space to move the patient from the PACU. As such unit related resources were tied closely to institutional resources. For example, although staffing shortage or backlog of in-patients in PACU was considered under unit resources it was often institutional resources that affected the unit flow. For example, 
participants perceived the lack of available bed space on surgical units to be related to the overall patient volume in the hospital and a chronic shortage of patient beds.

Another issue was related to required paper documentation that needed to be completed prior the patient leaving the OR. For example, OR nurses often reported they were unable to transfer the patient to PACU or be present for the full report because they were delayed by computerized charting and printing. This was illustrated in the following quote:

"Computers in the OR are a pain, really a big pain. We have to wait for 5 minutes to print ... then I get to PACU and I don't know what was said during the report or I'm not even there!"

-OR nurse \#7

OR nurses were required to print an intraoperative record before leaving the OR and at times there was a delay of 2-5 minutes between the time the record was competed to the time of printing the record. The OR nurses (35\%) often did not accompany the patient during the transfer to PACU because of waiting in the OR to print the record. This delay is highlighted by the following quote:

"It is not my fault that I am late (for transferring the patient to PACU and providing the report) I was waiting for the printout (of the intra-op report)."

-OR nurse \#8

The other day I waited for 10 minutes to even load the (computer) screen to get to the part where you click off to print."

-OR nurse \#9

Local practices. Local practices were defined as observed behaviors and common practices within the PACU. The codes in this category were grouped under the following sub- 
categories: traditions and interpersonal interactions. Traditionally, the handover report was verbal and happened when the PACU nurse was settling the patient upon arrival to the PACU. As such the nurse was only listening to the report without looking at the patient's chart or making notes about the report:

"I am a visual learner and I'm having difficulties when I'm getting report and it's all just verbal. I wish I could see the anaesthetic record during the handover to see what they have written and what information is on it. When I just get a verbal report it all sound like blalalalala to me ... I can't process that. I need to see the history; especially when it is extensive and I need to write it down and I would like to go over it with the team."

-PACU nurse \#4

The importance of having the chart available and having legible writing was also highlighted by the following quote:

"We need to have the chart in front of us when we get report and we need to be able to read what it says. Especially when it's a busy time and you have a back to back patients and then nurses don't want to take patients because they didn't even complete or see the chart on the first patient"

-PACU nurse \#6

In general, PACU nurses maintained eye contact with the participants of the handover as acknowledgment of listening to the report. They remained silent during the report and would not necessarily ask questions from the handover participants but rather opted for finding the information they needed in the chart after the handover report. This was supported in the following quote:

"In general we don't like to interrupt them (the anaesthesia providers) when they give report, because I don't want them to lose their train of thought. It is better if they use a systematic approach and some of them do have their own system ... and it is often easier for me to find the info in the chart after" 
In general, there was no particular order between who reports first during the handover, although the majority of OR nurses gave report first and left the PACU before the handover report was over. Further, there was usually no discussion between the anaesthesia provider and the OR nurse on who would report first. Typically, the team members did not introduce themselves by name and role upon arrival to PACU. Similarly, PACU nurses only introduced themselves to the receiving patient upon arrival to PACU by name and role occasionally.

Interprofessional interactions were evident in instances where HCPs who were friendly towards each other were more likely to interact more before, during and after the handover report. Participants felt more positive about the handover when they knew each other well and found that individuals who were more outspoken asked more questions during the handover report.

In summary, workflow as a main category was characterized as resources and local practices. The emerging subcategories included unit and organizational resources, traditions, local context, and interpersonal interactions. The subcategories evolved around factors that affected the flow in the unit and organization and directly or indirectly affected the handover.

\section{Summary}

This chapter provided a detailed description of the results from this study exploring the factors and processes affecting the OR-PACU handover. Informed by data collected from observations, informal interviews and a focus group session four main categories were identified during the data analysis process: Ownership, Distractions and Interruptions, Transfer of 
Information and Workflow. Each category was supported by two or three generic categories. Workflow was underpinned by professional responsibility and accountability, leadership and further by role expectations and mutual understanding, modelling by example, support and teaching. The category distractions and interruptions was supported by environment, completing responsibilities, multitasking and further by noise and physical space, unit and role tasks, time of day, the OR schedule, patient and staff inquiry, and patient and unit related activities completed at the same time. The category transfer of information was informed by missing and incomplete information, overlap and omitted information, additional information and further by lack of standardization, perceived importance, access to information, repeat or omitted information, expectations from each team, attention to report, and providing information that is above what is normally required. The category workflow was underpinned by resources and local practices and further by unit and institutional resources, traditions and interpersonal interactions. The findings will be interpreted in Chapter 5 Discussion and Implications. 


\section{CHAPTER 5: DISCUSSION AND IMPLICATIONS}

This study used qualitative descriptive methodology to explore the factors and processes affecting the OR-PACU handover. Insights were gained into the safety practices and work dynamics in the PACU environment towards informing the future design and implementation of a standardized team handover tool. The Theory of Collective Competence (Boreham, 2004) will be used to elucidate the results of this study, ownership, distractions and interruptions, information transfer, and workflow identified during the data analysis process. The study limitations, implications and future recommendations for practice, education, policy and research will also be reviewed.

\section{Theoretical framework}

The Theory of Collective Competence (Boreham, 2004) highlights the need for a collective and competent approach to teamwork and in relation to this study specifically ORPACU team handovers. The OR-PACU handovers involve members of various professions coming together as a unified team with a shared goal: safe management of the surgical patient. The results of this study are well informed within the three components of collective competence: 1) making collective sense of events in the workplace, 2) developing and using a collective knowledge base, and 3) developing a sense of interdependency (Boreham, 2004).

There are many factors that shape collective competence. In this study, the category of Ownership is characterized as individual or team actions and behaviors of the individual profession. The theory provided an understanding of how individuals make collective sense of their roles and professional responsibilities. Individuals possess knowledge of their own roles and responsibilities and when engaged in teamwork have a collective sense of understanding of 
the impact these actions and responsibilities have on patient care (Boreham, 2004; Manser et al., 2013). Teamwork requires effective communication which according to Boreham (2004) depends on the team's ability to overcome individual differences and backgrounds. Team competence is also dependent on shared experiences and the ability of the team to utilize these experiences to respond to situations appropriately (Hodges \& Lingard, 2012).

The category of Transfer of information was defined in this study as any communication between the HCPs during the handover in which necessary information was provided for delivering safe and efficient care for the surgical patient. The category Distractions and Interruptions were defined in this study as any factors and processes that affected the attention of the handover participants or their activities during the handover report. Workflow was defined in this study as factors and processes that affect the working environment and the completion of the OR-PACU handover. The theory elucidated results of this study related to communication practices and information transfer during the handover and consideration of the perspectives of organizational and social influences. The Theory of Collective Competence suggests common practices, relationships, social interactions and the environment all influence how individuals behave in a team environment and informed all the results of this study (Boreham, 2004; Hodges \& Lingard, 2012).

Understanding the handover process from the perspective of collective competence suggests approaching the results not from an individual, but rather from an organizational and social standpoint. The use of the theory further supports the development of strategies to support effective communication to address the similarities and differences in how individuals function as a team, for example during a team handover tool or process. 


\section{Discussion}

In this study OR-PACU handovers were observed from a variety of surgical services with perioperative staff from OR and PACU nursing, anaesthesia and surgical specialties. This section will discuss the findings of the four major themes identified during the data analysis process: ownership, distractions and interruptions, information transfer, and workflow informed by the existing literature.

\section{Ownership}

The professional responsibility and accountability of health care professionals to actively participate during transfer of care transitions was observed throughout the study. However, there was variation as to who was actively participating during the OR-PACU handover. Due to the nature of the surgical patient status in the immediate postoperative period, anaesthesia providers were very involved with the patient care and the OR-PACU handover. Perioperative nurses were also involved, as required by the OR transfer of patients policy. Surgical team members actively participated only rarely during the handover but instead were often present in the PACU silently during the handover. Surgical members, particularly fellows and residents seemed unsure about their role during the handover, about what was expected, or what information they needed to provide. Findings from Saleem, Paulus, Vassiliou and Parsons (2015) suggest that the majority (77-96\%) of general surgical residents, in the American and Canadian residency program, do not receive any training on patient handover prior to starting their surgical residency. Participants in this study shared similar concerns and discussed lack of role modeling and handover training in the medical curriculum. One similarity between the study findings and Cruess, Cruess, and Steinert (2008) is the impact role modeling had on the knowledge, skills and values of trainees. 
For example, senior and staff anaesthesia providers in this study were explicit in providing the details of the handover and the information being transferred to new fellow and residents.

Anaesthesia trainees were well equipped to engage in effective handover practices once on their own. This was consistent with Crues et al., (2008) who also described modeling behaviour as an important part of education.

Understanding how behaviours and actions between the team members are connected seemed to be a challenge for the participants in this study and mutual understanding of roles and responsibilities between professionals was seen as an effective strategy to optimize communication and patient safety. Having the knowledge of specific surgical procedures or aspects of intraoperative care was an expectation of the PACU nurses. However, this knowledge is not necessarily supported by education or the PACU nurses scope of practice. At times there was also lack of understanding of roles and responsibilities in relation to the handover between the doctors and nurses. A possible explanation for this is the different perceptions among HCPs about the understanding of the information and needs of individual team members. A prospective, cross-sectional study conducting surveys about handover practices found that only $24 \%$ of participants confirmed their understanding of the information they were provided during handover (Manias et al., 2015). Interestingly, only $12 \%$ of participants said other HCPs always confirm the information and doctors were seeking more of this confirmation than other HCPs (Manias et al., 2015). There was no difference among the HCPs in requiring clarification of the information, $54 \%$ of participants believed they sometimes need to clarify the information during the handover and $8 \%$ of participants believed the information always needs to be clarified.

The participants also found that lack of clarity concerning their roles and responsibilities lead to confusion and delays in patient care. At times there were issues surrounding the medical 
and nursing responsibilities in relation to professional standards of care and requirements. Nurses felt this misunderstanding placed them in a vulnerable position, especially when they were professionally bound to clarify tasks. For example, despite the central venous line being used in the operating room throughout the procedure by the anaesthetist, according to nursing's standards of practice, PACU nurses require a written confirmation of proper placement of central line placement (CVP) to administer medication thorough the CVP.

The issue of power and hierarchy between the medical and nursing profession are well documented in the literature and misunderstanding and knowledge deficits related to professional roles are one of the barriers to successful interprofessional communication and collaboration (Hall, 2005; Rose, 2011; Strechi, 2007). Other factors that affect the doctor-nurse collaboration are the historical evolution of the profession, socialization, stereotype, and the different approaches to patient care (Strechi, 2007). A significant difference was found between the attitudes of nurses and physicians on interprofessional collaboration within the perioperative setting in a study examining the attitudes toward physician-nurse collaboration (Strechi, 2007). Years of experience had a significant influence on the attitudes toward physician-nurse collaboration. An increase in physicians' years of experience improved their attitude of team collaboration whereas nurses with more years of experience had a less positive attitude towards collaboration (Strechi, 2007). This could have been the result of the physician increased knowledge of the nursing role (Strechi, 2007).

Interprofessional collaboration is crucial for effective patient care and safety. Taking ownership of one's individual responsibility and accountability for patient care and effective leadership were observed. Overall, during the interviews participants said they felt there is a mutual respect and an inter-dependence of their roles. 


\section{Distractions and interruption}

Distractions and interruptions revealed many environmental factors affecting the handover. These factors included crowded and overcrowded space with patients, staff and equipment to noise from telephones, overhead announcements and other patients. These distractions and interruptions affected the flow of the handover process and the attention and the focus of the participants. Other issues under this category were competing responsibilities and multitasking which occurred with virtually all observed handovers. Participants often felt pressure to compete between the handover report and the care of other patients or the completion of other surgical cases. Cross covering different roles, such as in-charge and coordinating, also created problems for the staff as they had to balance between being fully focused on the handover while attending to other responsibilities during the handover. There was also increased urgency to complete the handover report quickly if it fell close to the end of the shift.

The findings under this category were similar to other published literature. A grounded theory approach used by McElroy et al. (2015) to explore clinician perceptions of handover quality, although concentrating on OR-ICU handovers, noted similarities were found. The challenges with physical obstruction encountered upon arrival to the unit was found to cause potential harm to the patient (McElroy et al., 2015). One example provided was a pulled out intravenous lines (IV) and catheters being caught on equipment during the transfer. McElroy et al. (2015) also found issues with prioritizing of tasks and activities after arrival with the postsurgical patient into the unit and conflict between competing responsibilities, such as monitor placement, documentation and handover participation. Another interesting finding in the study was, that although clinicians were frustrated by interruptions and distractions during the 
handover there was mutual understanding between the HCPs that these distractions are unavoidable in the health care environment (McElroy et al., 2015)

Interruptions were also found to be of concern in an observational descriptive study which explored factors influencing the handover and the receiver memory (Randmaa et al., 2014). The results revealed that interruptions occurred in $77 \%$ out of the 73 handovers observed and were most frequently caused by monitoring equipment signals and alarms. Furthermore, multitasking, such as monitors and infusion care or documenting in the anaesthesia chart was observed in 33\% of the 73 handovers. Randmaa et al. (2014) found a significant relationship between the handover structure (reported item sequence, duration of the handover, interruptions) and the items remembered. Additionally, in a national survey of HCPs on the subject of handovers, Van Reisen et al. (2012) found that multitasking during handovers was common and many HCPs were unaware of it happening. The results revealed that the majority of the surveyed participants $(n=494)$ and the observed participants $(n=>100)$ would prefer to have full attention of all participants during the report.

The findings, related to the category of distractions and interruptions, and those described in the literature suggest that distractions and interruptions can negatively affect the handover process. The issues described by the participants in this study related to interruptions and distractions during the handover were the inability to concentrate and the disruption of the thought process. The most common distractions were environmental noises and the most common multitasking activity was placing the vital signs monitors on while settling the patient and listening to the handover report. Interestingly, there was only two handovers observed form the 52, where the handover report happened after the patient was connected to the monitors and settled by the PACU nurse. This took 54 seconds from the time the patient arrived and allowed 
the PACU nurse to fully concentrate on the handover report. Similar findings were reported by Van Reisen et al. (2012) who found that there was only a 0.2 minute difference between simultaneous and sequential handover. This would suggest that providing time for the patient to be settled in the PACU environment before staring the handover report may not significantly increase handover duration.

Absent from the literature were studies describing the effect of distractions and interruptions during handovers and on the loss of critical information and its effect on patient safety. However, there is evidence suggesting that rapid changes in focus and attention, can cause issues with memory and information retention and therefore potentially affect patient safety (McElroy et al., 2015; Randmaa et al., 2014; Van Reisen et al., 2012). Distractions and interruptions during handovers are common and there is a need to explore initiatives directed towards distractions and interruptions

\section{Transfer of information}

The study results in the transfer of information category revealed that handovers are often incomplete with a lack of information related to expectations and outcomes. Participants described a lack of standardization as contributing to the incomplete or lack of information because the information reported during the handover depended on the individual providing the report. The information reported varied and was based on what each individual believed should be reported. Specific items were consistently underreported during the handover and the participants' suggested a lack of clarity existed about what information was to be reported and by what provider. These findings are similar to other studies exploring handovers. Inconsistencies were found in the information provided during the handover in the literature; however, there 
were differences in the type of inconsistencies between the studies (Manser et al., 2010; Milby et al., 2014; Napgal et al., 2010; Siddiqui et al., 2012). This could have been related to different expectations and inconsistencies in what information are perceived important by what HCP (Siddiqui et al., 2012). Likewise, Siddiqui et al., (2012) found there was a significant difference between what participants think should be reported and what actually is reported. Furthermore, Manias et al. (2015) found there was a significant difference between professionals in how they perceive effective handover.

Transfer of information during the observations was further influenced by two factors: 1) access to information and a general understanding of the expectations, and 2) what information is important to PACU nurses. Access to information was often dependent on the participants' presence and involvement with the handover. Surgical providers were actively participating in the handover only when the immediate postoperative care in PACU directly affected the outcome of the surgery. For example, during oral and facial surgeries, neck procedures, or cases involving flaps or particular positioning where specific instructions to post-op care needed to be given. Furthermore, OR nurses were frequently not present for the entire report, and often underreported not to repeat information they believed was already given by other team members. The literature suggests that having all team members (OR and PACU nursing, anaesthesia and surgery) present during a handover improves teamwork, information flow and transfer (Caruso et al., 2015; Craig et al., 2012; Crosson, 2015; Manser et al., 2013; Mazzocco et al. 2009; Petrovic et al., 2015; Yang \& Zhang, 2016). Findings from the literature also demonstrate that having the surgical team and the perioperative nurse participating in the handover varies from institution to institution and that having a systematic approach to handovers could improve attendance of the team members during the handover (Chen et al., 2011; Bonifacio et al., 2013; Zavalkoff et al., 
2011). This was evident for example, in Yang and Zhang's (2016) study where a standardized handover protocol significantly increased the attendance of the surgical team members improving access to surgical information during the handover. There is also evidence, guidelines and recommendations that support an active role of the OR-RN during the handover to provide vital information to optimize patient care (Crosson, 2015; Rothrocks, 2014; Napgal et al., 2013).

Some information provided during the handover was often found by the PACU nurses as irrelevant without the provision of additional context in relation to the surgical procedure. For example, reporting on a specific type of dressing, drain care or a type of a Foley catheter without providing context as to their importance can have influence over the care in the postoperative area. Another interesting finding was that over-reporting, overlap and repetition of information during the handover especially without providing additional context had the receivers of the handover not absorbing the additional information provided. There was also a lack of information being provided during the handover about expectations of required care which led to the increase of follow up phone calls and created delays with patient discharge from the PACU. Over-reporting, overlap and repeat of information and lack of information related to expectations during handovers were also found in other literature (Petrovic et al., 2015; Potestio et al., 2015; Robins \& Dai, 2015). The implementation of a handover checklist resulted in significantly less phone calls and ensured necessary information was communicated (Petrovic et al., 2015; Potestio et al., 2015; Robins \& Dai, 2015).

Personal patient information, such as family status and their location, discharge planning issues related to patient cognitive status pre-op and personal belongings were often omitted during the handover. There is limited literature on the involvement of patients or assessing patient needs during OR-PACU handover. The results in this study revealed that surgical patients 
are mostly concerned with their families and loved ones being looked after during the procedure. Relating issues about personal preferences, such as wearing glasses or having dentures available in the postoperative period was also discussed. These finding are consistent with findings from Davis et al., (2014) who explored the patient and family needs during the perioperative period. Davis et al. reported that patients wanted to have access to surgical information in the postoperative period and wanted their loved ones to be informed throughout the surgery. Access to the surgeon after the procedure was also ranked high by the patients and families. Most patients in the study were also concerned with pain and nausea management (Davis et al., 2014) Patient and family involvement during the perioperative period was also reported by Manias et al. (2016) who found that close to $45 \%$ of participants felt that family and patient involvement in the handover improved the handover considerably.

In general all participants agreed that a standardized handover process would improve the communication. However, participants voiced concerns about the form of the standardized process and suggested it be easy to follow and easy to use. Suggestions varied from creating and utilizing a simple checklist or a reminder, including the PACU nurses in the debriefing phase in the operating room, or implementing an electronic version of a handover report. Similar results in another study have suggested that there were concerns about unintended consequences of checklists or protocols due to poor design or the ability to capture the complex uniqueness of each patient (McElroy et al., 2015).

Incomplete transfer of information has been related to adverse events and targeting solutions towards developing effective process for enhancing transfer is seen as a priority (Accreditation Canada, 2012; Botti et al., 2009; Catchpole et al., 2007; CPSI, 2011). Having a discussion with the perioperative team during the handover about vital and crucial information 
such as surgical and personal information, skin assessment, positioning, anaesthesia and other concerns allows for safe and effective care management (Crosson, 2015). The results further revealed that patient involvement is critical to improving patient and family satisfaction and delivering patient-family centered care.

\section{Workflow}

Workplace culture and practices influence how tasks and activities in a department are being conducted. The effect of current practices and the available resources can directly affect patient care and safety. Staff shortages and prolonged patient flow throughout the hospital were a few of the observed examples affecting patient admission and care in the PACU. The nurse patient ratio in the PACU is one-to-one, therefore team members coming with patients from the operating room had to either wait for the nurse to become available or the nurse would have to accept and crossover two patients. Further, a hospital wide bed shortage directly affected the patient flow as there was no physical space to discharge the patients from the PACU. This caused a backlog of surgical patients and the PACU was unable to accept patients from the OR causing further delays in the OR schedule.

Patient backlog in PACU is not an uncommon phenomenon and nursing shortages, decreased patient and family satisfaction, increased costs with prolonged care, and delayed surgeries as a result of backlog have been documented in the literature (Amato-Vealey et al., 2012; Inch \& Tyne, 2012; Kuo, Borycki, Kushniruk \& Lee, 2011). Issues with flow of patients from the OR to PACU due to inefficient unit and organizational structure or resources were found to result in reduced surgical efficiency and productivity, prolonged patient stay and prolonged use of resources (Amato-Vealey et al., 2012). Amato-Vealey et al. (2012) 
implemented Six Sigma methodology to improve patient flow from the PACU to the floor units. Six Sigma methodology is based on empowering employees to recognize and reduce waste. The results showed improved patient flow and discharge from PACU and improved OR efficiency. There was also increased staff satisfaction and improved ability to accommodate surgical patients in the postoperative period (Amato-Vealey et al., 2012). Another study also suggested that the physical layout, such as bed positioning, wiring, monitor positioning needs to be addressed to improve patient flow and the environment (Palmer et al., 2013).

An interesting approach to improve patient flow and to address staff (nursing and porter) shortages due to patient transfers in PACU was taken by Inch and Tyne (2012). Inch and Tyne (2012) developed a nursing role specific to the transfer of patients from PACU to the surgical floor. This model allowed for the primary nurse to remain in the PACU and be available to accept patients from the OR without having to leave with the previous patient. The initiative led to increased patient satisfaction and improved patient flow.

Additionally, having extra personnel could have a positive effect. For example, helping with other patients during the postoperative admission and adding to the normally required oneon-one patient ratio. However, in this study, extra personnel especially at peak times, was also found to cause the space to become overcrowded.

Another key finding in this study under this category was the communication and relationships among participants. There was friendly exchanges observed among participants and participants generally knew each other by name and role. However, this was not the case during the changeover of clinical fellows and residents throughout the year. It would be helpful to start the handover with the introduction of one's name and role particularly after the rotation 
changeover. Casual and part-time staff also found it difficult to remember or know all of the perioperative HCPs.

Time constrains were also acknowledged by many participants as a barrier to effective handovers. Additionally, allowing time for questions after or during the handover could be encouraged to actively engage all participants in the handover. Addressing issues and creating meaningful strategies towards improving patient flow can positively impact the handover process in the PACU.

In summary the discussion chapter incorporated further literature to elucidate the results from this study, specifically the four main categories 1) ownership, 2) distractions and interruptions, 3) transfer of information, and 4) workflow informed by the factors and processes that affected the OR-PACU handover. The four categories included understanding individual roles and responsibilities, issues affecting the attention of the participants, communication of information during the handover and concerns related to resources and local context.

\section{Limitations}

There were limitations identified in this study. One of the methodological limitations was that data was collected by a single researcher causing potential issues related to interobserver rigor. The study was also completed in one institution only and as such the results may not be applicable to other settings. This study also did not include cardiac, thoracic, neurosurgery handovers and emergency and ICU cases.

The presence of the researcher during the observations could have affected the behaviour of the participants via the Hawthorne effect which causes participants to be on their best behaviour during observations (Loiselle et al., 2011). To minimize the Hawthorne effect the 
observations took place over a number of days, further each observation was over a lengthy period of time and the researcher informally checked with the participants to clarify meaning and interpretation of the observations by the researcher.

\section{Implications}

\section{Implications for education}

Supported by the literature, results of this study suggest there is a need to address the gaps in nursing and medical in-class and clinical curriculum specific to handovers and interprofessional communication (Cruess, 2008; McElroy et al. 2015; Paige et al., 2012; Rose, 2011; Saleem et al., 2015; Strechi, 2007). Implications for the curriculum can include specifically the information expected to be communicated during the handover and the role each

profession plays during the postoperative handover. Additional educational strategies could include the creation of opportunities for professionals to engage in interprofessional collaboration to foster understanding of individual roles and responsibilities. For example, dedicating time for interprofessional in-service and educational days and further providing healthcare professionals with opportunities to engage in simulation-based training to support enhanced teamwork and attitudes related to collaboration during a handover process (Paige et al., 2012). Continuing to acknowledge the importance of positive role modeling behaviour to practicing healthcare professionals is crucial in facilitating learner expectations (Crues et al., 2008). 


\section{Implications for policy}

Implications for policy development include the support and creation of clear local, national and international guidelines and policies related to structured handover processes for all teams involved with the surgical patient during the perioperative period. For example, formalizing guidelines and policies that would support a consistent approach to a team handover and acknowledgement of these guidelines or policies from senior leaders across all involved team member groups within the organization along with further collaboration on a national level with related institutions such as the Canadian Patient Safety Institute to support a structured process to team handover.

\section{Implications for practice}

The results of this study suggest health care professionals need to actively engage in the handover process. Perioperative healthcare professionals should be encouraged and provided with the resources to actively participate in the development of a team approach to OR-PACU handover. Creating meaningful strategies and resources such as an OR-PACU team handover tool tailored to the specific environment of practice and training of staff are essential to the successful uptake of the handover process. Working closely with senior management and related departments is needed to sustain the handover process.

One strategy to consider for implementing safer handover communication approaches in practice would be the involvement of patients and families. The input from surgical patients should be sought out after when creating initiatives towards improving surgical handovers. Patients are often under the influence of the anaesthetic during the OR-PACU handover; they are sleepy and are not actively participating or contributing during the report and as a result the 
information communicated is often only medical information. However, patients interviewed in this study demonstrated there is a place for patient and family input into information related to patient specific requests they feel should be communicated and by whom during the handover, such as level of a family involvement or personal wishes. Patient involvement with surgical handovers is an understudied area which also deserves further exploration.

Finally, a conscious effort to minimize unnecessary distractions and interruptions and further strategies to create efficient and effective workflow environment needs to be considered. These could include for example placing the monitors and settling the patient upon arrival to PACU prior to starting the handover, eliminating unnecessary conversation and phone calls and focusing on the handover and working closely with administration to address patient flow and scheduling issues.

\section{Implications for research}

The implications for future research based on this study can include further exploration of the OR-PACU handover process. Day surgery and same day admit cases were included in the observations. There was no comparison of handovers between these two groups and it would be interesting to analyze for differences in the handover duration and the nature of the handover process. There was also increased urgency to complete the handover report quickly when close to the end of the shift and further studies could compare the handover duration and information communicated between regular hours and at the end of the shift.

Exploring patient outcomes, communication practices, and the development and validation of an OR-PACU handover tool are still areas for further exploration. Research 
exploring the effect and usability of various handover improvement strategies could provide additional knowledge and perspectives.

\section{Summary}

Effective OR-PACU handovers require a structured and coordinated interprofessional effort to optimize patient care and safety. The findings of this study add to the existing literature by providing an enhanced understanding of the factors and processes informing the OR-PACU handover process. The results further reinforce the need for all team members to actively participate in the development and sustaining practice of a standardized OR-PACU handover

process. There exists opportunities to involve all members of the perioperative team in creating effective strategies to enhance team communication and the handover process. 


\section{Appendix A. Recruitment Notice Patients}

\section{RESEARCH STUDY PARTICIPANTS NEEDED}

\section{We are looking for patients who are scheduled for surgery!}

Our study is looking for patients that will be having surgery. If you would like to participate you will be asked to allow the researcher, who is also a health care professional, to observe the period when you are being transferred from the operating room to the recovery room after your surgery is completed. You may also be approached by the researcher before or after the surgery while you are in the hospital to participate in a conversation related to your transfer. Participating in this study is voluntary and will not delay or affect the care you are to receive and the researcher will not be involved in your OR-PACU transfer.

If you are scheduled to have a surgical procedure and would like to participate please contact us at phone \# $x x x-x x x-x x x x$, or email us at email address provided Please leave your name and contact information and we will get back to you to confirm your eligibility to participate in this study.

Study name: Exploring team handover: development of an Operating Room (OR)-PostAnaesthetic Care Unit (PACU) team briefing tool

Study purpose: This study will look at the factors that influence patient handover process from the operating room to the recovery room. The findings from this study will be used to create a team OR-PACU handover briefing tool

Principal investigator: Marketa Gross RN, BScN, CPN(c)

Thesis Committee: Sherry Espin, PhD, RN; Rachel Grant, RN, BScN, MN; Karen LeGrow, PhD, RN; \& Zeev Friedman, M.D

This study PI is being supported by the Canadian Patient Safety Institute (CPSI), the Faculty of Community Services (FCS) and the Daphne Cockwell School of Nursing at Ryerson University. 
Appendix B. Recruitment Notice Healthcare Professionals

\section{RESEARCH STUDY PARTICIPANTS NEEDED}

\section{We are looking for:}
- OR Nurses
- PACU Nurses
- Anaesthetists

- Surgeons

- Fellows \& Residents
- Anaesthesia Assistants

directly involved with surgical patients during OR-PACU handovers on a regular basis who would like to participate in a research study examining OR-PACU handovers.

Participation is voluntary. If you are interested please contact Marketa Gross directly, or dial \# $x x x-x x x-x x x x$, or email us at email address provided. Please leave your name and contact information and we will get back to you to confirm your eligibility to participate in this study. As a participant you will be asked to allow us to:

- Observe the OR-PACU handover process

- Engage you in informal discussion about OR-PACU handovers

You may also be asked if you are interested in participating in a focus group session lasting 45 minutes to 1 hour in length.

Study name: Exploring team handover: Development of an Operating Room (OR)PostAnaesthetic Care Unit (PACU) team briefing tool.

Primary investigator: Marketa Gross RN, BScN, CPN(c)

Thesis Committee: Sherry Espin, PhD, RN; Rachel Grant, RN, MN; Karen LeGrow, PhD, RN; \& Zeev Friedman, M.D

This study PI is being supported by the Canadian Patient Safety Institute (CPSI), Faculty of Community Services (FCS) and the Daphne Cockwell School of Nursing at Ryerson University. 
Appendix C. Consent form Patients

\section{CONSENT TO PARTICIPATE IN A RESEARCH STUDY Patients}

\section{Title}

Investigator

Co-Investigator

Sponsor

\section{Exploring team handover: development of an Operating Room (OR)-PostAnaesthetic Care Unit (PACU) team briefing tool}

\author{
Marketa Gross, RN, BScN, CPN(C) $(x x x-x x x-x x x x)$
}

Sherry Espin, $\mathrm{PhD}, R N(x x x-x x x-x x x x$ ext $x x x)$

Zeev Friedman, M.D $(x x x-x x x-x x x x)$

Rachel Grant, MN, RN $(x x x-x x x-x x x x)$

Karen LeGrow, PhD, RN $(x x x-x x x-x x x x$ ext $x x x x)$

Canadian Patient Safety Institute (CPSI) and Faculty of Community Services Ryerson University provided support for the study investigator.

\section{Introduction}

You are being asked to take part in a research study. Please read this explanation about the study and its risks and benefits before you decide if you would like to take part. You should take as much time as you need to make your decision. You should ask the study investigator or study staff to explain anything that you do not understand and make sure that all of your questions have been answered before signing this consent form. Before you make your decision, feel free to talk about this study with anyone you wish. Participation in this study is voluntary.

\section{Background and Purpose}

- You are being invited to participate in a research study looking at communication between the staff involved in the transfer/handover of patients from the operating room to the recovery room which is also called the postanaesthetic care unit (PACU). You have been approached because you are a patient undergoing an operation in the operating room department ... institution name... 
- This study will involve observation of the OR-PACU handover process from the time the surgical patient enters the recovery room (PACU) until the patient handover is completed.

\section{Study Design}

This study is a descriptive qualitative analysis of observations, document collection, and informal interviews with healthcare professionals to examine the factors that influence patient handover from the operating room to the recovery room. The findings from this study will be used to create a team OR-PACU handover briefing tool.

\section{Procedure}

- If you have agreed to participate in this study, the researcher will be observing and taking notes of the staff as they work together during the time you are transferred from the operating room to the recovery room after your surgery. We will be observing the handover process and the health care providers interactions with each other and you.

- The researcher will look at your medical records after you are transferred to the recovery room for the purpose of confirming the information communicated during the handover. The researcher will not be recording any identifiable personal or health information, and the notes will not identify you in any way. The researcher's notes will focus on the interactions between care providers and you.

- The researcher is not a member of your care team and will not be participating in your transfer and handover.

- You may also be engaged in an informal conversation/ interview by the study investigator and his/her study team while in the hospital. If this is the case the researcher may approach you while you are waiting in the Surgical Admission Waiting Room or designated area for your surgery; or then after your procedure when you are ready to be discharged in the PostAnaesthetic Care unit. If you are not being discharged home on the day of the surgery, the researcher may come and talk to you one or two days following your surgery while you are still in the hospital.

The purpose of the informal interview is for the research team to ask general questions about your ideas and thoughts related to a transfer/handover from one healthcare team to another. Informal interviews last approximately 5-10 minutes, and are not audio recorded, but the interviewer will take notes of your 
conversation. You can choose not to have notes made at any time. The informal interview will not affect or delay the care you are to receive.

\section{Reminders}

It is important to remember the following things during this study:

- No identifying information about you will be collected

- You may refuse to participate in the study, or withdraw your participation at any time

- Your decision to participate will not in any way affect your quality of care.

\section{Risks Related to Being in the Study}

We do not perceive any potential risks to participating in this study. There are no medical risks associate with this study, but being in this study may make you feel uncomfortable or anxious. If you find any of the questions during the course of the study upsetting or distressing, you may refuse to answer questions or stop the interview at any time if there is any discomfort.

\section{Benefits to Being in the Study}

You may not receive any direct benefit from being in this study. Information learned from this study may be used in the future design and implementation of a team ORPACU handover briefing tool.

\section{Voluntary Participation}

Your participation in this study is voluntary. You may decide not to be in this study, or to be in the study now and then change your mind later. You may leave the study at any time without affecting your care. You may refuse to answer any question you do not want to answer, or not answer an interview question by saying "pass".

You will have access to any new information that is learned during the study that might affect your decision to stay in the study.

\section{Confidentiality}


If you agree to join this study, the study principal investigator and his/her study team will look at your personal health information and collect only the information they need to assess the effectiveness of the handover process. Personal health information is any information that could be used to identify you and includes your:

- name,

- address,

- date of birth,

- new or existing medical records, that includes types, dates and results of medical tests or procedures.

The information that is collected for the study will be kept in a locked and secure area by the principal investigator for 7 years. The researcher will not be recording any identifiable personal or health information, and the notes will not identify you in any way. Only the study team or the people or groups listed below will be allowed to look at your records. Your participation in this study also may be recorded in your medical record at this hospital.

Representatives of the ...institution name... Research Ethics Board may look at the study records and at your personal health information to check that the information collected for the study is correct and to make sure the study followed proper laws and guidelines.

All information collected during this study, including your unidentifiable personal health information, will be kept confidential and will not be shared with anyone outside the study unless required by law. You will not be named in any reports, publications, or presentations that may come from this study.

If you decide to leave the study, the information about you that was collected before you left the study will still be used. No new information will be collected without your permission.

\section{Expenses Associated with Participating in the Study}

There are no expenses associated with participating in this study.

\section{Conflict of Interest}

The investigators conducting this study have a personal and professional interest in completing this study. Their interests should not influence your decision to participate in this study. You should not feel pressured to join this study.

\section{Questions About the Study}

If you have any questions, concerns or would like to speak to the study team for any reason, please call the primary investigators Marketa Gross at $x x x-x x x-x x x x$ or Dr. Sherry Espin at $x x x-x x x-x x x x$ etx $x x x$. 
If you have any questions about your rights as a research participant or have concerns about this study, call ...name of the ...Chair of the ...institution... Research Ethics Board (REB) or the Research Ethics office number at $x x x-x x x-x x x x$. You can also contact Lynn Lavallée, Associate Professor Chair, Human Research Ethics Board Ryerson University at 416-979-5000 $\times 4791$. The REB is a group of people who oversee the ethical conduct of research studies. These people are not part of the study team. Everything that you discuss will be kept confidential.

\section{Consent}

This study has been explained to me and any questions I had have been answered. I know that I may leave the study at any time. I agree to take part in this study.

Print Study Participant's Name

Signature

Date

(You will be given a signed copy of this consent form)

My signature means that I have explained the study to the participant named above. I have answered all questions.

$\overline{\text { Print Name of Person Obtaining ConsentSignature }} \overline{\text { Date }}$

Was the participant assisted during the consent process? $\square$ YES $\square$ NO

If YES, please check the relevant box and complete the signature space below:

$\square$ The person signing below acted as a translator for the participant during the consent process and attests that the study as set out in this form was accurately translated and has had any questions answered.

Print Name of Translator

Signature

Date 
Relationship to Participant Language

$\square$ The consent form was read to the participant. The person signing below attests that the study as set out in this form was accurately explained to, and has had any questions answered.

Print Name of Witness

Signature

Date

Relationship to Participant 
Appendix D. Consent Form Healthcare Professionals

\section{CONSENT TO PARTICIPATE IN A RESEARCH STUDY}

\section{Health Care Professionals (HCP)}

Title

Investigator

Thesis Committee

Sponsor

\section{Exploring team handover: development of an Operating Room (OR)-PostAnaesthetic Care Unit (PACU) team briefing tool}

Marketa Gross, RN, BScN, CPN(C) (xxx-xxx-xxxx)

Sherry Espin, PhD, RN ( $x x x-x x x-x x x x$ ext $x x x x)$

Zeev Friedman, M.D $(x x x-x x x-x x x x)$

Rachel Grant, MN, RN $(x x x-x x x-x x x x)$

Karen LeGrow, PhD, RN ( $x x x-x x x-x x x x$ ext $x x x x)$

Canadian Patient Safety Institute (CPSI) and Faculty of Community Services Ryerson University provided support for the study investigator.

\section{Introduction}

You are being asked to take part in a research study. This research study will fulfil the requirements of a Master Thesis in Nursing. Please read this explanation about the study and its risks and benefits before you decide if you would like to take part. You should take as much time as you need to make your decision. You should ask the study investigator or study staff to explain anything that you do not understand and make sure that all of your questions have been answered before signing this consent form. Before you make your decision, feel free to talk about this study with anyone you wish.

Participation in this study is voluntary.

\section{Background and Purpose}

- You are being invited to participate in a research study looking at communication between the staff involved in the transfer/handover of patients from the operating room $(\mathrm{OR})$ to the postanaesthetic care unit (PACU). You have been approached because you are a healthcare professional involved with OR-PACU handovers at ...institution name... on a regular basis.

- Communication between health care providers plays an important role in patient safety. The potential harmful effects of ineffective communication between health 
care members resulting in important information being missed or miscommunicated are a concern during handovers. Missed or incomplete information can result in patients not receiving the appropriate care after their surgery and in legal implications to the staff involved in patient care. We believe examining communication practices and creating a team communication/briefing tool to enhance handovers will further improve patient safety.

- This study will involve approximately 30-50 hours of observation of the OR-PACU handover process from the time the surgical patient enters the recovery room (PACU) until the patient handover is completed.

- We will be also conducting short informal interviews with healthcare professionals to gain insight into safety culture during surgical patient handoffs.

- We are also looking to further establish a small focus group representing health care providers involved with OR-PACU handovers to provide input towards creating a team communication/briefing tool to enhance OR-PACU handover process.

\section{Study Design}

This study is a descriptive qualitative analysis of observations, document collection, and informal interviews with healthcare professionals to examine the factors that influence effective communication between the teams during patient handover from the operating room to recovery room. The findings from this study will be used to create a team briefing tool that will improve the OR-PACU handover process.

\section{Procedures}

- If you agree to participate, the researchers may observe you within the context of the other health care members interaction during the surgical patient OR-PACU handoff procedure.

- You may also be asked to participate in a brief discussion and/or informal interview that will not interrupt or interfere with your regular duties. Informal interviews last approximately 5-10 minutes, and are not audio recorded, but the interviewer will take notes of your conversation. You can choose not to have notes made at any time. The purpose of an informal interview is for the research team to ask follow up questions about, or confirm or disconfirm their impressions of events they have observed in the PACU and that are related to handovers.

- You may also be asked to engage in two or three small focus group sessions lasting 45 minutes to 1 hour in length. These sessions will include representation from all parties involved with the OR-PACU handover, such as OR and PACU nursing, anaesthesia, surgery, respiratory therapy, and patients. The main objective of the focus group is to provide input towards the development of a team based OR-PACU handover tool that will enhance patient safety. The focus group sessions time and location will be set to accommodate the schedule, preferences, and convenience of the majority and well in advance. The sessions will not interfere with your scheduled working hours. If you agree, the focus group session will be recorded on an audio recording device. You may not wish to be 
audio recorded for the purpose of the focus group session. If this is the case, you may continue to participate in the study; however, the researcher will be unable to include you as a member of a focus group.

- You may be observed or engaged in informal discussion/interviews on more than one occasion over the period of next two months.

\section{Reminders}

It is important to remember the following during this study:

- No identifying information about you will be collected

- You may refuse to participate in the study, or withdraw your participation at any time

- Please call the principal investigator if you have any concerns.

\section{Risks Related to Being in the Study}

One potential risk in participating in this study is that the researchers may be required by law to surrender fieldnotes taken during a procedure in the event that legal action is taken. However, this risk is mitigated by the fact that no names or other identifying information about you is being recorded in fieldnotes.

There are no medical risks associated if you take part in this study, but being in this study may make you feel uncomfortable, anxious, or distressed. If you find any of the questions during the course of the study upsetting or distressing, you may refuse to answer questions or stop the interview at any time if there is any discomfort. Please also note that you can refuse to answer questions or stop the interview at any time for any reason.

\section{Benefits to Being in the Study}

You may not receive any direct benefit from being in this study. Information learned from this study may be used in the future design and implementation of a team ORPACU handover briefing tool.

\section{Voluntary Participation}

Your participation in this study is voluntary. You may decide not to be in this study, or to be in the study now and then change your mind later. You may leave the study at any time without any effect. You may refuse to answer any question you do not want to answer, or not answer an interview question by saying "pass".

You will have access to any new information that is learned during the study that might affect your decision to stay in the study. 


\section{Confidentiality}

If you agree to join this study, the study principal investigator and his/her study team will collect only the information they need for the study. The investigators Marketa Gross, Rachel Grant and Dr. Espin will be the only team members involved in the data collection process. No identifying information about you will be recorded in observation and/or informal interview notes.

The information that is collected for the study will be kept in a locked and secure area by the study principal investigator for 7 years.

All information collected during this study is confidential and will not be shared with anyone outside the study unless required by law. You will not be named in any reports, publications, or presentations that may come from this study.

If you decide to leave the study, the information about you that was collected before you left the study will still be used. No new information will be collected without your permission.

\section{Expenses Associated with Participating in the Study}

There are no expenses associated with participating in this study. Any informal interviews will be held at your workplace to minimize cost (transportation, time, inconvenience, etc).

\section{Conflict of Interest}

The investigators conducting this study have a personal and professional interest in completing this study. Their interests should not influence your decision to participate in this study. You should not feel pressured to join this study.

\section{Questions About the Study}

If you have any questions, concerns or would like to speak to the study team for any reason, please call the investigators Marketa Gross at $x x x-x x x-x x x x$ or Dr. Sherry Espin at $x x x-x x x-x x x x$ ext. $x x x x$

If you have any questions about your rights as a research participant or have concerns about this study, call ...name of the ...Chair of the ...institution name... Research Ethics Board (REB) or the Research Ethics office number at $x x x-x x x-x x x x$. You can also contact Lynn Lavallée, Associate Professor Chair, Human Research Ethics Board Ryerson University at $416-979-5000 \times 4791$. The REB is a group of people who oversee 
the ethical conduct of research studies. These people are not part of the study team. Everything that you discuss will be kept confidential.

\section{Consent}

This study has been explained to me and any questions I had have been answered. I know that I may leave the study at any time. I agree to take part in this study.

Print Study Participant's Name Signature

Date

(You will be given a signed copy of this consent form)

My signature means that I have explained the study to the participant named above. I have answered all questions.

$\overline{\text { Print Name of Person Obtaining ConsentSignature Date }}$

Was the participant assisted during the consent process? $\square$ YES $\square$ NO

If YES, please check the relevant box and complete the signature space below:

$\square$ The person signing below acted as a translator for the participant during the consent process and attests that the study as set out in this form was accurately translated and has had any questions answered.

$\overline{\text { Print Name of Translator }} \quad \overline{\text { Signature }}$ Date

Relationship to Participant

Language

$\square$ The consent form was read to the participant. The person signing below attests that the study as set out in this form was accurately explained to, and has had any questions answered. 
Print Name of Witness

Signature

Date

Relationship to Participant 
Appendix E. Consent Form Healthcare Professionals- Focus Group

\title{
ADDITIONAL CONSENT TO PARTICIPATE IN A RESEARCH STUDY
}

\author{
Health Care Professionals (HCP) \\ Focus Group Sessions
}

Title

Investigator

Thesis Committee

Sponsor

\begin{abstract}
Exploring team handover: development of an Operating Room (OR)-PostAnaesthetic Care Unit (PACU) team briefing tool
\end{abstract}

Marketa Gross, RN, BScN, CPN(C) (xxx-xxx-xxxx)

Sherry Espin, PhD, RN $(x x x-x x x-x x x x$ ext $x x x x)$

Zeev Friedman, M.D $(x x x-x x x-x x x x)$

Rachel Grant, MN, RN $(x x x-x x x-x x x x)$

Karen LeGrow, PhD, RN ( $x x x-x x x-x x x x$ ext $x x x x)$

Canadian Patient Safety Institute (CPSI) and Faculty of Community Services Ryerson University provided support for the study investigator.

\section{Introduction}

You are being asked to take part in a research study. This research study will fulfil the requirements of a Master Thesis in Nursing. Please read this explanation about the study and its risks and benefits before you decide if you would like to take part. You should take as much time as you need to make your decision. You should ask the study investigator or study staff to explain anything that you do not understand and make sure that all of your questions have been answered before signing this consent form. Before you make your decision, feel free to talk about this study with anyone you wish.

Participation in this study is voluntary.

\section{Background and Purpose}

- You are being invited to participate in a research study looking at communication between the staff involved in the transfer/handover of patients form the operating room (OR) to the postanaesthetic care unit (PACU). You have been approached because you are a healthcare professional involved with OR-PACU handovers at ...institution name... on a regular basis. 
- Communication between health care providers plays an important role in patient safety. The potential harmful effects of ineffective communication between health care members resulting in important information being missed or miscommunicated are a concern during handovers. Missed or incomplete information can result in patients not receiving the appropriate care after their surgery and in legal implications to the staff involved in the patient care. We believe examining communication practices and creating a team communication/briefing tool to enhance handovers will further improve patient safety.

- This study will involve approximately 30-50 hours of observation of the OR-PACU handover process from the time the surgical patient enters the recovery room (PACU) until the patient handover is completed.

- We will be also conducting short interviews with healthcare professionals to gain insight into safety culture during surgical patient handoffs.

- We are also looking to further establish a small focus group respresenting health care providers involved with OR-PACU handovers to provide input towards creating a team communication/briefing tool to enhance OR-PACU handover process.

\section{Study Design}

This study is a descriptive qualitative analysis of observations, document collection, and informal interviews with healthcare professionals to examine the factors that influence effective communication between the teams during patient handover from the operating room to recovery room. The findings from this study will be used to create a team briefing tool that will improve the OR-PACU handover process.

\section{Procedures}

- If you agree to participate you will be asked to engage in a focus group session lasting 45 to 1 hour in length. The sessions will include representation from all parties involved with the OR-PACU handover, such as OR and PACU nursing, anaesthesia, surgery, and anaesthesia assistants.

- You will be engaged in dicussions related to OR-PACU Handover and we are interested in learning more about your experience with handovers and your ideas for an effective handover process.

- The main objective of the focus group is to provide better understanding of the handover process and to provide input towards the development of a team based OR-PACU handover tool that will enhance patient safety.

- The focus group session time and location will be set to accommodate the schedule, preferences, and convenience of the majority and 6-8 weeks in advance.

- The sessions will not interfere with your scheduled working hours.

- The sessions will be recorded on an audio recording device. You may not wish to be audio recorded for the purpose of the focus group session. If this is the case, 
we thank you for you time and interest; however, the researcher will be unable to include you as a member of a focus group.

- After the session, the audiorecording will be transcribed, and any names will be replaced with a unique identifier. Any other identifying information will be removed from the transcript. The audio recording will then be destroyed. Transcriptions will be stored as an encrypted file on a password protected computer.

- You can withdraw from the study at any time by indicating to the researcher that you no longer wish to participate. You will have an opportunity to review the transcript of the focus group session prior to it being used for data analysis. You may withdraw your transcript from the study at any time by contacting the principal investigator Marketa Gross at $x x x-x x x-x x x x$. You may refuse to participate in the focus group sessions at anytime without it affecting your employment status.

\section{Reminders}

It is important to remember the following during this study:

- No identifying information about you will be collected

- You may refuse to participate in the study, or withdraw your participation at any time

- Please call the principal investigator, Marketa Gross $(x x x-x x x-x x x x)$ or Dr. Sherry Espin ( $x x x-x x x-x x x x$ ext $x x x x)$ if you have any concerns.

\section{Risks Related to Being in the Study}

- There are no medical or other risks associated if you take part in this study, but being in this study may make you feel uncomfortable, anxious, or distressed. Parts of the discussion during the focus group sessions may make you uncomfortable or upset, and you are free to decline to answer any question you do not wish to answer and/or remain quiet during the group sessions.

\section{Benefits to Being in the Study}

You may not receive any direct benefit from being in this study. Information learned from this study may be used in the future design and implementation of a team ORPACU handover briefing tool.

\section{Voluntary Participation}

Your participation in this study is voluntary. You may decide not to be in this study, or to be in the study now and then change your mind later. You may leave the study at any time without any effect. You may refuse to answer any question you do not want to answer, or not answer an interview question by saying "pass". 
You will have access to any new information that is learned during the study that might affect your decision to stay in the study.

\section{Confidentiality}

If you agree to join this study, the study principal investigator and his/her study team will collect only the information they need for the study. The investigator Marketa Gross, Rachel Grant and Dr. Espin will be the only team members involved in the data collection process related to the focus group session, however, there maybe a professional transcriber present at the sessions.

Once the focus group sessions are transcribed, the principal investigator will remove all identifying information to ensure your anonymity.

No identifying information about you will be recorded. All information associated with this study will be kept on a secure password-protected, encrypted computer at ...institution name..., accessible only by the listed study investigators. No other administrators, employees or clinicians at ...institution name... will have access to data that contains personal or identifying information in observation, focus group sessions and/or informal interview notes.

All information collected during this study is confidential and will not be shared with anyone outside the study unless required by law. However, confidentiality cannot be guarantee on behalf of other participants in the focus group, although it will be requested. You will not be named in any reports, publications, or presentations that may come from this study.

If you decide to leave the study, the information about you that was collected before you left the study will still be used. No new information will be collected without your permission.

\section{Expenses Associated with Participating in the Study}

There are no expenses associated with participating in this study. Any focus group sessions will be held at your workplace to minimize cost (transportation, time, inconvenience, etc). However, as a token of appreciation for your participation and time commitment, a $\$ 20.00$ dollar value gift card to Tim Hortons will be provided to each focus group participant at the completion of the sessions.

\section{Conflict of Interest}

The investigators conducting this study have a personal and professional interest in completing this study. Their interests should not influence your decision to participate in this study. You should not feel pressured to join this study. 


\section{Questions About the Study}

If you have any questions, concerns or would like to speak to the study team for any reason, please call the investigators Marketa Gross at $x x x-x x x-x x x x$ or Dr. Sherry Espin at $x X X-x X X-X X X X$ ext $x x x$.

If you have any questions about your rights as a research participant or have concerns about this study, call ...name of the ...Chair of the ...institution... Research Ethics Board (REB) or the Research Ethics office number at $x x x-x x x-x x x x$. You can also contact Lynn Lavallée, Associate Professor Chair, Human Research Ethics Board Ryerson University at 416-979-5000 $\times 4791$. The REB is a group of people who oversee the ethical conduct of research studies. These people are not part of the study team. Everything that you discuss will be kept confidential.

\section{Consent}

This study has been explained to me and any questions I had have been answered. I know that I may leave the study at any time. I agree to take part in this study.

Print Study Participant's Name

Signature

Date

(You will be given a signed copy of this consent form)

My signature means that I have explained the study to the participant named above. I have answered all questions.

$\overline{\text { Print Name of Person Obtaining ConsentSignature Date }}$

Was the participant assisted during the consent process? $\square$ YES $\square$ NO

If YES, please check the relevant box and complete the signature space below:

The person signing below acted as a translator for the participant during the consent process and attests that the study as set out in this form was accurately translated and has had any questions answered.

Print Name of Translator

Signature Date


Relationship to Participant Language

$\square$ The consent form was read to the participant. The person signing below attests that the study as set out in this form was accurately explained to, and has had any questions answered.

Print Name of Witness

Signature Date

Relationship to Participant 
Appendix F. Consent Form Patients- Additional Verbal

\section{Script Used to Verbally Approach and Consent Patients}

Study Title: Exploring team handover: development of an Operating Room (OR)-

PostAnaesthetic Care Unit (PACU) team briefing tool

\section{Introduction:}

The patient will be informed about the study by their health care provider and asked for permission to meet the researcher. After the patient has agreed to meet the researcher the researcher will come I and continue with:

"Hello, my name is (Marketa Gross). I am working with Dr. Sherry Espin, an associate professor and a researcher form Ryerson University and we are gathering information about patients being transferred from the operating room to the recovery area (PACU).

"I would like to observe the teamwork during the time you come out of the operating room and are being settled in the recovery room (PACU). This will include me taking notes about the transfer. There will be no personal data collected or documented in the notes and no one will be able to identify you in these notes. Do you have any questions?"

Before proceeding the researcher will ask the patient "I also need to ask you whether you are involved in any other studies or research?"

After any questions have been answered and after it was established the patient is not involved in any other study/studies that would interfere with this particular study, the researcher will continue, "In order for me observe and/or ask you questions related to our study I will need you to sign a consent form."

If the patient does not give verbal and written consent, the researcher will not engage in the observations of the handover. 


\section{Appendix G. Consent Form Healthcare Professionals- Additional Verbal}

\section{Script Used to Verbally Approach and Consent Healthcare Professionals}

Study Title: Exploring team handover: development of an Operating Room (OR)-

\section{Introduction:}

PostAnaesthetic Care Unit (PACU) team briefing tool

"Hi, my name is (Marketa Gross). I am working with Dr. Sherry Espin, an associate professor and a researcher form Ryerson University and we are gathering data related to OR-PACU handovers to be used to inform a development and implementation of a team OR-PACU handover briefing tool.

"I would like to observe the handover of the surgical patient as part of the data collection process, and I will be taking notes based on my observations. I'm not focusing on any one individual's practice in my observations, but rather, on how the team works together, and the extent to which every team member and patients are involved. All the data I'm collecting will be anonymized, and no one will be able to identify you in these notes. Do you have any questions?"

Before proceeding the researcher will ask the HCP "I also need to ask you whether you are involved in any other studies or research?"

After any questions have been answered and after it was established the HCP is not involved in any other study/studies that would interfere with this particular study, the researcher will continue, "In order for me observe and/or ask you questions related to our study I will need you to sign a consent form."

After any questions about the consent and study have been answered and the consent form was signed, the researcher will continue, "Is it ok with you if I stay and observe? If at any time you are uncomfortable with being observed, please do not hesitate to let me know and I will leave/will not observe."

If the HCP does not give verbal and written consent, the researcher does not continue with observations of the handover.

\section{Subsequent Observations:}

"Hi, do you remember me? My name is (Marketa Gross) and I was here (date), working on a study related to OR-PACU Handovers with Dr. Espin form Ryerson University".

If it is clear that the HCP remembers the researcher, she will continue, "Is it ok if I observe again?

If the HCP does not remember the researcher, the researcher will repeat the "introduction" part above. 
Appendix H. Sample Questions Patients

\begin{tabular}{|l|l|l|}
\hline \multicolumn{2}{|c|}{ Patients- Sample questions: Informal Interview } \\
\hline Time: & Place: & Type of surgery: \\
Date: & Duration: & \\
& & 0 Pre-op \\
& $\circ$ Post-op \\
\hline
\end{tabular}

The researcher may approach the patient who agreed to participate in the study and who have signed informed consent in a private and confidential manner without any disruptions in the patient care.

\section{Sample QUESTIONS:}

1. What type of information do you think is important to be transferred from one healthcare team to another healthcare team for patients who are in the hospital?

2. What type of information do you think is important to be communicated from one healthcare team to another healthcare team for patients who are coming from surgery to the recovery room?

3. Can you tell me if you see any benefits of the patient family being present during the transfer or report being given after coming from the operating room to the recovery room?

4. Can you tell me why someone may feel the patient family should not be present during the transfer or report being given after coming from the operating room to the recovery room?

At the end of the informal interview the interviewer will follow with:

"Thank you for taking the time to answer my questions. There are no names or other specific identifying information about you being recorded. The purpose of asking you these questions is for me and the research team to gain better understanding about teamwork and patient transfer from the operating room to the recovery area." 
Appendix I. Sample Questions Healthcare Professionals

\begin{tabular}{|l|l|l|}
\hline \multicolumn{3}{|c|}{ Appendix 11: HCP- Sample questions: Informal Interview } \\
\hline Time: & Place: & Role/Title of Interviewee: \\
Date: & Duration: & \\
\hline
\end{tabular}

The researcher will approach the $\mathrm{HCP}$ professionals who agreed to participate in the study and who have signed informed consent in a private and confidential manner during the work hours at times minimizing any disruptions in the regular workflow and work responsibilities.

\section{Sample QUESTIONS:}

1. What is your role in the surgical patient handover?

2. What do you think is working during handovers?

3. What do you think is not working during handovers?

4. What information do you feel is the most important to be communicated during handovers?

5. What information do you feel is the least important to be communicated during handovers?

6. Who do you thing should be involved during handovers?

7. What are the specific components you believe should be included in the handover

8. Are there any formal policies and procedures related to handovers?

9. Do you see patients being involved with handovers? For example, should they be able to decide what information is important to them to be communicated during their post-op handover?

At the end of the informal interview the interviewer will follow with:

"Thank you for taking the time to answer my questions. There are no names or other specific identifying information about you being recorded. The purpose of asking you these questions is for me and the research team to gain better understanding about handovers (and /or to follow up on a handover)." 


\section{Appendix J. Appreciation Letter}

Date

\section{Dear (name of the participant)}

I would like to thank you for your participation in our study entitled Exploring Team Handover: Development of an Operating Room (OR)-Post Anaesthetic Care Unit (PACU) Team Briefing Tool.

The purpose of the study was to explore the communication practices and factors influencing the OR-PACU handover process during the transfer/handover of patients from the operating room (OR) to the postanaesthetic care unit (PACU).

The data collected during the observations, informal interviews, and during the focus group sessions will contribute to a better understanding of the OR-PACU handover process and will be used towards the development of a team OR-PACU team handover briefing tool to enhance handovers and further improve patient safety.

Please remember that any information and data pertaining to you as an individual participant will be kept confidential. The results of this study will be shared with the research community through conferences, presentations, and journal articles. If you are interested in receiving more information regarding the results of this study please provide your email address and we will send the summary of the results when available, anticipated by ...date inserted .... In the meantime, if you have any further questions about the study, please do not hesitate to contact me by email or telephone as noted below. The study was approved by the Research Ethics Board of ... institution name... and Ryerson University. Should you have any comments or concerns resulting from your participation in this study you can also contact ...name of the... Chair of the ... institution name... Research Ethics Board (REB) or the Research Ethics Office at 416-5864875. You can also contact Lynn Lavallée, Associate Professor Chair, Human Research Ethics Board Ryerson University at 416-979-5000 x 4791.

Sincerely,

Marketa Gross

Email: ...email provided...

Telephone: $x x x-x x x-x x x x$ 


\section{Appendix K. Appreciation Letter- Focus Group Participants}

Date

Dear participant,

I would like to thank you for your participation in the focus group session which is a part of our study titled Exploring Team Handover: Development of an Operating Room (OR)-Post Anaesthetic Care Unit (PACU) Team Briefing Tool.

The purpose of the session was to discuss the communication practices and factors that influence the OR-PACU handover process and to create a draft of a team handover briefing tool/guide to enhance OR-PACU handovers and further improve patient safety.

Please remember that any information and data pertaining to you as an individual participant will be kept confidential. I may contact you via email or in person to follow up on the draft as discussed during the focus group session. You are not required to engage in any follow up conversations if you chose not to do so.

Further, the results of this study will be shared with the research community through conferences, presentations, and journal articles. If you are interested in receiving more information regarding the final results of this study please provide your email address and we will send the summary of the results when available, anticipated by ...date inserted.... We will also provide staff briefing sessions upon the study completion. In the meantime, if you have any further questions about the study, please do not hesitate to contact me by email or telephone as noted below. The study was approved by the Research Ethics Board of ...institution name... and Ryerson University. Should you have any comments or concerns resulting from your participation in this study you can also ...name of the ... Chair of the ...institution name... Research Ethics Board (REB) or the Research Ethics Office at $x x x-x x x-x x x x$. You can also contact Lynn Lavallée, Associate Professor Chair, Human Research Ethics Board Ryerson University at 416-979-5000 x 4791.

Enclosed is a gift certificate in the value of $\$ 20.00$ as a token of appreciation for you time and participation.

Sincerely,

Marketa

Email: ...email provided... or ....email provided....

Telephone: $x x x-x x x-x x x x$ 


\section{References}

Accreditation Canada (2016). Required Organizational Practices: Handbook 2016. Retrieved from https://accreditation.ca/sites/default/files/rop-handbook-2016-en.pdf

Agarwal, H. S., Saville, B. R., Slayton, J. M., Donahue, B. S., Daves, S., Christian, K. G., . . Harris, Z. L. (2012). Standardized postoperative handover process improves outcomes in the intensive care unit: A model for operational sustainability and improved team performance. Critical Care Medicine, 40(7), 2109-2115.

doi:10.1097/CCM.0b013e3182514bab

Amato-Vealey, E. J., Barba, M. P., \& Vealey, R. J. (2008). Hand-off communication: A requisite for perioperative patient safety. AORN Journal, 88(5), 763-774.

doi:10.1016/j.aorn.2008.07.022

Arenas, A., Tabaac, B. J., Fastovets, G., \& Patil, V. (2014). Undivided attention improves postoperative anesthesia handover recall. Journal of Advances in Medical Education and Practice, 5, 215-220. doi:10.2147/amep.s65361

Baker, G. R., Norton, P. G., Flintoft, V., Blais, R., Brown, A., Cox, J, ...\& Tamblin, R. (2004). The Canadian adverse events study: The incidence of adverse events among hospital patients in Canada. Canadian Medical Association Journal, 170(11), 1678-1686. doi:10.1053/cmaj.1040498

Boat, A. C., \& Spaeth, J. P. (2013). Handoff checklists improve the reliability of patient handoffs in the operating room and postanesthesia care unit. Pediatric Anesthesia, 23(7), 647-654. doi: 10.1111/pan.12199 
Bonifacio, A. S., Segall, N., Barbeito, A., Taekman, J., Schroeder, R., \& Mark, J. B. (2013).

Handovers from the OR to the ICU. International Anesthesiology Clinics, 51(1), 43-61. doi:10.1097/AIA.0b013e31826f2b0e

Boreham, N. (2004). A theory of collective competence: Challenging the neo-liberal individualisation of performance at work. British Journal of Educational Studies, 52(1), 5-17. Retrieved from http://www.tandfonline.com/toc/rbje20/current

Botti, M., Bucknall, T., Cameron, P., Johnstone, M. J., Redley, B., Evans, S., \& Jeffcott, S. (2009). Examining communication and team performance during clinical handover in a complex environment: The private sector post-anaesthetic care unit. Medical Journal of Australia, 190(11 Suppl), S157-160 151p.

Bourdon, L. (2015). Standardizing the OR to PACU patient hand over. AORN Journal, 101(2), 10-12. doi: http://dx.doi.org/10.1016/S0001-2092(14)01421-5

Canadian Patient Safety Institute. (2011). The Economics of Patient Safety in Acute Care: Technical Report. Retrieved form: http://www.patientsafetyinstitute.ca/en/toolsResources/Research/commissionedRe search/EconomicsofPatientSafety/Documents/Economics\%20of\%20Patient $\% 20 \mathrm{~S}$ afety\%20-\%20Acute\%20Care\%20-\%20Final\%20Report.pdf

Canadian Institute of Health Research, Natural Sciences and Engineering Research Council of Canada, \& Social Sciences and Humanities Research Council of Canada. (2014). The Tri-Council Policy Statement: Ethical Conduct for Research Involving Human Subjects. Retrieved from http://www.pre.ethics.gc.ca/pdf/eng/tcps2-2014/TCPS_2_FINAL_Web.pdf 
Canadian Medical Protective Association. (2013) Action for Safer Medical Care: Patient Handovers. Retrieved from https://www.cmpaacpm.ca/documents/10179/300031190/patient_handovers-e.pdf

Caruso, T. J., Marquez, J. L., Wu, D. S., Shaffer, J. A., Balise, R. R., Groom, M., . . Sharek, P. J. (2015). Implementation of a standardized postanesthesia care handoff increases information transfer without increasing handoff duration. The Joint Commission Journal on Quality and Patient Safety, 41(1), 35-42. Retrieved from http://www.jcrinc.com/thejoint-commission-journal-on-quality-and-patient-safety/

Chen, J. G., Wright, M. C., Smith, P. B., Jaggers, J., \& Mistry, K. P. (2011). Adaptation of a postoperative handoff communication process for children with heart disease: A quantitative study. Americam Journal of Medical Quality, 26(5), 380-386. doi: $10.1177 / 1062860610394342$

Coleman, K., Redley, B., Wood, B., Bucknall, T., \& Botti, M. (2015). Interprofessional interactions influence nurses' adoption of handover improvement. ACORN: The Journal of Perioperative Nursing in Australia, 28(1), 10-14. https://www.acorn.org.au/journal/

Crosson, J. A. (2015). Keeping patients safe: The importance of collaboration. AORN Journal, 101, 279-281 273p. doi:10.1016/j.aorn.2014.11.006

Craig, R., Moxey, L., Young, D., Spenceley, N. S., \& Davidson, M. G. (2012). Strengthening handover communication in pediatric cardiac intensive care. Paediatric Anaesthesia, 22(4), 393-399. doi:10.1111/j.1460-9592.2011.03758.x

Creswell, J. W. (2013). Qualitative inquiry and research design: Choosing among five approaches. $\left(3^{\text {rd }}\right.$ ed.). Thousand Oaks, CA: Sage 
Cruess, S. R., Cruess, R. L., \& Steinert, Y. (2008). Role modelling-making the most of a powerful teaching strategy. BMJ : British Medical Journal, 336(7646), 718-721. doi:10.1136/bmj.39503.757847.BE

Davis, Y., Perham, M., Hurd, A. M., Jagersky, R., Gorman, W. J., Lynch-Carlson, D., \& Senseney, D. (2014). Patient and family member needs during the perioperative period. Journal of PeriAnesthesia Nursing, 29(2), 119-128. doi: http://dx.doi.org/10.1016/j.jopan.2013.05.013

Dearden, K., \& Hewitt, C. (2004). Benchmarking in post-anaesthetic care. Nursing Standard, 18(42), 42-4. Retrieved from http://www.nursing-standardjournal.co.uk/

Elliott, R., \& Timulak, L. (2005). Descriptive and interpretive approaches to qualitative research. In A Handbook of Research Methods for Clinical and Health Psychology. New York, NY: Oxford University Press.

Elo, S., \& Kyngas, H. (2008). The qualitative content analysis process. Journal of Advanced Nursing, 62(1), 107-115. doi:10.1111/j.1365-2648.2007.04569.x

Fabila, T. S., Hee, H. I., Sultana, R., Assam, P. N., Kiew, A., \& Chan, Y. H. (2016). Improving postoperative handover from anaesthetists to non-anaesthetists in a children's intensive care unit: The receiver's perception. Singapore Medical Journal, 57(5), 242-253. doi:10.11622/smedj.2016090

Funk, E., Taicher, B., Thompson, J., Iannello, K., Morgan, B., \& Hawks, S. (2016). Structured handover in the pediatric postanesthesia care unit. Journal of PeriAnesthesia Nursing, 31(1), 63-72. doi:10.1016/j.jopan.2014.07.015 
Graneheim, U. H., \& Lundman, B. (2004). Qualitative content analysis in nursing research: Concepts, procedures and measures to achieve trustworthiness. Nurse Education Today, 24(2), 105-112. doi:http://dx.doi.org/10.1016/j.nedt.2003.10.001

Grover, A., \& Duggan, E. (2013). Chinese whispers in the post anaesthesia care unit (PACU). Irish Medical Journal, 106(8), 241-243. Retrieved from http://imj.ie/

Gurses, A. P., Kim, G., Martinez, E. A., Marsteller, J., Bauer, L., Lubomski, L. H., Provonost, P. J., \& Thompson, D. (2012). Identifying and categorizing patient safety hazards in cardiovascular operating rooms using an interdisciplinary approach: A multisite study. BMJ Quality and Safety, 21(10), 810-818. Retrieved from http://qualitysafety.bmj.com/ Hall, P. (2005). Interprofessional teamwork: Professional cultures as barriers. Journal of Interprofessional Care, Supplement (1).188 - 196. doi: 10.1080/13561820500081745

Halverson, A. L., Casey, J. T., Anderson, J., Anderson, K., Park, C., Rademaker, A. W., \& Moorman, D. (2010). Communication failure in the operating room. Surgery, 149(3). 305-310. doi:10.1016/j.surg.2010.07.051

Healey, A. N., Undre, S., Sevdalis, N., Koutantji, M., \& Vincent, C. A. (2006). The complexity of measuring interprofessional teamwork in the operating theatre. Journal of Interprofessional Care, 20(5), 485-495. doi:10.1080/13561820600937473

Hodges, B. D., Lingard, L. (2012). The question of competence: Reconstructing medical education in the twenty-first century. ILR press. Corneal University Press, Ithaca and London.

Hsieh, H-F., \& Shannon, S. E. (2005). Three approaches to qualitative content analysis. Qualitative Health Research, 15(9), 1277-1288. doi: 10.1177/1049732305276687 
Huang, L., Kim, R., \& Berry, W. (2013). Creating culture of safety by using checklist. AORN Journal, 97(3). 365-179. doi:http://dx.doi.org/10.1016/j.aorn.2012.12.019

Iacono, M. V. (2009). Handoff Communication: Opportunities for Improvement. Journal of PeriAnesthesia Nursing, 24(5), 324-326. doi:10.1016/j.jopan.2009.08.003

Inch, J., \& Tyne, S. (2013). Innovation the EOC way the appointment of a transfer link nurse: Is it the answer to a busy PACU's prayers? British Journal of Anaesthetic \& Recovery Nursing, 14(1-2). 26-29. doi:10.1017/S1742645613000077

Joint Commission Center for Transforming Healthcare, (2014). Improving Transitions of Care: Hand-off Communications. Retrieved from http://www.centerfortransforminghealthcare.org/assets/4/6/handoff_comm_storyboard.pd $\underline{\mathrm{f}}$

Joint Commission on the Accreditation of Healthcare Organizations, (2015). Sentinel Event Data Root Causes by Event Type 2004 - 2Q 2014. Retrieved from http://www.jointcommission.org/assets/1/18/Root_Causes_by_Event_Type_20042Q 2014.pdf

Joy, B. F., Elliott, E., Hardy, C., Sullivan, C., Backer, C. L., \& Kane, J. M. (2011). Standardized multidisciplinary protocol improves handover of cardiac surgery patients to the intensive care unit. Pediatric Critical Care Medicine, 12(3), 304308. doi: 10.1097/PCC.0b013e3181fe25a1

Kim, S. W., Maturo, S., Dwyer, D., Monash, B., Yager, P. H., Zanger, K., \& Hartnick, C. J. (2012). Interdisciplinary development and implementation of communication checklist for postoperative management of pediatric airway patients. 
Otolaryngology - Head \& Neck Surgery, 146(1), 129-134. doi:

$10.1177 / 0194599811421745$

Kitney, P., Tam, R., Bennett, P., Buttigieg, D., Bramley, D., \& Wei, W. (2016).

Handover between anaesthetists and post-anaesthetic care unit nursing staff using ISBAR principles: A quality improvement study. ACORN: The Journal of Perioperative Nursing in Australia, 29(1), 30-35. Retrieved from https://www.acorn.org.au/journal/

Kitto, S., Chesters, J., \& Grbich, C. (2008). Quality in qualitative research. Medical Journal of Australia 188(4), 243-246. Retrieved from https://www.mja.com.au/

Krippendorff, K. (2004). Content analysis: An introduction to its methodology. (2nd ed.). Thousand Oaks, CA: Sage.

Kuo, A., Borycki, E., Kushniruk, A., \& Lee, T.-S. (2011). A healthcare lean Six Sigma system for postanesthesia care unit workflow improvement. Quality Management in Healthcare, 20(1), 4-14. doi: 10.1097/QMH.0b013e3182033791

Lingard, L., Regehr, G., Espin, S., \& Whyte, S. (2006). A theory based instrument to evaluate team communication in the operating room: Balancing measurement authenticity and reliability. BMJ Quality \& Safety, 15(6), 422-426. Retrieved from http://qualitysafety.bmj.com/

Lingard, L., Reznick, R., Espin, S., Regher, L., \& DeVito, I. (2002). Team communication in the operating room: Talk, patterns, sites of tension, and implications for novices. Academic Medicine, 77(3), 232-237. Retrieved from http://journals.lww.com/academicmedicine/pages/default.aspx 
Lingard, L., Regehr, L., Orser, B., Reznick, R., Baker, G. R., Doran, D., ... \& Whyte, S. (2008). Evaluation of a preoperative checklist and team briefing among surgeons, nurses, and anesthesiologists to reduce failures in communication. Archives of Surgery, 143(1), 12-17. doi: 10.1001/archsurg.2007.21

Loiselle, C. G., Profetto-McGrath, J. Polit, P., D., \& Tatano Beck C. (2011). Canadian Essentials of Nursing Research ( $3^{\text {rd }}$ ed.). Philadelphia: Lippincott Williams \& Wilkins

Low, D. K., Reed, M. A., Geiduschek, J. M., \& Martin, L. D. (2013). Striving for a zeroerror patient surgical journey through adoption of aviation-style challenge and response flow checklists: A quality improvement project. Paediatric Anaesthesia, 23(7), 571-578. doi:10.1111/pan.12121

Manias, E., Geddes, F., Watson, B., Jones, D., \& Della, P. (2016). Perspectives of clinical handover process: A multi-site survey across different health professionals. Journal of Clinical Nursing, (1-2)25, 80-91. doi:

10.1111/jocn. 12986

Manser, T., Foster, S., Gisin, S., Jeackel, D., \& Ummenhofer, W. (2010). Assessing the quality of patient handoffs at care transition. Quality and Safety in Health Care, (19)6e44. doi:10.1136/qshc.2009.038430

Manser, T., Foster, S., Flin, R., \& Patey, R. (2013). Team communication during patient handover from the operating room: more than facts and figures. Human Factors and Ergonomics Society, 55(1), 138-156. doi:10.1177/0018720812451594

Mazzocco, K., Petitti, D. B., Fong, K. T., Bonacum, D., Brookey, J., Graham, S., . . Thomas, E. J. (2009). Surgical team behaviors and patient outcomes. The 
American Journal of Surgery, 197(5), 678-685.

doi:10.1016/j.amjsurg.2008.03.002

McElroy, L. M., Collins, K. M., Koller, F. L., Khorzad, R., Abecassis, M. M., Holl, J. L., \& Ladner, D. P. (2015). Operating room to intensive care unit handoffs and the risks of patient harm. Surgery, 158(3), 588-594. doi:10.1016/j.surg.2015.03.061

Milby A., Böhmer A., Gerbershagen M. U., Joppich R., \& Wappler F. (2014). Quality of post-operative patient handover in the post-anaesthesia care unit: A prospective analysis. Acta Anesthesiologica Scandinavica, 58(2), 192-197. doi:

10.1111/aas.12249.

Mistry, K. P., Jaggers, J., Lodge, A. J., Alton, M., Mericle, J. M., Frush, K. S., \& Meliones, J. N. (2008). Using Six Sigma ${ }^{\circledR}$ methodology to improve handoff communication in high-risk patients. In: Henriksen, K, Battles, J. B, Keyes, M. A, et al., (Ed.). Advances in Patient Safety: New Directions and Alternative Approaches, Performance and Tools, 3. Rockville (MD): Agency for Healthcare Research and Quality. Retrieved from http://www.ahrq.gov/sites/default/files/wysiwyg/professionals/education/curriculumtools/teamstepps/instructor/fundamentals/module3/ebcommunication.pdf

Moorhouse, A., Yeo, M., \& Rodney, P. (2010). Autonomy. In Yeo, M., Moorehouse, A., Khan, P., \& Rodney, P. (Eds.). Concepts and cases in nursing ethics (3rd ed.). (pp. 143-206). Toronto, ON, Canada: Broadview Press.

Nagpal, K., Abboudi, M., Fischler,L., Schmidt, T., Vats, A., Manchanda, Ch., ... \& Moorthy, K. (2011). Evaluation of postoperative handover using a tool to assess information transfer and teamwork. Annals of Surgery, 253(4), 831-837. doi: 10.1097/SLA.0b013e318211d849. 
Nagpal, K., Abboudi, M., Manchanda Ch., Vats, A., Sevdalis, N., Bicknell, C., Vincent, Ch., \& Moorthy, K. (2013). Improving postoperative handover: A prospective observational study. The American Journal of Surgery, 206(4). 494-501. doi: 10.1016/j.amjsurg.2013.03.005.

Nagpal, K., Arora, S., Abboudi, M., Vats, A., Wong, H. W., Manchanda, C., . . Moorthy, K. (2010a). Postoperative handover: problems, pitfalls, and prevention of error. Annals of Surgery, 252(1), 171-176. doi: 10.1097/SLA.0b013e3181dc3656

Nagpal, K., Vats, A., Ahmed, K., Vincent, C., \& Moorthy, K. (2010b). An evaluation of information transfer through the continuum of surgical care: A feasibility study. Annals of Surgery, 252(2), 402-407. doi:10.1097/SLA.0b013e3181e986df

Nagpal, K., Vats, A., Ahmed, K., Smith, A. B., Sevdalis, N., Jonannsson, H., . . . Moorthy, K. (2010). A systematic quantitative assessment of risks associated with poor communication in surgical care. Archives of Surgery, 145(6), 582-588. doi:10.1001/archsurg.2010.105

Nagpal, K., Abboudi, M., Fischler, L., Schmidt, T., Vats, A., Manchanda, C., . . . Moorthy, K. (2011). Evaluation of postoperative handover using a tool to assess information transfer and teamwork. Annals of Surgery, 253(4), 831-837. doi:10.1097/SLA.0b013e318211d849

Nasarawanji, M.F., Badir, A., \& Gurses, A. P. (2016). Standardizing handoff communication: Content analysis of 27 handoff mnemonics. Journal of Nursing Care Quality, 31(3), 238244. Retrieved from http://journals.lww.com/jncqjournal/pages/default.aspx 
Neergaard, M. A., Olesen, F., Andersen, R. S., \& Sondergaard, J. (2009). Qualitative description - The poor cousin of health research? BMC Medical Research Methodology, 9(52). doi:10.1186/1471-2288-9-52

O’Reilly, M., \& Parker, N. (2012). Unsatisfactory Saturation: A critical exploration of the notion of saturated sample sizes in qualitative research. Qualitative Research, 13(2), 190-197. doi: $10.1177 / 1468794112446106$

Ortlipp, M. (2008). Keeping and using reflective journals in the qualitative research process. The Qualitative Report, 13(4). 695-705. http://www.nova.edu/ssss/QR/QR13-4/

Paige, J. T., Garbee, D. D., Kozmenko, V., Qinzhao, Y., Kozmenko, L., Yang, T., Bonanno, L., Swartz, W. (2014). Getting a head start: High-fidelity, simulation-based operating room team training of interprofessional students. Journal of the American College of Surgeons, 218(1), 140-149. doi:http://dx.doi.org/10.1016/j.jamcollsurg.2013.09.006

Palmer, G., Abernathy, J. H., Swinton, G., Allison, D., Greenstein, J., Shappell, S., . . Reeves, S. T. (2013). Realizing improved patient care through human-centered operating room design: A human factors methodology for observing flow disruptions in the cardiothoracic operating room. Anesthesiology, 119(5), 1066-1077. doi:10.1097/ALN.0b013e31829f68cf

Piekarski, F., Kaufmann, J., Laschat, M., Böhmer, A., Engelhardt, T., \& Wappler, F. (2015). Quality of handover in a pediatric postanesthesia care unit. Pediatric Anesthesia, 25(7), 746-752. doi:10.1111/pan.12646

Petrovic, M. A., Aboumatar, H., Scholl, A. T., Gill, R. S., Krenzischek, D. A., Camp, M. S., . . Martinez, E. A. (2015). The perioperative handoff protocol: Evaluating impacts on 
handoff defects and provider satisfaction in adult perianesthesia care units. Journal of Clinical Anesthesia, 27(2), 111-119. doi:10.1016/j.jclinane.2014.09.007

Petrovic, M. A., Aboumatar, H., Baumgartner, W. A., Ulatowski, J., A., Moyer, J., Chang, T. Y., ... \& Martinez, E. A. (2012). Implementation of perioperative protocol to guide operating room-to-intensive care unit patient handoffs. Journal of Cardiothoracic and Vascular Anaesthesia, 26(1), 11-16. doi:10.1053/j.jvca.2011.07.009

Potestio, C., Mottla, J., Kelley E., \& DeGroot, K. (2015). Improving post anesthesia care unit (PACU) handoff by implementing a succinct checklist. APSF Newsletter, 30(1). 13-15. Retrieved from http://www.apsf.org/newsletters

Pucher, P. H., Johnston, M. J., Aggarwal, R., Arora, S., \& Darzi, A. (2015). Effectiveness of interventions to improve patient handover in surgery: A systematic review. Surgery, 158(1), 85-95. doi:10.1016/j.surg.2015.02.017

Radmaa, M., Martensson, G., Swenne, C. L., \& Engrstrom, M. (2105). An observation study of postoperative handover $\mathrm{n}$ anaesthesia clinic: The content of verbal information and factors influencing receiver memory. Journal of Perianaesthesia Nursing, 30(2), 105-

115. doi: $10.1016 /$ j.jopan.2014.01.012

Randmaa, M., Swenne, C., Martensson, G., Hogberg, H., \& Engstrom, M. (2016). Implementing situation-background-assessment-recommendation in an anaesthetic clinic and subsequent information retention among receivers: A prospective interventional study of postoperative handovers. European Journal of Anaesthesiology, 33(3), 172-178. doi:10.1097/EJA.0000000000000335 
Robins, H.-M., \& Dai, F. (2015). Handoffs in the Postoperative Anesthesia Care Unit: Use of a Checklist for Transfer of Care. AANA Journal, 83(4), 264-268. Retrieved from http://www.aana.com/newsandjournal/Pages/aanajournalonline.aspx

Rose, L., (2011). Interprofessional collaboration in the ICU: how to define? Nursing in Critical Care, 16(1). 5-10. doi: 10.1111/j.1478-5153.2010.00398.x.

Rothrock, J. (2014). Alexander's care of the surgical patient. (15th ed). Toronto: CV. Mosby Ryan, S., O’Riordan, J. M., Tierney, S., Conlon, K. C., \& Ridgway, P. F. (2011). Impact of a new electronic handover system in surgery. International Journal of Surgery, 9(3), 217220. doi:10.1016/j.ijsu.2010.11.012

Saleem, A. M., Paulus, J. K., Vassiliou, M. C., \& Parsons, S. K. (2015). Initial assessment of patient handoff in accredited general surgery residency programs in the United States and Canada: A cross-sectional survey. Canadian Journal of Surgery, 58(4), 269-277.

Retrieved from http://canjsurg.ca/

Salzwedel, C., Hans-ürgen, B., Kühnelt, I., Appel, D., Haupt, O., Maisch, S., \& Schmidt, N. G. (2013). The effect of a checklist on the quality of post-anaesthesia patient handover: A randomized controlled trial. International Journal for Quality in Health Care. 25(2). 176181. doi:http://dx.doi.org/10.1093/intqhc/mzt009

Sandelowski, M. (1993). The problem of rigor or rigor mortis: The problem of rigor in qualitative research revisited. Advances in Nursing Science, 16(2), 1-8. Retrieved from http://journals.lww.com/advancesinnursingscience/Pages/default.aspx

Sandelowski, M. (2000). Whatever happened to qualitative description? Research in Nursing and Health, 23(4), 334-340. Retrieved from http://onlinelibrary.wiley.com/journal/10.1002/(ISSN)1098-240X 
Sandelowski, M. (2010). What's in a name? Qualitative description revisited. Research in Nursing and Health, 33(1), 77-84. doi:10.1002/nur.20362

Sandlin, D. (2007). Improving patient safety by implementing a standardized and consistent approach to hand-off communication. Journal of PeriAnesthesia Nursing, 22(4), 289-292. Retrieved from http://www.jopan.org/

Segall, N., Bonifacio, A., Schroeder, R. A., Barbeito, A., Rogers, D., ... \& Mark, J. B. (2012). Can we make postoperative patient handovers safer? A systematic review of the literature. Anesthesia \& Analgesia, 115(1), 102-115. doi:

10.1213/ANE.0b013e318253af4b

Sharit, J., McCane, L., Thevenin, D. M., \& Barach, P. (2008). Examining links between sign-out reporting during shift changeovers and patient management risks. Risk Analysis, 28, 969981.

Siddiqui, N., Arzola, C., Iqbal, M., Sritharan, K., Guerina, L., Chung, F., \& Friedman, Z. (2012). Deficits in information transfer between anaesthesiologist and postanaesthesia care unit staff: An analysis of patient handover. European Journal of Anaesthesiology, 29(9), 438-445. doi:10.1097/EJA.0b013e3283543e43

Smith, J., Bekker, H., \& Cheater, F. (2011). Theoretical versus pragmatic design in qualitative research. Nurse Researcher, 18(2), 39-51. Retrieved from http://journals.rcni.com/journal/nr

Smith, B., \& Jones, C. (2009). Perioperative communication. The Journal of Perioperative Practice, 19(8), 248-253. Retrieved from http://www.afpp.org.uk/books-journals/Journal-of-Perioperative-Practice 
Smith, A. F., \& Mishra, K. (2010). Interaction between anaesthetists, their patients, and the anaesthesia team. British Journal of Anaesthesia, 105(1), 60-68. doi:10.1093/bja/aeq132

Smith, A. F., Pope, C., Goodwin, D., \& Mort, M. (2008). Interprofessional handover and patient safety in anaesthesia: observational study of handovers in the recovery room. BJA: The British Journal of Anaesthesia, 101, 332-337 336p.

Strechi, S. L. (2007). Perceptions that affect physician-nurse collaboration in the perioperative setting. AORN Journal, 86(1), 45-57. Retrieved from http://www.aornjournal.org/

Streubert, H. J., \& Carpenter, D. R. (2011). Qualitative research in nursing: Advancing the humanistic imperative $\left(5^{\text {th }}\right.$ ed.). Philadelphia: PA, Lippincott, Williams \& Wilkins.

Urbach, D. R., Govindarajan, A., Saskin, R., Wilton, A. S., \& Baxter, N. N. (2014). Introduction of surgical safety checklists in Ontario, Canada. New England Journal of Medicine, 370(11), 1029-1038. doi:10.1056/NEJMsa1308261

Van Rensen, E., Thieme Groen, E., Numan, S., Smit, M., Cremer, O., Tates, K., \& Kalkman, C. (2012). Multitasking During Patient Handover in the Recovery Room. Survey of Anesthesthesia \& Analgesia, 115(5), 1183-1187. doi: 10.1213/ANE.0b013e31826996a2

Vaismoradi, M., Turunen, H., \& Bondas, T. (2013). Content analysis and thematic analysis: Implications for conducting a qualitative descriptive study. Nursing Health Science, 15(3), 398-405. doi:10.1111/nhs.12048

Vergales, J., Addison, N., Vendittelli, A., Nicholson, E., Carver, D. J., Stemland, C., . . Gangemi, J. (2015). Face-to-face handoff: Improving transfer to the pediatric intensive care unit after cardiac surgery. American Journal of Medical Quality, 30(2), 119-125. doi:10.1177/1062860613518419 
Weinger, M. B., Slagle, J. M., Kuntz, A. H., Schildcrout, J. S., Banerjee, A., Mercaldo, N. D., . . France, D. J. (2015). A multimodal intervention improves postanesthesia care unit handovers. Anesthesia \& Analgesia, 121(4), 957-971. doi:10.1213/ane.0000000000000670

Wheeler, K. (2015). Effective handoff communication. Nursing Critical Care, 10(6), 1315. doi: 10.1097/01.CCN.0000472849.85679.c4

Wilson, V. (2011). Research methods: Content analysis. Evidence based library and information practice, 6(4). 177-179. doi: http://dx.doi.org/10.18438/B86P6S

World Health Organization. (2009). WHO Guidelines for Safe Surgery 2009: Safe Surgery Saves Lives. Retrieved from http://apps.who.int/iris/bitstream/10665/44185/1/9789241598552_eng.pdf

Yang, J.-G., \& Zhang, J. (2016). Improving the postoperative handover process in the intensive care unit of a tertiary teaching hospital. Journal of Clinical Nursing, 25(7-8), 887-1172, doi:10.1111/jocn.13115

Yeo, M., Moorhouse, A., \& Krahn, I. (2010). Confidentiality. InYeo, M., Moorehouse, A., Khan, P., \& Rodney, P. (Eds.). Concepts and cases in nursing ethics $\left(3^{\mathrm{Rd}} \mathrm{ed}.\right)$. (pp.245-292). Toronto, ON, Canada: Broadview Press

Yeo, M., Moorehouse, A., Khan, P., \& Rodney, P. (Eds.). (2010). Concepts and cases in nursing ethics $\left(3^{\text {rd }}\right.$ ed.). Toronto, ON, Canada: Broadview Press.

Zavalkoff, S. R., Razack, S. I., Lavoie, J. M., \& Dancea, A. B. (2011). Handover after pediatric heart surgery: A simple tool improves information exchange. Pediatric Critical Care Medicine, 12(3), 309-313. doi: 10.1097/PCC.0b013e3181fe27b6. 
